\title{
UNIVERSAL COVERINGS OF THE ORTHOGONAL GROUPS
}

\author{
V. V. Varlamov \\ Department of Mathematics, Siberia State University of Industry, \\ Kirova 42, Novokuznetsk 654007, Russia
}

\begin{abstract}
Universal coverings of the orthogonal groups and their extensions are studied in terms of Clifford-Lipschitz groups. An algebraic description of basic discrete symmetries (space inversion $P$, time reversal $T$, charge conjugation $C$ and their combinations $P T, C P, C T, C P T)$ is given. Discrete subgroups $\{1, P, T, P T\}$ of orthogonal groups of multidimensional spaces over the fields of real and complex numbers are considered in terms of fundamental automorphisms of Clifford algebras. The fundamental automorphisms form a finite group of order 4 . The charge conjugation is represented by a complex conjugation pseudoautomorphism of the Clifford algebra. Such a description allows one to extend the automorphism group. It is shown that an extended automorphism group (CPT-group) forms a finite group of order 8 . The group structure and isomorphisms between the extended automorphism groups and finite groups are studied in detail. It is proved that there exist 64 different realizations of $C P T$-group. An extension of universal coverings (Clifford-Lipschitz groups) of the orthogonal groups is given in terms of $C P T$-structures which include well-known Shirokov-Dąbrowski PTstructures as a particular case. Quotient Clifford-Lipschitz groups and quotient representations are introduced. It is shown that a complete classification of the quotient groups depends on the structure of various subgroups of the extended automorphism group.
\end{abstract}

\section{Introduction}

From historical point of view Clifford algebras have essentially geometric origin [24, because they are the synthesis of Hamilton quaternion calculus 45] 
and Grassmann Ausdehnungslehre [43, and by this reason they called by Clifford as geometric algebras [25]. Further, Lipschitz [54] showed that Clifford algebras closely related with the study of the rotation groups of multidimensional spaces. After fundamental works of Cartan [16], Witt [83] and Chevalley 20 the Clifford algebra theory takes its modern form [27, 62, 55]. The Pin and Spin groups (Clifford-Lipschitz groups) widely used in algebraic topology 12, 44, 4, 49, 50, 51], in the definition of pinor and spinor structures on the riemannian manifolds [59, 41, 47, 82, 28, 29, 56, 31, 27, 1, 17, 18, 2, spinor bundles 67, 68, 37, 36, 38, and also have great importance in the theory of the Dirac operator on manifolds [8, 7, 77, 35, 3]. The Clifford-Lipschitz groups also intensively used in theoretical physics [23, 32, 34, 69, 33, 10, 19.

Universal coverings of the orthogonal groups allow us to describe discrete symmetries on an equal footing, from the one group theoretical viewpoint. Importance of discrete transformations is well-known, many textbooks on quantum theory began with description of the discrete symmetries, and famous Lüders-Pauli CPT-Theorem is a keystone of quantum field theory.

However, usual practice of definition of the discrete symmetries from the analysis of relativistic wave equations does not give a full and consistent theory of the discrete transformations. In the standard approach, except a well studied case of the spin $j=1 / 2$ (Dirac equation), a situation with the discrete symmetries remains unclear for the fields of higher spin $j>1 / 2$. It is obvious that the main reason of this is an absence of a fully adequate formalism for description of higher-spin fields (all widely accepted higher-spin formalisms such as Rarita-Schwinger approach [64, Bargmann-Wigner [6] and Gel'fand-Yaglom [39] multispinor theories, and also Joos-Weinberg $2(2 j+1)-$ component formalism [48, 81] have many intrinsic contradictions and difficulties). Moreover, Lee and Wick [53] claimed that "the situation is clearly an unsatisfactory one from a fundamental point of view". The first attempt of going out from this situation was initiated by Gel'fand, Minlos and Shapiro in 1958 [40]. In the Gel'fand-Minlos-Shapiro approach the discrete symmetries are represented by outer involutory automorphisms of the Lorentz group (there are also other realizations of the discrete symmetries via the outer automorphisms, see [58, 52, 76]). At present, the Gel'fand-Minlos-Shapiro ideas have been found further development in the works of Buchbinder, Gitman and Shelepin [14, 42], where the discrete symmetries are represented by both outer and inner automorphisms of the Poincaré group.

In 1909, Minkowski showed 60 that a causal structure of the world is described by a 4-dimensional pseudo-Euclidean geometry. In accordance with 60] the quadratic form $x^{2}+y^{2}+z^{2}-c^{2} t^{2}$ remains invariant under the action of linear transformations of the four variables $x, y, z$ and $t$, which form a gen- 
eral Lorentz group $G$. As known, the general Lorentz group $G$ consists of a proper orthochronous Lorentz group $G_{0}$ and three reflections (discrete transformations) $P, T, P T$, where $P$ and $T$ are space and time reversal, and $P T$ is a so-called full reflection. The discrete transformations $P, T$ and $P T$ added to an identical transformation form a finite group. Thus, the general Lorentz group may be represented by a semidirect product $G_{0} \odot\{1, P, T, P T\}$.

In 1958, Shirokov pointed out [74, 75] that an universal covering of the inhomogeneous Lorentz group has eight inequivalent realizations. Later on, in the eighties this idea was applied to a general orthogonal group $O(p, q)$ by Dąbrowski 30. As known, the orthogonal group $O(p, q)$ of the real space $\mathbb{R}^{p, q}$ is represented by the semidirect product of a connected component $O_{0}(p, q)$ and a discrete subgroup $\{1, P, T, P T\}$. Further, a double covering of the orthogonal group $O(p, q)$ is a Clifford-Lipschitz group $\operatorname{Pin}(p, q)$ which is completely constructed within a Clifford algebra $C_{p, q}$. In accordance with squares of elements of the discrete subgroup $\left(a=P^{2}, b=T^{2}, c=(P T)^{2}\right)$ there exist eight double coverings (Da̧browski groups [30]) of the orthogonal group defining by the signatures $(a, b, c)$, where $a, b, c \in\{-,+\}$. Such in brief is a standard description scheme of the discrete transformations. However, in this scheme there is one essential flaw. Namely, the Clifford-Lipschitz group is an intrinsic notion of the algebra $C \ell_{p, q}$ (a set of the all invertible elements of $C \ell_{p, q}$ ), whereas the discrete subgroup is introduced into the standard scheme in an external way, and the choice of the signature $(a, b, c)$ of the discrete subgroup is not determined by the signature of the space $\mathbb{R}^{p, q}$. Moreover, it is suggest by default that for any signature $(p, q)$ of the vector space there exist the all eight kinds of the discrete subgroups. It is obvious that a consistent description of the universal coverings of $O(p, q)$ in terms of the Clifford-Lipschitz groups $\operatorname{Pin}(p, q) \subset C \ell_{p, q}$ can be obtained only in the case when the discrete subgroup $\{1, P, T, P T\}$ is also defined within the algebra $C \ell_{p, q}$. Such a description has been given in the works [78, 79, 80, where the discrete symmetries are represented by fundamental automorphisms of the Clifford algebras. So, the space inversion $P$, time reversal $T$ and their combination $P T$ correspond to an automorphism $\star$ (involution), an antiautomorphism $\sim$ (reversion) and an antiautomorphism $\widetilde{\star}$ (conjugation), respectively. The fundamental automorphisms of the Clifford algebras are compared to elements of the finite group formed by the discrete transformations. In turn, a set of the fundamental automorphisms, added by an identical automorphism, forms a finite group $\operatorname{Aut}(\mathrm{Cl})$, for which in virtue of the Wedderburn-Artin Theorem there exists a matrix representation. In such a way, an isomorphism $\{1, P, T, P T\} \simeq \operatorname{Aut}(C \ell)$ plays a central role and allows us to use methods of the Clifford algebra theory at the study of a group theoretical structure of the discrete transformations. First of all, it allows one to 
classify the discrete groups into Abelian $\mathbb{Z}_{2} \otimes \mathbb{Z}_{2}, \mathbb{Z}_{4}$ and non-Abelian $D_{4}, Q_{4}$ finite groups, and also to establish a dependence between the finite groups and signature of the spaces in case of real numbers. It is shown that the division ring structure of $C_{p, q}$ imposes hard restrictions on existence and choice of the discrete subgroup, and the signature $(a, b, c)$ depends upon the signature of the underlying space $\mathbb{R}^{p, q}$. Moreover, this description allows us to incorporate the Gel'fand-Minlos-Shapiro automorphism theory into Shirokov-Dąbrowski scheme and further to unite them on the basis of the Clifford algebra theory.

Other important discrete symmetry is the charge conjugation $C$. In contrast with the transformations $P, T, P T$, the operation $C$ is not space-time discrete symmetry. This transformation is firstly appearred on the representation spaces of the Lorentz group and its nature is strongly different from other discrete symmetries. For that reason in this work the charge conjugation $C$ is represented by a pseudoautomorphism $\mathcal{A} \rightarrow \overline{\mathcal{A}}$ which is not fundamental automorphism of the Clifford algebra. All spinor representations of the pseudoautomorphism $\mathcal{A} \rightarrow \overline{\mathcal{A}}$ are given in Theorem 3 . An introduction of the transformation $\mathcal{A} \rightarrow \overline{\mathcal{A}}$ allows us to extend the automorphism group $\operatorname{Aut}(C \ell)$ of the Clifford algebra. It is shown that automorphisms $\mathcal{A} \rightarrow \mathcal{A}^{\star}, \mathcal{A} \rightarrow \widetilde{\mathcal{A}}$, $\mathcal{A} \rightarrow \widetilde{\mathcal{A}^{\star}}, \mathcal{A} \rightarrow \overline{\mathcal{A}}, \mathcal{A} \rightarrow \overline{\mathcal{A}^{\star}}, \mathcal{A} \rightarrow \overline{\widetilde{\mathcal{A}}}$ and $\mathcal{A} \rightarrow \overline{\widetilde{\mathcal{A}^{\star}}}$ form a finite group of order

8 (an extended automorphism group $\left.\operatorname{Ext}(C \ell)=\left\{\mathrm{Id}, \star,^{\sim}, \widetilde{\star},-, \bar{\star}, \bar{\sim}, \overline{\widetilde{\star}}\right\}\right)$. The group $\operatorname{Ext}(C \ell)$ is isomorphic to a $C P T$-group $\{1, P, T, P T, C, C P, C T, C P T\}$. There exist isomorphisms between $\operatorname{Ext}(C \ell)$ and finite groups of order 8. A full number of different realizations of $\operatorname{Ext}(C \ell)$ is equal to 64 . This result allows us to define extended universal coverings (CPT-structures) of the orthogonal groups. It is shown that the eight Shirokov-Dąbrowski $P T$-structures present a particular case of general $C P T$-structures.

Quotient representations of the group $\operatorname{Pin}(n, \mathbb{C})$ and $\operatorname{Pin}(p, q)$ correspond to the types $n \equiv 1(\bmod 2)(\mathbb{F}=\mathbb{C})$ and $p-q \equiv 1,5(\bmod 8)(\mathbb{F}=\mathbb{R})$, respectively. An explicit form of the quotient representations is given in Theorem 14. It is proved that a classification of the quotient groups depends on the structure of different subgroups of $\operatorname{Ext}(C \ell)$.

\section{Clifford algebras and discrete symmetries}

In this section we will consider some basic facts concerning Clifford algebras which we will widely use below. Let $\mathbb{F}$ be a field of characteristic $0(\mathbb{F}=\mathbb{R}, \mathbb{F}=$ $\mathbb{C})$, where $\mathbb{R}$ and $\mathbb{C}$ are the fields of real and complex numbers, respectively. A Clifford algebra $C l$ over a field $\mathbb{F}$ is an algebra with $2^{n}$ basis elements: $\mathbf{e}_{0}$ (unit of the algebra) $\mathbf{e}_{1}, \mathbf{e}_{2}, \ldots, \mathbf{e}_{n}$ and products of the one-index elements 
$\mathbf{e}_{i_{1} i_{2} \ldots i_{k}}=\mathbf{e}_{i_{1}} \mathbf{e}_{i_{2}} \ldots \mathbf{e}_{i_{k}}$. Over the field $\mathbb{F}=\mathbb{R}$ the Clifford algebra is denoted as $C \ell_{p, q}$, where the indices $p$ and $q$ correspond to the indices of the quadratic form

$$
Q=x_{1}^{2}+\ldots+x_{p}^{2}-\ldots-x_{p+q}^{2}
$$

of a vector space $V$ associated with $C \ell_{p, q}$. The multiplication law of $C \ell_{p, q}$ is defined by a following rule:

$$
\mathbf{e}_{i}^{2}=\sigma(p-i) \mathbf{e}_{0}, \quad \mathbf{e}_{i} \mathbf{e}_{j}=-\mathbf{e}_{j} \mathbf{e}_{i}
$$

where

$$
\sigma(n)= \begin{cases}-1 & \text { if } n \leq 0 \\ +1 & \text { if } n>0\end{cases}
$$

The square of a volume element $\omega=\mathbf{e}_{12 \ldots n}(n=p+q)$ plays an important role in the theory of Clifford algebras,

$$
\omega^{2}=\left\{\begin{array}{lll}
-1 & \text { if } p-q \equiv 2,3,6,7 \quad(\bmod 8) \\
+1 & \text { if } p-q \equiv 0,1,4,5 \quad(\bmod 8)
\end{array}\right.
$$

A center $\mathbf{Z}_{p, q}$ of the algebra $C \ell_{p, q}$ consists of the unit $\mathbf{e}_{0}$ and the volume element $\omega$. The element $\omega=\mathbf{e}_{12 \ldots n}$ belongs to a center when $n$ is odd. Indeed,

$$
\begin{aligned}
& \mathbf{e}_{12 \ldots n} \mathbf{e}_{i}=(-1)^{n-i} \sigma(q-i) \mathbf{e}_{12 \ldots i-1 i+1 \ldots n}, \\
& \mathbf{e}_{i} \mathbf{e}_{12 \ldots n}=(-1)^{i-1} \sigma(q-i) \mathbf{e}_{12 \ldots i-1 i+1 \ldots n},
\end{aligned}
$$

therefore, $\omega \in \mathbf{Z}_{p, q}$ if and only if $n-i \equiv i-1(\bmod 2)$, that is, $n$ is odd. Further, using (31) we obtain

$$
\mathbf{Z}_{p, q}=\left\{\begin{aligned}
1 & \text { if } p-q \equiv 0,2,4,6 \quad(\bmod 8) \\
1, \omega & \text { if } p-q \equiv 1,3,5,7 \quad(\bmod 8)
\end{aligned}\right.
$$

An arbitrary element $\mathcal{A}$ of the algebra $C \ell_{p, q}$ is represented by a following formal polynomial

$$
\begin{gathered}
\mathcal{A}=a^{0} \mathbf{e}_{0}+\sum_{i=1}^{n} a^{i} \mathbf{e}_{i}+\sum_{i=1}^{n} \sum_{j=1}^{n} a^{i j} \mathbf{e}_{i j}+\ldots+\sum_{i_{1}=1}^{n} \ldots \sum_{i_{k}=1}^{n} a^{i_{1} \ldots i_{k}} \mathbf{e}_{i_{1} \ldots i_{k}}+ \\
+\ldots+a^{12 \ldots n} \mathbf{e}_{12 \ldots n}=\sum_{k=0}^{n} a^{i_{1} i_{2} \ldots i_{k}} \mathbf{e}_{i_{1} i_{2} \ldots i_{k}}
\end{gathered}
$$

In Clifford algebra $C \ell$ there exist four fundamental automorphisms.

1) Identity: An automorphism $\mathcal{A} \rightarrow \mathcal{A}$ and $\mathbf{e}_{i} \rightarrow \mathbf{e}_{i}$. 
This automorphism is an identical automorphism of the algebra $C \ell . \mathcal{A}$ is an arbitrary element of $C \ell$.

2) Involution: An automorphism $\mathcal{A} \rightarrow \mathcal{A}^{\star}$ and $\mathbf{e}_{i} \rightarrow-\mathbf{e}_{i}$.

In more details, for an arbitrary element $\mathcal{A} \in C$ there exists a decomposition $\mathcal{A}=\mathcal{A}^{\prime}+\mathcal{A}^{\prime \prime}$, where $\mathcal{A}^{\prime}$ is an element consisting of homogeneous odd elements, and $\mathcal{A}^{\prime \prime}$ is an element consisting of homogeneous even elements, respectively. Then the automorphism $\mathcal{A} \rightarrow \mathcal{A}^{\star}$ is such that the element $\mathcal{A}^{\prime \prime}$ is not changed, and the element $\mathcal{A}^{\prime}$ changes sign: $\mathcal{A}^{\star}=-\mathcal{A}^{\prime}+\mathcal{A}^{\prime \prime}$. If $\mathcal{A}$ is a homogeneous element, then

$$
\mathcal{A}^{\star}=(-1)^{k} \mathcal{A}
$$

where $k$ is a degree of the element. It is easy to see that the automorphism $\mathcal{A} \rightarrow \mathcal{A}^{\star}$ may be expressed via the volume element $\omega=\mathbf{e}_{12 \ldots p+q}$ :

$$
\mathcal{A}^{\star}=\omega \mathcal{A} \omega^{-1},
$$

where $\omega^{-1}=(-1)^{\frac{(p+q)(p+q-1)}{2}} \omega$. When $k$ is odd, the basis elements $\mathbf{e}_{i_{1} i_{2} \ldots i_{k}}$ the sign changes, and when $k$ is even, the sign is not changed.

3) Reversion: An antiautomorphism $\mathcal{A} \rightarrow \widetilde{\mathcal{A}}$ and $\mathbf{e}_{i} \rightarrow \mathbf{e}_{i}$.

The antiautomorphism $\mathcal{A} \rightarrow \widetilde{\mathcal{A}}$ is a reversion of the element $\mathcal{A}$, that is the substitution of each basis element $\mathbf{e}_{i_{1} i_{2} \ldots i_{k}} \in \mathcal{A}$ by the element $\mathbf{e}_{i_{k} i_{k-1} \ldots i_{1}}$ :

$$
\mathbf{e}_{i_{k} i_{k-1} \ldots i_{1}}=(-1)^{\frac{k(k-1)}{2}} \mathbf{e}_{i_{1} i_{2} \ldots i_{k}} .
$$

Therefore, for any $\mathcal{A} \in C_{p, q}$ we have

$$
\widetilde{\mathcal{A}}=(-1)^{\frac{k(k-1)}{2}} \mathcal{A}
$$

4) Conjugation: An antiautomorphism $\mathcal{A} \rightarrow \widetilde{\mathcal{A}^{\star}}$ and $\mathbf{e}_{i} \rightarrow-\mathbf{e}_{i}$.

This antiautomorphism is a composition of the antiautomorphism $\mathcal{A} \rightarrow \widetilde{\mathcal{A}}$ with the automorphism $\mathcal{A} \rightarrow \mathcal{A}^{\star}$. In the case of a homogeneous element from the formulae (15) and (77), it follows

$$
\widetilde{\mathcal{A}^{\star}}=(-1)^{\frac{k(k+1)}{2}} \mathcal{A} .
$$

As known, the complex algebra $\mathbb{C}_{n}$ is associated with a complex vector space $\mathbb{C}^{n}$. Let $n=p+q$, then an extraction operation of the real subspace $\mathbb{R}^{p, q}$ in $\mathbb{C}^{n}$ forms the foundation of definition of the discrete transformation known in physics as a charge conjugation $C$. Indeed, let $\left\{\mathbf{e}_{1}, \ldots, \mathbf{e}_{n}\right\}$ be an orthobasis in 
the space $\mathbb{C}^{n}, \mathbf{e}_{i}^{2}=1$. Let us remain the first $p$ vectors of this basis unchanged, and other $q$ vectors multiply by the factor $i$. Then the basis

$$
\left\{\mathbf{e}_{1}, \ldots, \mathbf{e}_{p}, i \mathbf{e}_{p+1}, \ldots, i \mathbf{e}_{p+q}\right\}
$$

allows one to extract the subspace $\mathbb{R}^{p, q}$ in $\mathbb{C}^{n}$. Namely, for the vectors $\mathbb{R}^{p, q}$ we take the vectors of $\mathbb{C}^{n}$ which decompose on the basis (9) with real coefficients. In such a way we obtain a real vector space $\mathbb{R}^{p, q}$ endowed (in general case) with a non-degenerate quadratic form

$$
Q(x)=x_{1}^{2}+x_{2}^{2}+\ldots+x_{p}^{2}-x_{p+1}^{2}-x_{p+2}^{2}-\ldots-x_{p+q}^{2},
$$

where $x_{1}, \ldots, x_{p+q}$ are coordinates of the vector $\mathbf{x}$ in the basis (19). It is easy to see that the extraction of $\mathbb{R}^{p, q}$ in $\mathbb{C}^{n}$ induces an extraction of a real subalgebra $\ell_{p, q}$ in $\mathbb{C}_{n}$. Therefore, any element $\mathcal{A} \in \mathbb{C}_{n}$ can be unambiguously represented in the form

$$
\mathcal{A}=\mathcal{A}_{1}+i \mathcal{A}_{2}
$$

where $\mathcal{A}_{1}, \mathcal{A}_{2} \in C_{p, q}$. The one-to-one mapping

$$
\mathcal{A} \longrightarrow \overline{\mathcal{A}}=\mathcal{A}_{1}-i \mathcal{A}_{2}
$$

transforms the algebra $\mathbb{C}_{n}$ into itself with preservation of addition and multiplication operations for the elements $\mathcal{A}$; the operation of multiplication of the element $\mathcal{A}$ by the number transforms to an operation of multiplication by the complex conjugate number. Any mapping of $\mathbb{C}_{n}$ satisfying these conditions is called a pseudoautomorphism. Thus, the extraction of the subspace $\mathbb{R}^{p, q}$ in the space $\mathbb{C}^{n}$ induces in the algebra $\mathbb{C}_{n}$ a pseudoautomorphism $\mathcal{A} \rightarrow \overline{\mathcal{A}}$ [65, 66].

One of the most fundamental theorems in the theory of associative algebras is

Theorem 1 (Wedderburn-Artin). Any finite-dimensional associative simple algebra $\mathfrak{A}$ over the field $\mathbb{F}$ is isomorphic to a full matrix algebra $\mathrm{M}_{n}(\mathbb{K})$, where a natural number $n$ defined unambiguously, and a division ring $\mathbb{K}$ defined with an accuracy of isomorphism.

In accordance with this theorem all properties of the initial algebra $\mathfrak{A}$ are isomorphically transferred to the matrix algebra $\mathrm{M}_{n}(\mathbb{K})$. Later on we will widely use this theorem. In turn, for the Clifford algebra $C \ell_{p, q}$ over the field $\mathbb{F}=\mathbb{R}$ we have an isomorphism $C \ell_{p, q} \simeq \operatorname{End}_{\mathbb{K}}\left(I_{p, q}\right) \simeq \mathrm{M}_{2^{m}}(\mathbb{K})$, where $m=\frac{p+q}{2}$, $I_{p, q}=C \ell_{p, q} f$ is a minimal left ideal of $C \ell_{p, q}$, and $\mathbb{K}=f C \ell_{p, q} f$ is a division ring of $C \ell_{p, q}$. The primitive idempotent of the algebra $C \ell_{p, q}$ has a form

$$
f=\frac{1}{2}\left(1 \pm \mathbf{e}_{\alpha_{1}}\right) \frac{1}{2}\left(1 \pm \mathbf{e}_{\alpha_{2}}\right) \cdots \frac{1}{2}\left(1 \pm \mathbf{e}_{\alpha_{k}}\right),
$$


where $\mathbf{e}_{\alpha_{1}}, \mathbf{e}_{\alpha_{2}}, \ldots, \mathbf{e}_{\alpha_{k}}$ are commuting elements with square 1 of the canonical basis of $C \ell_{p, q}$ generating a group of order $2^{k}$. The values of $k$ are defined by a formula $k=q-r_{q-p}$, where $r_{i}$ are the Radon-Hurwitz numbers [63, 46], values of which form a cycle of the period 8: $r_{i+8}=r_{i}+4$. The values of all $r_{i}$ are

\begin{tabular}{lllllllll}
$i$ & 0 & 1 & 2 & 3 & 4 & 5 & 6 & 7 \\
\hline$r_{i}$ & 0 & 1 & 2 & 2 & 3 & 3 & 3 & 3
\end{tabular}.

The all Clifford algebras $C \ell_{p, q}$ over the field $\mathbb{F}=\mathbb{R}$ are divided into eight different types with a following division ring structure:

I. Central simple algebras.

1) Two types $p-q \equiv 0,2(\bmod 8)$ with a division $\operatorname{ring} \mathbb{K} \simeq \mathbb{R}$.

2) Two types $p-q \equiv 3,7(\bmod 8)$ with a division $\operatorname{ring} \mathbb{K} \simeq \mathbb{C}$.

3) Two types $p-q \equiv 4,6(\bmod 8)$ with a division $\operatorname{ring} \mathbb{K} \simeq \mathbb{H}$.

II. Semi-simple algebras.

4) The type $p-q \equiv 1(\bmod 8)$ with a double division $\operatorname{ring} \mathbb{K} \simeq \mathbb{R} \oplus \mathbb{R}$.

5) The type $p-q \equiv 5(\bmod 8)$ with a double quaternionic division ring $\mathbb{K} \simeq \mathbb{H} \oplus \mathbb{H}$.

The Table 1 (Budinich-Trautman Periodic Table [15]) explicitly shows a distribution of the real Clifford algebras in dependence on the division ring structure, here ${ }^{2} \mathbb{R}(n)=\mathbb{R}(n) \oplus \mathbb{R}(n)$ and ${ }^{2} \mathbb{H}(n)=\mathbb{H}(n) \oplus \mathbb{H}(n)$.

Over the field $\mathbb{F}=\mathbb{C}$ there is an isomorphism $\mathbb{C}_{n} \simeq \mathrm{M}_{2^{n / 2}}(\mathbb{C})$ and there are two different types of complex Clifford algebras $\mathbb{C}_{n}: n \equiv 0(\bmod 2)$ and $n \equiv 1$ $(\bmod 2)$.

When $C \ell_{p, q}$ is simple, then the map

$$
C \ell_{p, q} \stackrel{\gamma}{\longrightarrow} \operatorname{End}_{\mathbb{K}}(\mathbb{S}), \quad u \longrightarrow \gamma(u), \quad \gamma(u) \psi=u \psi
$$

gives an irreducible and faithful representation of $C \ell_{p, q}$ in the spinspace $\mathbb{S}_{2^{m}}(\mathbb{K}) \simeq I_{p, q}=C \ell_{p, q} f$, where $\psi \in \mathbb{S}_{2^{m}}, m=\frac{p+q}{2}$.

On the other hand, when $C \ell_{p, q}$ is semi-simple, then the map

$$
C \ell_{p, q} \stackrel{\gamma}{\longrightarrow} \operatorname{End}_{\mathbb{K} \oplus \hat{\mathbb{K}}}(\mathbb{S} \oplus \hat{\mathbb{S}}), \quad u \longrightarrow \gamma(u), \quad \gamma(u) \psi=u \psi
$$

gives a faithful but reducible representation of $C_{p, q}$ in the double spinspace $\mathbb{S} \oplus \hat{\mathbb{S}}$, where $\hat{\mathbb{S}}=\{\hat{\psi} \mid \psi \in \mathbb{S}\}$. In this case, the ideal $\mathbb{S} \oplus \hat{\mathbb{S}}$ possesses a right 
Table 1. Distribution of the real Clifford algebras.

\begin{tabular}{|c|c|c|c|c|c|c|c|c|c|}
\hline $\mathrm{p}$ & 0 & 1 & 2 & 3 & 4 & 5 & 6 & 7 & $\ldots$ \\
\hline$q$ & & & & & & & & & \\
\hline 0 & $\mathbb{R}$ & ${ }^{2} \mathbb{R}$ & $\mathbb{R}(2)$ & $\mathbb{C}(2)$ & $\mathbb{H}(2)$ & ${ }^{2} \mathbb{H}(2)$ & $\mathbb{H}(4)$ & $\mathbb{C}(8)$ & $\ldots$ \\
\hline 1 & $\mathbb{C}$ & $\mathbb{R}(2)$ & ${ }^{2} \mathbb{R}(2)$ & $\mathbb{R}(4)$ & $\mathbb{C}(4)$ & $\mathbb{H}(4)$ & ${ }^{2} \mathbb{H}(4)$ & $\mathbb{H}(8)$ & $\because$ \\
\hline 2 & $\mathbb{H}$ & $\mathbb{C}(2)$ & $\mathbb{R}(4)$ & ${ }^{2} \mathbb{R}(4)$ & $\mathbb{R}(8)$ & $\mathbb{C}(8)$ & $\mathbb{H}(8)$ & ${ }^{2} \mathbb{H}(8)$ & . \\
\hline 3 & ${ }^{2} \mathbb{H}$ & $\mathbb{H}(2)$ & $\mathbb{C}(4)$ & $\mathbb{R}(8)$ & ${ }^{2} \mathbb{R}(8)$ & $\mathbb{R}(16)$ & $\mathbb{C}(16)$ & $\mathbb{H}(16)$ & \\
\hline 4 & $\mathbb{H}(2)$ & ${ }^{2} \mathbb{H}(2)$ & $\mathbb{H}(4)$ & $\mathbb{C}(8)$ & $\mathbb{R}(16)$ & ${ }^{2} \mathbb{R}(16)$ & $\mathbb{R}(32)$ & $\mathbb{C}(32)$ & . \\
\hline 5 & $\mathbb{C}(4)$ & $\mathbb{H}(4)$ & ${ }^{2} \mathbb{H}(4)$ & $\mathbb{H}(8)$ & $\mathbb{C}(16)$ & $\mathbb{R}(32)$ & ${ }^{2} \mathbb{R}(32)$ & $\mathbb{R}(64)$ & . \\
\hline 6 & $\mathbb{R}(8)$ & $\mathbb{C}(8)$ & $\mathbb{H}(8)$ & ${ }^{2} \mathbb{H}(8)$ & $\mathbb{H}(16)$ & $\mathbb{C}(32)$ & $\mathbb{R}(64)$ & ${ }^{2} \mathbb{R}(64)$ & . \\
\hline 7 & ${ }^{2} \mathbb{R}(8)$ & $\mathbb{R}(16)$ & $\mathbb{C}(16)$ & $\mathbb{H}(16)$ & ${ }^{2} \mathbb{H}(16)$ & $\mathbb{H}(32)$ & $\mathbb{C}(64)$ & $\mathbb{R}(128)$ & . \\
\hline$\vdots$ & $\vdots$ & $\vdots$ & $\vdots$ & $\vdots$ & $\vdots$ & $\vdots$ & $\vdots$ & & \\
\hline
\end{tabular}

$\mathbb{K} \oplus \hat{\mathbb{K}}$-linear structure, $\hat{\mathbb{K}}=\{\hat{\lambda} \mid \lambda \in \mathbb{K}\}$, and $\mathbb{K} \oplus \hat{\mathbb{K}}$ is isomorphic to the double division $\operatorname{ring} \mathbb{R} \oplus \mathbb{R}$ when $p-q \equiv 1(\bmod 8)$ or to $\mathbb{H} \oplus \mathbb{H}$ when $p-q \equiv 5$ $(\bmod 8)$. The map $\gamma$ in (11) and (12) defines the so called left-regular spinor representation of $C \ell(Q)$ in $\mathbb{S}$ and $\mathbb{S} \oplus \hat{\mathbb{S}}$, respectively. Furthermore, $\gamma$ is faithful which means that $\gamma$ is an algebra monomorphism. In (111), $\gamma$ is irreducible which means that $\mathbb{S}$ possesses no proper (that is, $\neq 0, \mathbb{S}$ ) invariant subspaces under the left action of $\gamma(u), u \in C_{p, q}$. Representation $\gamma$ in (12) is therefore reducible since $\{(\psi, 0) \mid \psi \in \mathbb{S}\}$ and $\{(0, \hat{\psi}) \mid \hat{\psi} \in \hat{\mathbb{S}}\}$ are two proper subspaces of $\mathbb{S} \oplus \hat{\mathbb{S}}$ invariant under $\gamma(u)$ (see [55, 27, 62]).

\subsection{Salingaros groups}

The structure of the Clifford algebras admits a very elegant description in terms of finite groups [70, 71, 72]. In accordance with the multiplication law (11) the basis elements of $C \ell_{p, q}$ form a finite group of order $2^{n+1}$,

$$
G(p, q)=\left\{ \pm 1, \pm \mathbf{e}_{i}, \pm \mathbf{e}_{i} \mathbf{e}_{j}, \pm \mathbf{e}_{i} \mathbf{e}_{j} \mathbf{e}_{k}, \ldots, \pm \mathbf{e}_{1} \mathbf{e}_{2} \cdots \mathbf{e}_{n}\right\} \quad(i<j<k<\ldots) .
$$

Salingaros showed [70, 71] that there exist five distinct types of finite groups (13) that arise from Clifford algebras. In [70, 71] they were called 'vee groups' and were labelled as

$$
N_{\text {odd }}, N_{\text {even }}, \Omega_{\text {odd }}, \Omega_{\text {even }}, S_{k}
$$


The odd $N$-groups correspond to real spinors, for example, $N_{1}$ is related to real 2-spinors, and $N_{3}$ is the group of the real Majorana matrices. The even $N$ groups define the quaternionic groups. The $S$-groups are the 'spinor groups' $\left(S_{k}=N_{2 k} \otimes \mathbb{C} \simeq N_{2 k-1} \otimes \mathbb{C}\right): S_{1}$ is the group of the complex Pauli matrices, and $S_{2}$ is the group of the Dirac matrices. Furthermore, the $\Omega$-groups are double copies of the $N$-groups and can be written as a direct product of the $N$-groups with the group of two elements $\mathbb{Z}_{2}$ :

$$
\Omega_{k}=N_{k} \otimes \mathbb{Z}_{2}
$$

Let us consider now several simplest examples of the groups (14). First of all, a finite group corresponding to the Clifford algebra $C \ell_{0,0}$ with an arbitrary element $\mathcal{A}=a^{0}$ and division $\operatorname{ring} \mathbb{K} \simeq \mathbb{R}(p-q \equiv 0(\bmod 8))$ is a cyclic group $\mathbb{Z}_{2}=\{1,-1\}$ with the following multiplication table

\begin{tabular}{|c||c|c|}
\hline & 1 & -1 \\
\hline \hline 1 & 1 & -1 \\
\hline-1 & -1 & 1 \\
\hline
\end{tabular}

It is easy to see that in accordance with (14) the finite group corresponding to $C \ell_{0,0}$ is $N_{0}$-group $\left(N_{0}=\mathbb{Z}_{2}\right)$.

A further Clifford algebra is $C \ell_{1,0}: \mathcal{A}=a^{0}+a^{1} \mathbf{e}_{1}, \mathbf{e}_{1}^{2}=1, \mathbb{K} \simeq \mathbb{R} \oplus \mathbb{R}$, $p-q \equiv 1(\bmod 8)$. In this case the basis elements of $C \ell_{1,0}$ form the Gauss-Klein four-group $\mathbb{Z}_{2} \otimes \mathbb{Z}_{2}=\left\{1,-1, \mathbf{e}_{1},-\mathbf{e}_{1}\right\}$. The multiplication table of $\mathbb{Z}_{2} \otimes \mathbb{Z}_{2}$ has a form

\begin{tabular}{|c||c|c|c|c|}
\hline & 1 & -1 & $\mathbf{e}_{1}$ & $-\mathbf{e}_{1}$ \\
\hline \hline 1 & 1 & -1 & $\mathbf{e}_{1}$ & $-\mathbf{e}_{1}$ \\
\hline-1 & -1 & 1 & $-\mathbf{e}_{1}$ & $\mathbf{e}_{1}$ \\
\hline $\mathbf{e}_{1}$ & $\mathbf{e}_{1}$ & $-\mathbf{e}_{1}$ & 1 & -1 \\
\hline$-\mathbf{e}_{1}$ & $-\mathbf{e}_{1}$ & $\mathbf{e}_{1}$ & -1 & 1 \\
\hline
\end{tabular}

In accordance with (15) we have here a first $\Omega$-group: $\Omega_{0}=N_{0} \otimes \mathbb{Z}_{2}=$ $N_{0} \otimes N_{0}=\mathbb{Z}_{2} \otimes \mathbb{Z}_{2}$.

The algebra $C \ell_{0,1}$ with $\mathcal{A}=a^{0}+a^{1} \mathbf{e}_{1}, \mathbf{e}_{1}^{2}=-1(\mathbb{K} \simeq \mathbb{C}, p-q \equiv 7$ $(\bmod 8))$ corresponds to the complex group $\mathbb{Z}_{4}=\left\{1,-1, \mathbf{e}_{1},-\mathbf{e}_{1}\right\}$ with the 
multiplication table

\begin{tabular}{|c||c|c|c|c|}
\hline & 1 & -1 & $\mathbf{e}_{1}$ & $-\mathbf{e}_{1}$ \\
\hline \hline 1 & 1 & -1 & $\mathbf{e}_{1}$ & $-\mathbf{e}_{1}$ \\
\hline-1 & -1 & 1 & $-\mathbf{e}_{1}$ & $\mathbf{e}_{1}$ \\
\hline $\mathbf{e}_{1}$ & $\mathbf{e}_{1}$ & $-\mathbf{e}_{1}$ & -1 & 1 \\
\hline$-\mathbf{e}_{1}$ & $-\mathbf{e}_{1}$ & $\mathbf{e}_{1}$ & 1 & -1 \\
\hline
\end{tabular}

It is easy to see that in accordance with Salingaros classification this group is a first $S$-group: $S_{0}=\mathbb{Z}_{4}$.

The three finite groups considered previously are Abelian groups. All other Salingaros groups (14) are non-Abelian. The first non-Abelian Salingaros group correspond to the algebra $C \ell_{2,0}$ with an arbitrary element $\mathcal{A}=a^{0}+a^{1} \mathbf{e}_{1}+$ $a^{2} \mathbf{e}_{2}+a^{12} \mathbf{e}_{12}$ and $\mathbf{e}_{1}^{2}=\mathbf{e}_{2}^{2}=1, \mathbf{e}_{12}^{2}=-1(p-q \equiv 2(\bmod 8), \mathbb{K} \simeq \mathbb{R})$. The basis elements of $C \ell_{2,0}$ form a dihedral group $D_{4}=\left\{1,-1, \mathbf{e}_{1},-\mathbf{e}_{1}, \mathbf{e}_{2},-\mathbf{e}_{2}, \mathbf{e}_{12},-\mathbf{e}_{12}\right\}$ with the table

\begin{tabular}{|c||c|c|c|c|c|c|c|c|}
\hline & 1 & -1 & $\mathbf{e}_{1}$ & $-\mathbf{e}_{1}$ & $\mathbf{e}_{2}$ & $-\mathbf{e}_{2}$ & $\mathbf{e}_{12}$ & $-\mathbf{e}_{12}$ \\
\hline \hline 1 & 1 & -1 & $\mathbf{e}_{1}$ & $-\mathbf{e}_{1}$ & $\mathbf{e}_{2}$ & $-\mathbf{e}_{2}$ & $\mathbf{e}_{12}$ & $-\mathbf{e}_{12}$ \\
\hline-1 & -1 & 1 & $-\mathbf{e}_{1}$ & $\mathbf{e}_{1}$ & $-\mathbf{e}_{2}$ & $\mathbf{e}_{2}$ & $-\mathbf{e}_{12}$ & $\mathbf{e}_{12}$ \\
\hline $\mathbf{e}_{1}$ & $\mathbf{e}_{1}$ & $-\mathbf{e}_{1}$ & 1 & -1 & $\mathbf{e}_{12}$ & $-\mathbf{e}_{12}$ & $\mathbf{e}_{2}$ & $-\mathbf{e}_{2}$ \\
\hline$-\mathbf{e}_{1}$ & $-\mathbf{e}_{1}$ & $\mathbf{e}_{1}$ & -1 & 1 & $-\mathbf{e}_{12}$ & $\mathbf{e}_{12}$ & $-\mathbf{e}_{2}$ & $\mathbf{e}_{2}$ \\
\hline $\mathbf{e}_{2}$ & $\mathbf{e}_{2}$ & $-\mathbf{e}_{2}$ & $-\mathbf{e}_{12}$ & $\mathbf{e}_{12}$ & 1 & -1 & $-\mathbf{e}_{1}$ & $\mathbf{e}_{1}$ \\
\hline$-\mathbf{e}_{2}$ & $-\mathbf{e}_{2}$ & $\mathbf{e}_{2}$ & $\mathbf{e}_{12}$ & $-\mathbf{e}_{12}$ & -1 & 1 & $\mathbf{e}_{1}$ & $-\mathbf{e}_{1}$ \\
\hline $\mathbf{e}_{12}$ & $\mathbf{e}_{12}$ & $-\mathbf{e}_{12}$ & $-\mathbf{e}_{2}$ & $\mathbf{e}_{2}$ & $\mathbf{e}_{1}$ & $-\mathbf{e}_{1}$ & -1 & 1 \\
\hline$-\mathbf{e}_{12}$ & $-\mathbf{e}_{12}$ & $\mathbf{e}_{12}$ & $\mathbf{e}_{2}$ & $-\mathbf{e}_{2}$ & $-\mathbf{e}_{1}$ & $\mathbf{e}_{1}$ & 1 & -1 \\
\hline
\end{tabular}

It is a first $N_{\text {odd }}$-group: $N_{1}=D_{4}$. It is easy to verify that we come to the same group $N_{1}=D_{4}$ for the algebra $C \ell_{1,1}$ with the ring $\mathbb{K} \simeq \mathbb{R}, p-q \equiv 0$ $(\bmod 8)$.

The following non-Abelian finite group we obtain for the algebra $C \ell_{0,2}$ with a quaternionic $\operatorname{ring} \mathbb{K} \simeq \mathbb{H}, p-q \equiv 6(\bmod 8)$. In this case the basis elements of $C \ell_{0,2}$ form a quaternionic group $Q_{4}=\left\{ \pm 1, \pm \mathbf{e}_{1}, \pm \mathbf{e}_{2}, \pm \mathbf{e}_{12}\right\}$ with 
the multiplication table

\begin{tabular}{|c||c|c|c|c|c|c|c|c|}
\hline & 1 & -1 & $\mathbf{e}_{1}$ & $-\mathbf{e}_{1}$ & $\mathbf{e}_{2}$ & $-\mathbf{e}_{2}$ & $\mathbf{e}_{12}$ & $-\mathbf{e}_{12}$ \\
\hline \hline 1 & 1 & -1 & $\mathbf{e}_{1}$ & $-\mathbf{e}_{1}$ & $\mathbf{e}_{2}$ & $-\mathbf{e}_{2}$ & $\mathbf{e}_{12}$ & $-\mathbf{e}_{12}$ \\
\hline-1 & -1 & 1 & $-\mathbf{e}_{1}$ & $\mathbf{e}_{1}$ & $-\mathbf{e}_{2}$ & $\mathbf{e}_{2}$ & $-\mathbf{e}_{12}$ & $\mathbf{e}_{12}$ \\
\hline $\mathbf{e}_{1}$ & $\mathbf{e}_{1}$ & $-\mathbf{e}_{1}$ & -1 & 1 & $\mathbf{e}_{12}$ & $-\mathbf{e}_{12}$ & $-\mathbf{e}_{2}$ & $\mathbf{e}_{2}$ \\
\hline$-\mathbf{e}_{1}$ & $-\mathbf{e}_{1}$ & $\mathbf{e}_{1}$ & 1 & -1 & $-\mathbf{e}_{12}$ & $\mathbf{e}_{12}$ & $\mathbf{e}_{2}$ & $-\mathbf{e}_{2}$ \\
\hline $\mathbf{e}_{2}$ & $\mathbf{e}_{2}$ & $-\mathbf{e}_{2}$ & $-\mathbf{e}_{12}$ & $\mathbf{e}_{12}$ & -1 & 1 & $\mathbf{e}_{1}$ & $-\mathbf{e}_{1}$ \\
\hline$-\mathbf{e}_{2}$ & $-\mathbf{e}_{2}$ & $\mathbf{e}_{2}$ & $\mathbf{e}_{12}$ & $-\mathbf{e}_{12}$ & 1 & -1 & $-\mathbf{e}_{1}$ & $\mathbf{e}_{1}$ \\
\hline $\mathbf{e}_{12}$ & $\mathbf{e}_{12}$ & $-\mathbf{e}_{12}$ & $\mathbf{e}_{2}$ & $-\mathbf{e}_{2}$ & $-\mathbf{e}_{1}$ & $\mathbf{e}_{1}$ & -1 & 1 \\
\hline$-\mathbf{e}_{12}$ & $-\mathbf{e}_{12}$ & $\mathbf{e}_{12}$ & $-\mathbf{e}_{2}$ & $\mathbf{e}_{2}$ & $\mathbf{e}_{1}$ & $-\mathbf{e}_{1}$ & 1 & -1 \\
\hline
\end{tabular}

It is a first $N_{\text {even }}$-group: $N_{2}=Q_{4}$.

Now we can to establish a relationship between the finite groups and division ring structures of $C \ell_{p, q}$. It is easy to see that the five distinct types of Salingaros groups correspond to the five division rings of the real Clifford algebras as follows

$$
\begin{aligned}
N_{\text {odd }} & \leftrightarrow \quad C_{p, q}, p-q \equiv 0,2 \quad(\bmod 8), \mathbb{K} \simeq \mathbb{R} ; \\
N_{\text {even }} & \leftrightarrow \quad C_{p, q}, p-q \equiv 4,6 \quad(\bmod 8), \mathbb{K} \simeq \mathbb{H} ; \\
\Omega_{\text {odd }} & \leftrightarrow \quad C_{p, q}, p-q \equiv 1 \quad(\bmod 8), \mathbb{K} \simeq \mathbb{R} \oplus \mathbb{R} ; \\
\Omega_{\text {even }} & \leftrightarrow \quad C_{p, q}, p-q \equiv 5 \quad(\bmod 8), \mathbb{K} \simeq \mathbb{H} \oplus \mathbb{H} ; \\
S_{k} & \leftrightarrow \quad \ell_{p, q}, p-q \equiv 3,7 \quad(\bmod 8), \mathbb{K} \simeq \mathbb{C} .
\end{aligned}
$$

Therefore, the Periodic Table can be rewritten in terms of finite group structure (see the Table 2). Further, in accordance with (4) a center $\mathbf{Z}_{p, q}$ of the algebra $C \ell_{p, q}$ consists of the unit if $p-q \equiv 0,2,4,6(\bmod 8)$ and the elements $1, \omega=$ $\mathbf{e}_{12 \ldots n}$ if $p-q \equiv 1,3,5,7(\bmod 8)$. Let $\mathbf{Z}(p, q) \subset C \ell_{p, q}$ be a center of the finite group (13). In such a way, we have three distinct realizations of the center $\mathbf{Z}(p, q)$ :

$$
\begin{aligned}
& \mathbf{Z}(p, q)=\{1,-1\} \simeq \mathbb{Z}_{2} \text { if } p-q \equiv 0,2,4,6 \quad(\bmod 8) ; \\
& \mathbf{Z}(p, q)=\{1,-1, \omega,-\omega\} \simeq \mathbb{Z}_{2} \otimes \mathbb{Z}_{2} \text { if } p-q \equiv 1,5 \quad(\bmod 8) ; \\
& \mathbf{Z}(p, q)=\{1,-1, \omega,-\omega\} \simeq \mathbb{Z}_{4} \text { if } p-q \equiv 3,7 \quad(\bmod 8) .
\end{aligned}
$$

The Abelian groups $\mathbf{Z}(p, q)$ are the subgroups of the Salingaros groups (14). Namely, $N$-groups have the center $\mathbb{Z}_{2}, \Omega$-groups have the center $\mathbb{Z}_{2} \otimes \mathbb{Z}_{2}$, and $S$-group has the center $\mathbb{Z}_{4}$. 
Table 2. Finite group structure of the real Clifford algebras.

\begin{tabular}{|c|c|c|c|c|c|c|c|c|c|}
\hline & $\mathrm{p}$ & 1 & 2 & 3 & 4 & 5 & 6 & 7 & $\cdots$ \\
\hline q & & & & & & & & & \\
\hline 0 & $N_{1}$ & $\Omega_{0}$ & $N_{1}$ & $S_{1}$ & $N_{4}$ & $\Omega_{4}$ & $N_{6}$ & $S_{3}$ & $\ldots$ \\
\hline 1 & $S_{0}$ & $N_{1}$ & $\Omega_{1}$ & $N_{3}$ & $S_{2}$ & $N_{6}$ & $\Omega_{6}$ & $N_{8}$ & $\cdots$ \\
\hline 2 & $N_{2}$ & $S_{1}$ & $N_{3}$ & $\Omega_{3}$ & $N_{5}$ & $S_{3}$ & $N_{8}$ & $\Omega_{8}$ & $\cdots$ \\
\hline 3 & $\Omega_{2}$ & $N_{4}$ & $S_{2}$ & $N_{5}$ & $\Omega_{5}$ & $N_{7}$ & $S_{4}$ & $N_{10}$ & . \\
\hline 4 & $N_{4}$ & $\Omega_{4}$ & $N_{6}$ & $S_{3}$ & $N_{7}$ & $\Omega_{7}$ & $N_{9}$ & $S_{5}$ & $\cdots$ \\
\hline 5 & $S_{2}$ & $N_{6}$ & $\Omega_{6}$ & $N_{8}$ & $S_{4}$ & $N_{9}$ & $\Omega_{9}$ & $N_{11}$ & 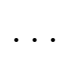 \\
\hline 6 & $N_{5}$ & $S_{3}$ & $N_{8}$ & $\Omega_{8}$ & $N_{10}$ & $S_{5}$ & $N_{11}$ & $\Omega_{11}$ & 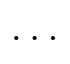 \\
\hline 7 & $\Omega_{5}$ & $N_{7}$ & $S_{4}$ & $N_{10}$ & $\Omega_{10}$ & $N_{12}$ & $S_{6}$ & $N_{13}$ & $\ldots$ \\
\hline : & . & $\vdots$ & $\vdots$ & & & . & . & $\vdots$ & \\
\hline
\end{tabular}

The following Theorem presents a key result in the group structure of $C \ell_{p, q}$.

Theorem 2 (Salingaros [70]). The factor group $G(p, q) / \mathbf{Z}(p, q)$ is the Abelian group $\left(\mathbb{Z}_{2}\right)^{\otimes 2 k}=\mathbb{Z}_{2} \otimes \mathbb{Z}_{2} \otimes \cdots \otimes \mathbb{Z}_{2}$ (2k times):

$$
\frac{G(p, q)}{\mathbf{Z}(p, q)}: \quad \frac{N_{2 k-1}}{\mathbb{Z}_{2}} \simeq \frac{N_{2 k}}{\mathbb{Z}_{2}} \simeq \frac{\Omega_{2 k-1}}{\mathbb{Z}_{2} \otimes \mathbb{Z}_{2}} \simeq \frac{\Omega_{2 k}}{\mathbb{Z}_{2} \otimes \mathbb{Z}_{2}} \simeq \frac{S_{k}}{\mathbb{Z}_{4}} \simeq\left(\mathbb{Z}_{2}\right)^{\otimes 2 k}
$$

This Theorem allows one to identify the Salingaros groups with extraspecial groups [70, 72, 13. As known, a finite group $G$ is called an extraspecial 2group if $G$ is of order $2^{n}$ and $G / \mathbf{Z}(G)$ is the Abelian group $\mathbb{Z}_{2} \otimes \cdots \otimes \mathbb{Z}_{2}(n-1$ times). Further, if $G$ is the extraspecial 2 -group of order $2^{2 k+1}$, then

$$
\begin{aligned}
& G \simeq D_{4} \circ \cdots \circ D_{4} \quad(k \text { times }), \text { or } \\
& G \simeq Q_{4} \circ D_{4} \circ \cdots \circ D_{4} \quad(k-1 \text { times })
\end{aligned}
$$

where o means a central product of two groups: that is, the direct product with centers identified. For example, the direct product $Q_{4} \otimes D_{4}$ has the resulting group of order $8 \times 8=64$, and its center is the direct product of the two individual centers and is equal to $\mathbb{Z}_{2} \otimes \mathbb{Z}_{2}$. In contrast, the central product $Q_{4} \circ D_{4}$ amalgamates the $\mathbb{Z}_{2}$ center of $Q_{4}$ with the $\mathbb{Z}_{2}$ of $D_{4}$ to give the center of $Q_{4} \circ D_{4}$ as $\mathbb{Z}_{2}$. Therefore, the central product $Q_{4} \circ D_{4}$ is of order 32 . In the case where one center is a subgroup of the other center, they both amalgamate into the larger center. 
In terms of the extraspecial groups all the Salingaros groups take the form:

$$
\begin{aligned}
N_{2 k-1} & \simeq\left(N_{1}\right)^{\circ k}, \\
N_{2 k} & \simeq N_{2} \circ\left(N_{1}\right)^{\circ(k-1)}, \\
\Omega_{2 k-1} & \simeq N_{2 k-1} \circ\left(\mathbb{Z}_{2} \otimes \mathbb{Z}_{2}\right), \\
\Omega_{2 k} & \simeq N_{2 k} \circ\left(\mathbb{Z}_{2} \otimes \mathbb{Z}_{2}\right), \\
S_{k} & \simeq N_{2 k-1} \circ \mathbb{Z}_{4} \simeq N_{2 k} \circ \mathbb{Z}_{4} .
\end{aligned}
$$

It should be noted here that a comprehensive consideration of the finite groups (13) over a Galouis field $G F(2)=\mathbb{F}_{2}=\{0,1\}$ was given by Shaw [73].

In the following sections we will consider finite groups that arise from the fundamental automorphisms of the Clifford algebras. It will be shown that these groups (both Abelian and non-Abelian) are the subgroups (except some extended automorphism groups) of the Salingaros groups. Moreover, it will be shown also that the finite groups of automorphisms, $\operatorname{Aut}\left(C \ell_{p, q}\right)$ and $\operatorname{Ext}\left(C \ell_{p, q}\right)$, form a natural basis for description of the discrete symmetries in quantum field theory.

\subsection{Finite groups and fundamental automorphisms}

In virtue of the Wedderburn-Artin theorem the all fundamental automorphisms of $\mathrm{Cl}$ are transferred to the matrix algebra. Matrix (spinor) representations of the fundamental automorphisms of $\mathbb{C}_{n}$ were first obtained by Rashevskii in 1955 [65]: 1) Involution: $A^{\star}=W^{*} W^{-1}$, where $W$ is a matrix of the automorphism $\star$ (matrix representation of the volume element $\omega$ ); 2) Reversion: $\widetilde{A}=E A^{\top} E^{-1}$, where $E$ is a matrix of the antiautomorphism $\sim$ satisfying the conditions $\mathcal{E}_{i} \mathrm{E}-\mathrm{E} \mathcal{E}_{i}^{\top}=0$ and $\mathrm{E}^{\top}=(-1)^{\frac{m(m-1)}{2}} \mathrm{E}$, here $\mathcal{E}_{i}=\gamma\left(\mathbf{e}_{i}\right)$ are matrix representations of the units of the algebra $C \ell$; 3) Conjugation: $\widetilde{\mathrm{A}^{\star}}=\mathrm{CA}^{\top} \mathrm{C}^{-1}$, where $\mathrm{C}=\mathrm{EW}^{\top}$ is a matrix of the antiautomorphism $\widetilde{\star}$ satisfying the conditions $C \mathcal{E}_{i}^{\top}+\mathcal{E}_{i} \mathrm{C}=0$ and $\mathrm{C}^{\top}=(-1)^{\frac{m(m+1)}{2}} \mathrm{C}$.

A spinor representation of the pseudoautomorphism $\mathcal{A} \rightarrow \overline{\mathcal{A}}$ of the algebra $\mathbb{C}_{n}$ when $n \equiv 0(\bmod 2)$ is defined as follows. In the spinor representation the every element $\mathcal{A} \in \mathbb{C}_{n}$ should be represented by some matrix $\mathrm{A}$, and the pseudoautomorphism (10) takes a form of the pseudoautomorphism of the full matrix algebra $\mathrm{M}_{2^{n / 2}}$ :

$$
\mathrm{A} \longrightarrow \overline{\mathrm{A}} \text {. }
$$

On the other hand, a transformation replacing the matrix $\mathrm{A}$ by the complex

conjugate matrix, $A \rightarrow \dot{A}$, is also some pseudoautomorphism of the algebra $\mathrm{M}_{2^{n / 2}}$. The composition of the two pseudoautomorpisms $\dot{A} \rightarrow \mathrm{A}$ and $\mathrm{A} \rightarrow \overline{\mathrm{A}}$, 
$\dot{\mathrm{A}} \rightarrow \mathrm{A} \rightarrow \overline{\mathrm{A}}$, is an internal automorphism $\dot{\mathrm{A}} \rightarrow \overline{\mathrm{A}}$ of the full matrix algebra $\mathrm{M}_{2^{n / 2}}$ :

$$
\overline{\mathrm{A}}=\Pi \dot{\mathrm{A}} \Pi^{-1},
$$

where $\Pi$ is a matrix of the pseudoautomorphism $\mathcal{A} \rightarrow \overline{\mathcal{A}}$ in the spinor representation. The sufficient condition for definition of the pseudoautomorphism $\mathcal{A} \rightarrow \overline{\mathcal{A}}$ is a choice of the matrix $\Pi$ in such a way that the transformation $\mathrm{A} \rightarrow \Pi \dot{A} \Pi^{-1}$ transfers into itself the matrices $\mathcal{E}_{1}, \ldots, \mathcal{E}_{p}, i \mathcal{E}_{p+1}, \ldots, i \mathcal{E}_{p+q}$ (the matrices of the spinbasis of $C_{p, q}$ ), that is,

$$
\mathcal{E}_{i} \longrightarrow \mathcal{E}_{i}=\Pi \dot{\varepsilon}_{i} \Pi^{-1} \quad(i=1, \ldots, p+q) .
$$

Theorem 3. Let $\mathbb{C}_{n}$ be a complex Clifford algebra for $n \equiv 0(\bmod 2)$ and let $C l_{p, q} \subset \mathbb{C}_{n}$ be its subalgebra with a real division ring $\mathbb{K} \simeq \mathbb{R}$ when $p-q \equiv 0,2$ $(\bmod 8)$ and quaternionic division ring $\mathbb{K} \simeq \mathbb{H}$ when $p-q \equiv 4,6(\bmod 8)$, $n=p+q$. Then in dependence on the division ring structure of the real subalgebra $C_{p, q}$ the matrix $\Pi$ of the pseudoautomorphism $\mathcal{A} \rightarrow \overline{\mathcal{A}}$ has the following form:

1) $\mathbb{K} \simeq \mathbb{R}, p-q \equiv 0,2(\bmod 8)$.

The matrix $\Pi$ for any spinor representation over the ring $\mathbb{K} \simeq \mathbb{R}$ is proportional to the unit matrix.

2) $\mathbb{K} \simeq \mathbb{H}, p-q \equiv 4,6(\bmod 8)$.

$\Pi=\mathcal{E}_{\alpha_{1} \alpha_{2} \cdots \alpha_{a}}$ when $a \equiv 0(\bmod 2)$ and $\Pi=\mathcal{E}_{\beta_{1} \beta_{2} \cdots \beta_{b}}$ when $b \equiv 1(\bmod 2)$, where a complex matrices $\mathcal{E}_{\alpha_{t}}$ and $b$ real matrices $\mathcal{E}_{\beta_{s}}$ form a basis of the spinor representation of the algebra $C \ell_{p, q}$ over the ring $\mathbb{K} \simeq \mathbb{H}, a+b=p+q, 0<t \leq$ $a, 0<s \leq b$. At this point,

$$
\begin{aligned}
& \text { Пா }=\quad \text { । if } a, b \equiv 0,1 \quad(\bmod 4) \text {, } \\
& \text { Пா }=-\mathrm{l} \text { if } a, b \equiv 2,3(\bmod 4) \text {, }
\end{aligned}
$$

where I is the unit matrix.

Proof. The algebra $\mathbb{C}_{n}(n \equiv 0(\bmod 2), n=p+q)$ in virtue of $\mathbb{C}_{n}=\mathbb{C} \otimes C \ell_{p, q}$ and definition of the division ring $\mathbb{K} \simeq f C \ell_{p, q} f$ ( $f$ is a primitive idempotent of the algebra $\left.C \ell_{p, q}\right)$ has four different real subalgebras: $p-q \equiv 0,2(\bmod 8)$ for the real division $\operatorname{ring} \mathbb{K} \simeq \mathbb{R}$ and $p-q \equiv 4,6(\bmod 8)$ for the quaternionic division $\operatorname{ring} \mathbb{K} \simeq \mathbb{H}$.

1) $\mathbb{K} \simeq \mathbb{R}$.

Since for the types $p-q \equiv 0,2(\bmod 8)$ there is an isomorphism $C \ell_{p, q} \simeq$ 
$\mathrm{M}_{2^{\frac{p+q}{2}}}(\mathbb{R})$ (Wedderburn-Artin Theorem), then all the matrices $\mathcal{E}_{i}$ of the spinbasis of $\ell_{p, q}$ are real and $\dot{\varepsilon}_{i}=\varepsilon_{i}$. Therefore, in this case the condition (17) can be written as follows

$$
\mathcal{E}_{i} \longrightarrow \mathcal{E}_{i}=\Pi \mathcal{E}_{i} \Pi^{-1}
$$

whence $\mathcal{E}_{i} \Pi=\Pi \mathcal{E}_{i}$. Thus, for the algebras $C_{p, q}$ of the types $p-q \equiv 0,2$ $(\bmod 8)$ the matrix $\Pi$ of the pseudoautomorphism $\mathcal{A} \rightarrow \overline{\mathcal{A}}$ commutes with all the matrices $\varepsilon_{i}$. It is easy to see that $\Pi \sim \mathrm{I}$.

2) $\mathbb{K} \simeq \mathbb{H}$.

In turn, for the quaternionic types $p-q \equiv 4,6(\bmod 8)$ there is an isomorphism $C \ell_{p, q} \simeq \mathrm{M}_{2 \frac{p+q}{2}}(\mathbb{H})$. Therefore, among the matrices of the spinbasis of the algebra $C \ell_{p, q}$ there are matrices $\mathcal{E}_{\alpha}$ satisfying the condition $\dot{\varepsilon}_{\alpha}=-\mathcal{E}_{\alpha}$. Let $a$ be a quantity of the complex matrices, then the spinbasis of $C \ell_{p, q}$ is divided into two subsets. The first subset $\left\{\dot{\mathcal{E}}_{\alpha_{t}}=-\varepsilon_{\alpha_{t}}\right\}$ contains complex matrices, $0<t \leq$ $a$, and the second subset $\left\{\dot{\mathcal{E}}_{\beta_{s}}=\mathcal{E}_{\beta_{s}}\right\}$ contains real matrices, $0<s \leq p+q-a$. In accordance with a spinbasis structure of the algebra $C \ell_{p, q} \simeq \mathrm{M}_{2 \frac{p+q}{2}}(\mathbb{H})$ the condition (17) can be written as follows

$$
\mathcal{E}_{\alpha_{t}} \longrightarrow-\varepsilon_{\alpha_{t}}=\Pi \mathcal{E}_{\alpha_{t}} \Pi^{-1}, \quad \mathcal{E}_{\beta_{s}} \longrightarrow \mathcal{E}_{\beta_{s}}=\Pi \mathcal{E}_{\beta_{s}} \Pi^{-1} .
$$

Whence

$$
\mathcal{E}_{\alpha_{t}} \Pi=-\Pi \mathcal{E}_{\alpha_{t}}, \quad \mathcal{\varepsilon}_{\beta_{s}} \Pi=\Pi \mathcal{E}_{\beta_{s}} .
$$

Thus, for the quaternionic types $p-q \equiv 4,6(\bmod 8)$ the matrix $\Pi$ of the pseudoautomorphism $\mathcal{A} \rightarrow \overline{\mathcal{A}}$ anticommutes with a complex part of the spinbasis of $C \ell_{p, q}$ and commutes with a real part of the same spinbasis. From (18) it follows that a structure of the matrix $\Pi$ is analogous to the structure of the matrices $\mathrm{E}$ and $\mathrm{C}$ of the antiautomorphisms $\mathcal{A} \rightarrow \widetilde{\mathcal{A}}$ and $\mathcal{A} \rightarrow \widetilde{\mathcal{A}^{\star}}$, correspondingly (see Theorem $[$ ) , that is, the matrix $\Pi$ of the pseudoautomorphism $\mathcal{A} \rightarrow \overline{\mathcal{A}}$ of the algebra $\mathbb{C}_{n}$ is a product of only complex matrices, or only real matrices of the spinbasis of the subalgebra $C \ell_{p, q}$.

So, let $0<a<p+q$ and let $\Pi=\mathcal{E}_{\alpha_{1} \alpha_{2} \cdots \alpha_{a}}$ be a matrix of $\mathcal{A} \rightarrow \overline{\mathcal{A}}$, then permutation conditions of the matrix $\Pi$ with the matrices $\mathcal{E}_{\beta_{s}}$ of the real part $(0<s \leq p+q-a)$ and with the matrices $\mathcal{E}_{\alpha_{t}}$ of the complex part $(0<t \leq a)$ have the form

$$
\begin{gathered}
\Pi \mathcal{E}_{\beta_{s}}=(-1)^{a} \mathcal{E}_{\beta_{s}} \Pi, \\
\Pi \mathcal{E}_{\alpha_{t}}=(-1)^{a-t} \sigma\left(\alpha_{t}\right) \mathcal{E}_{\alpha_{1} \alpha_{2} \cdots \alpha_{t-1} \alpha_{t+1} \cdots \alpha_{a}}, \\
\mathcal{E}_{\alpha_{t}} \Pi=(-1)^{t-1} \sigma\left(\alpha_{t}\right) \mathcal{E}_{\alpha_{1} \alpha_{2} \cdots \alpha_{t-1} \alpha_{t+1} \cdots \alpha_{a}},
\end{gathered}
$$


that is, when $a \equiv 0(\bmod 2)$ the matrix $\Pi$ commutes with the real part and anticommutes with the complex part of the spinbasis of $C \ell_{p, q}$. Correspondingly, when $a \equiv 1(\bmod 2)$ the matrix $\Pi$ anticommutes with the real part and commutes with the complex part. Further, let $\Pi=\mathcal{E}_{\beta_{1} \beta_{2} \cdots \beta_{p+q-a}}$ be a product of the real matrices, then

$$
\begin{array}{cc}
\Pi \mathcal{E}_{\beta_{s}}= & (-1)^{p+q-a-s} \sigma\left(\beta_{s}\right) \mathcal{E}_{\beta_{1} \beta_{2} \cdots \beta_{s-1} \beta_{s+1} \cdots \beta_{p+q-a}}, \\
\mathcal{E}_{\beta_{s}} \Pi= & (-1)^{s-1} \sigma\left(\beta_{s}\right) \mathcal{E}_{\beta_{1} \beta_{2} \cdots \beta_{s-1} \beta_{s+1} \cdots \beta_{p+q-a}}, \\
\Pi \mathcal{E}_{\alpha_{t}}=(-1)^{p+q-a} \mathcal{E}_{\alpha_{t}} \Pi,
\end{array}
$$

that is, when $p+q-a \equiv 0(\bmod 2)$ the matrix $\Pi$ anticommutes with the real part and commutes with the complex part of the spinbasis of $C \ell_{p, q}$. Correspondingly, when $p+q-a \equiv 1(\bmod 2)$ the matrix $\Pi$ commutes with the real part and anticommutes with the complex part.

The comparison of the conditions (19)-(20) with the condition (18) shows that the matrix $\Pi=\mathcal{E}_{\alpha_{1} \alpha_{2} \cdots \alpha_{a}}$ exists only at $a \equiv 0(\bmod 2)$, that is, $\Pi$ is a product of the complex matrices $\mathcal{E}_{\alpha_{t}}$ of the even number. In turn, a comparison of (21)-(22) with (18) shows that the matrix $\Pi=\mathcal{E}_{\beta_{1} \beta_{2} \cdots \beta_{p+q-a}}$ exists only at $p+q-a \equiv 1(\bmod 2)$, that is, $\Pi$ is a product of the real matrices $\mathcal{E}_{\beta_{s}}$ of the odd number.

Let us calculate now the product $\Pi \dot{\Pi}$. Let $\Pi=\mathcal{E}_{\beta_{1} \beta_{2} \cdots \beta_{p+q-a}}$ be a product of the $p+q-a$ real matrices. Since $\dot{\mathcal{\varepsilon}}_{\beta_{s}}=\mathcal{E}_{\beta_{s}}$, then $\dot{\Pi}=\Pi$ and $\Pi \dot{\Pi}=\Pi^{2}$. Therefore,

$$
\Pi \dot{\Pi}=\left(\mathcal{E}_{\beta_{1} \beta_{2} \cdots \beta_{p+q-a}}\right)^{2}=(-1)^{\frac{(p+q-a)(p+q-a-1)}{2}} \cdot \text { I. }
$$

Further, let $\Pi=\mathcal{E}_{\alpha_{1} \alpha_{2} \cdots \alpha_{a}}$ be a product of the a complex matrices. Then $\dot{\mathcal{E}}_{\alpha_{t}}=-\mathcal{E}_{\alpha_{t}}$ and $\dot{\Pi}=(-1)^{a} \Pi=\Pi$, since $a \equiv 0(\bmod 2)$. Therefore,

$$
\Pi \dot{\Pi}=\left(\mathcal{E}_{\alpha_{1} \alpha_{2} \cdots \alpha_{a}}\right)^{2}=(-1)^{\frac{a(a-1)}{2}} \cdot \text { I. }
$$

Let $p+q-a=b$ be a quantity of the real matrices $\mathcal{E}_{\beta_{s}}$ of the spinbasis of $C_{p, q}$, then $p+q=a+b$. Since $p+q$ is always even number for the quaternionic types $p-q \equiv 4,6(\bmod 8)$, then $a$ and $b$ are simultaneously even or odd numbers. Thus, from (23) and (24) it follows

$$
\Pi \dot{\Pi}=\left\{\begin{aligned}
\mathrm{I}, & \text { if } a, b \equiv 0,1(\bmod 4), \\
-\mathrm{l}, & \text { if } a, b \equiv 2,3(\bmod 4),
\end{aligned}\right.
$$

which required to be proved. 
In the present form of quantum field theory complex fields correspond to charged particles. Thus, the extraction of the subalgebra $C \ell_{p, q}$ with the real ring $\mathbb{K} \simeq \mathbb{R}$ in $\mathbb{C}_{n}, p-q \equiv 0,2(\bmod 8)$, corresponds to physical fields describing truly neutral particles such as photon and neutral mesons $\left(\pi^{0}, \eta^{0}, \rho^{0}, \omega^{0}, \varphi^{0}, K^{0}\right)$. In turn, the subalgebras $C \ell_{p, q}$ with the ring $\mathbb{K} \simeq \mathbb{H}$, $p-q \equiv 4,6(\bmod 8)$ correspond to charged or neutral fields.

In the paper [78] it has been shown that space reversal $P$, time reversal $T$ and combination $P T$ are correspond respectively to the fundamental automorphisms $\mathcal{A} \rightarrow \mathcal{A}^{\star}, \mathcal{A} \rightarrow \widetilde{\mathcal{A}}$ and $\mathcal{A} \rightarrow \widetilde{\mathcal{A}^{\star}}$. In like manner, charge conjugation $C$ is naturally included into a general scheme by means of a complex conjugation pseudoautomorphism $\mathcal{A} \rightarrow \bar{A}$.

Proposition 1. Let $C_{p, q}(p+q=2 m)$ be a Clifford algebra over the field $\mathbb{F}=\mathbb{R}$ and let $\operatorname{Pin}(p, q)$ be an universal covering of the orthogonal group $O(p, q)=$ $O_{0}(p, q) \odot\{1, P, T, P T\} \simeq O_{0}(p, q) \odot\left(\mathbb{Z}_{2} \otimes \mathbb{Z}_{2}\right)$ of transformations of the space $\mathbb{R}^{p, q}$, where $\{1, P, T, P T\} \simeq \mathbb{Z}_{2} \otimes \mathbb{Z}_{2}$ is a group of discrete transformations of $\mathbb{R}^{p, q}, \mathbb{Z}_{2} \otimes \mathbb{Z}_{2}$ is the Gauss-Klein group. Then there is an isomorphism between the group $\{1, P, T, P T\}$ and an automorphism group $\{\mathrm{Id}, \star, \sim, \widetilde{\star}\}$ of the algebra $C \ell_{p, q}$. In this case, space inversion $P$, time reversal $T$ and combination $P T$ are correspond to the fundamental automorphisms $\mathcal{A} \rightarrow \mathcal{A}^{\star}, \mathcal{A} \rightarrow \widetilde{\mathcal{A}}$ and $\mathcal{A} \rightarrow \widetilde{\mathcal{A}^{\star}}$.

An equivalence of the multiplication tables of the groups $\{1, P, T, P T\}$ and $\operatorname{Aut}(C \ell)=\{\mathrm{Id}, \star, \sim \widetilde{\star}\}$ proves this isomorphism (in virtue of the commutativity $\widetilde{\left(\mathcal{A}^{\star}\right)}=(\widetilde{\mathcal{A}})^{\star}$ and the involution conditions $\left.(\star)^{2}=\left({ }^{\sim}\right)^{2}=\mathrm{Id}\right)$ :

\begin{tabular}{|c||c|c|c|c|}
\hline & Id & $\star$ & $\sim$ & $\widetilde{\star}$ \\
\hline \hline Id & Id & $\star$ & $\sim$ & $\widetilde{\star}$ \\
\hline$\star$ & $\star$ & Id & $\widetilde{\star}$ & $\sim$ \\
\hline$\sim$ & $\sim$ & $\widetilde{I}$ & Id & $\star$ \\
\hline$\widetilde{\star}$ & $\widetilde{\star}$ & $\sim$ & $\star$ & Id \\
\hline
\end{tabular}

$\sim \sim \mid$\begin{tabular}{c||c|c|c|c|}
\hline & 1 & $P$ & $T$ & $P T$ \\
\hline \hline 1 & 1 & $P$ & $T$ & $P T$ \\
\hline$P$ & $P$ & 1 & $P T$ & $T$ \\
\hline$T$ & $T$ & $P T$ & 1 & $P$ \\
\hline$P T$ & $P T$ & $T$ & $P$ & 1 \\
\hline
\end{tabular}

Further, in the case $P^{2}=T^{2}=(P T)^{2}= \pm 1$ and $P T=-T P$ there is an isomorphism between the group $\{1, P, T, P T\}$ and an automorphism group Aut $(C \ell)=\{\mathrm{I}, \mathrm{W}, \mathrm{E}, \mathrm{C}\}$. So, for the Dirac algebra $\mathbb{C}_{4}$ in the canonical $\gamma$-basis there exists a standard (Wigner) representation $P=\gamma_{0}$ and $T=\gamma_{1} \gamma_{3}$ [9], therefore, $\{1, P, T, P T\}=\left\{1, \gamma_{0}, \gamma_{1} \gamma_{3}, \gamma_{0} \gamma_{1} \gamma_{3}\right\}$. On the other hand, in the $\gamma$-basis an automorphism group of $\mathbb{C}_{4}$ has a form Aut $\left(\mathbb{C}_{4}\right)=\{\mathrm{I}, \mathrm{W}, \mathrm{E}, \mathrm{C}\}=\left\{\mathrm{I}, \gamma_{0} \gamma_{1} \gamma_{2} \gamma_{3}, \gamma_{1} \gamma_{3}, \gamma_{0} \gamma_{2}\right\}$. It has been shown 78. 
that $\{1, P, T, P T\}=\left\{1, \gamma_{0}, \gamma_{1} \gamma_{3}, \gamma_{0} \gamma_{1} \gamma_{3}\right\} \simeq \operatorname{Aut}\left(\mathbb{C}_{4}\right) \simeq \mathbb{Z}_{4}$, where $\mathbb{Z}_{4}$ is a complex group with the signature $(+,-,-)$. Generalizations of these results for the Clifford algebras over the fields $\mathbb{F}=\mathbb{C}$ and $\mathbb{F}=\mathbb{R}$ are contained in the following two theorems:

Theorem 4 (78) $)$. Let Aut $=\{\mathrm{I}, \mathrm{W}, \mathrm{E}, \mathrm{C}\}$ be the automorphism group of the algebra $\mathbb{C}_{p+q}(p+q=2 m)$, where $\mathbf{W}=\mathcal{E}_{12 \cdots m m+1 m+2 \cdots p+q}$, and $\mathbf{E}=\mathcal{E}_{12 \cdots m}$, $\mathrm{C}=\mathcal{E}_{m+1 m+2 \cdots p+q}$ if $m \equiv 1(\bmod 2)$, and $\mathrm{E}=\mathcal{E}_{m+1 m+2 \cdots p+q}, \mathrm{C}=\mathcal{E}_{1} \mathcal{E}_{2} \cdots \mathcal{E}_{m}$ if $m \equiv 0(\bmod 2)$. Let Aut Lnd $_{-}$aut the $_{+}$be the automorphism groups, in which the all elements correspondingly commute $(m \equiv 0(\bmod 2))$ and anticommute $(m \equiv 1(\bmod 2))$. Then over the field $\mathbb{F}=\mathbb{C}$ there are only two non-isomorphic groups: Aut $-\simeq \mathbb{Z}_{2} \otimes \mathbb{Z}_{2}$ for the signature $(+,+,+)$ if $n \equiv 0,1$ $(\bmod 4)$ and Aut $_{+} \simeq Q_{4} / \mathbb{Z}_{2}$ for the signature $(-,-,-)$ if $n \equiv 2,3(\bmod 4)$.

Theorem 5 ([79]). Let $C_{p, q}$ be a Clifford algebra over a field $\mathbb{F}=\mathbb{R}$ and let Aut $\left(C_{p, q}\right)=\{\mathrm{I}, \mathrm{W}, \mathrm{E}, \mathrm{C}\}$ be a group of fundamental automorphisms of the algebra $C_{p, q}$. Then for eight types of the algebras $C_{p, q}$ there exist, depending upon a division ring structure of $\mathrm{C}_{p, q}$, following isomorphisms between finite groups and groups Aut $\left(C_{p, q}\right)$ with different signatures $(a, b, c)$, where $a, b, c \in\{-,+\}$ :

1) $\mathbb{K} \simeq \mathbb{R}$, types $p-q \equiv 0,2(\bmod 8)$.

If $\mathrm{E}=\mathcal{E}_{p+1 p+2 \cdots p+q}$ and $\mathrm{C}=\mathcal{E}_{12 \cdots p}$, then Abelian groups Aut $\left(C \ell_{p, q}\right) \simeq \mathbb{Z}_{2} \otimes \mathbb{Z}_{2}$ with the signature $(+,+,+)$ and Aut $_{-}\left(C_{p, q}\right) \simeq \mathbb{Z}_{4}$ with the signature $(+,-,-)$ exist at $p, q \equiv 0(\bmod 4)$ and $p, q \equiv 2(\bmod 4)$, respectively, for the type $p-q \equiv 0(\bmod 8)$, and also Abelian groups Aut $\left(C_{p, q}\right) \simeq \mathbb{Z}_{4}$ with the signature $(-,-,+)$ and Aut $_{-}\left(C_{p, q}\right) \simeq \mathbb{Z}_{4}$ with the signature $(-,+,-)$ exist at $p \equiv 0(\bmod 4), q \equiv 2(\bmod 4)$ and $p \equiv 2(\bmod 4), q \equiv 0(\bmod 4)$ for the type $p-q \equiv 2(\bmod 8)$, respectively.

If $\mathrm{E}=\mathcal{E}_{12 \cdots p}$ and $\mathrm{C}=\mathcal{E}_{p+1 p+2 \cdots p+q}$, then non-Abelian groups $\operatorname{Aut}_{+}\left(C_{p, q}\right) \simeq$ $D_{4} / \mathbb{Z}_{2}$ with the signature $(+,-,+)$ and $\operatorname{Aut}_{+}\left(C \ell_{p, q}\right) \simeq D_{4} / \mathbb{Z}_{2}$ with the signature $(+,+,-)$ exist at $p, q \equiv 3(\bmod 4)$ and $p, q \equiv 1(\bmod 4)$, respectively, for the type $p-q \equiv 0(\bmod 8)$, and also non-Abelian groups Aut $+\left(C \ell_{p, q}\right) \simeq Q_{4} / \mathbb{Z}_{2}$ with $(-,-,-)$ and Aut $_{+}\left(C_{p, q}\right) \simeq D_{4} / \mathbb{Z}_{2}$ with $(-,+,+)$ exist at $p \equiv 3$ $(\bmod 4), q \equiv 1(\bmod 4)$ and $p \equiv 1(\bmod 4), q \equiv 3(\bmod 4)$ for the type $p-q \equiv 2(\bmod 8)$, respectively.

2) $\mathbb{K} \simeq \mathbb{H}$, types $p-q \equiv 4,6(\bmod 8)$.

If $\mathrm{E}=\mathcal{E}_{j_{1} j_{2} \cdots j_{k}}$ is a product of $k$ skewsymmetric matrices (among which $l$ matrices have a square $+\mathrm{I}$ and $t$ matrices have a square $-\mathrm{I})$ and $\mathrm{C}=\mathcal{E}_{i_{1} i_{2} \cdots i_{p+q-k}}$ is a product of $p+q-k$ symmetric matrices (among which $h$ matrices have a square $+\mathrm{I}$ and $g$ have a square $-\mathrm{I})$, then at $k \equiv 0(\bmod 2)$ for the type $p-q \equiv 4(\bmod 8)$ there exist Abelian groups Aut $\left(C \ell_{p, q}\right) \simeq \mathbb{Z}_{2} \otimes \mathbb{Z}_{2}$ with 
$(+,+,+)$ and Aut $_{-}\left(C_{p, q}\right) \simeq \mathbb{Z}_{4}$ with $(+,-,-)$ if $l-t, h-g \equiv 0,1,4,5(\bmod 8)$ and $l-t, h-g \equiv 2,3,6,7(\bmod 8)$, respectively. And also at $k \equiv 0(\bmod 2)$ for the type $p-q \equiv 6(\bmod 8)$ there exist Aut $-\left(C \ell_{p, q}\right) \simeq \mathbb{Z}_{4}$ with $(-,+,-)$ and Aut $_{-}\left(C_{p, q}\right) \simeq \mathbb{Z}_{4}$ with $(-,-,+)$ if $l-t \equiv 0,1,4,5(\bmod 8), h-g \equiv 2,3,6,7$ $(\bmod 8)$ and $l-t \equiv 2,3,6,7(\bmod 8), h-g \equiv 0,1,4,5(\bmod 8)$,respectively.

Inversely, if $\mathrm{E}=\mathcal{E}_{i_{1} i_{2} \cdots i_{p+q-k}}$ is a product of $p+q-k$ symmetric matrices and $\mathrm{C}=\mathcal{E}_{j_{1} j_{2} \cdots j_{k}}$ is a product of $k$ skewsymmetric matrices, then at $k \equiv 1(\bmod 2)$ for the type $p-q \equiv 4(\bmod 8)$ there exist non-Abelian groups Aut $_{+}\left(C \ell_{p, q}\right) \simeq D_{4} / \mathbb{Z}_{2}$ with $(+,-,+)$ and Aut $_{+}\left(C \ell_{p, q}\right) \simeq D_{4} / \mathbb{Z}_{2}$ with $(+,+,-)$ if $h-g \equiv 2,3,6,7(\bmod 8), l-t \equiv 0,1,4,5(\bmod 8)$ and $h-g \equiv 0,1,4,5$ $(\bmod 8), l-t \equiv 2,3,6,7(\bmod 8)$, respectively. And also at $k \equiv 1(\bmod 2)$ for the type $p-q \equiv 6(\bmod 8)$ there exist Aut $_{+}\left(C_{p, q}\right) \simeq Q_{4} / \mathbb{Z}_{2}$ with $(-,-,-)$ and Aut $_{+}\left(C_{p, q}\right) \simeq D_{4} / \mathbb{Z}_{2}$ with $(-,+,+)$ if $h-g, l-t \equiv 2,3,6,7(\bmod 8)$ and $h-g, l-t \equiv 0,1,4,5(\bmod 8)$, respectively.

3) $\mathbb{K} \simeq \mathbb{R} \oplus \mathbb{R}, \mathbb{K} \simeq \mathbb{H} \oplus \mathbb{H}$, types $p-q \equiv 1,5(\bmod 8)$.

For the algebras $C_{0, q}$ of the types $p-q \equiv 1,5(\bmod 8)$ there exist Abelian automorphism groups with the signatures $(-,-,+),(-,+,-)$ and non-Abelian automorphism groups with the signatures $(-,-,-),(-,+,+)$. Correspondingly, for the algebras $C_{p, 0}$ of the types $p-q \equiv 1,5(\bmod 8)$ there exist Abelian groups with $(+,+,+),(+,-,-)$ and non-Abelian groups with $(+,-,+),(+,+,-)$. In general case for $C_{p, q}$, the types $p-q \equiv 1,5(\bmod 8)$ admit all eight automorphism groups.

4) $\mathbb{K}=\mathbb{C}$, types $p-q \equiv 3,7(\bmod 8)$.

The types $p-q \equiv 3,7(\bmod 8)$ admit the Abelian group Aut_ $\left(C \ell_{p, q}\right) \simeq \mathbb{Z}_{2} \otimes \mathbb{Z}_{2}$ with the signature $(+,+,+)$ if $p \equiv 0(\bmod 2)$ and $q \equiv 1(\bmod 2)$, and also non-Abelian group Aut $_{+}\left(C_{p, q}\right) \simeq Q_{4} / \mathbb{Z}_{2}$ with the signature $(-,-,-)$ if $p \equiv 1$ $(\bmod 2)$ and $q \equiv 0(\bmod 2)$.

\section{$3 \quad$ Extended automorphism groups}

An introduction of the pseudoautomorphism $\mathcal{A} \rightarrow \overline{\mathcal{A}}$ allows us to extend the automorphism set of the complex Clifford algebra $\mathbb{C}_{n}$. Namely, we add to the four fundamental automorphisms $\mathcal{A} \rightarrow \mathcal{A}, \mathcal{A} \rightarrow \mathcal{A}^{\star}, \mathcal{A} \rightarrow \widetilde{\mathcal{A}}, \mathcal{A} \rightarrow \widetilde{\mathcal{A}^{\star}}$ the pseudoautomorphism $\mathcal{A} \rightarrow \overline{\mathcal{A}}$ and following three combinations:

1) A pseudoautomorphism $\mathcal{A} \rightarrow \overline{\mathcal{A}^{\star}}$. This transformation is a composition of the pseudoautomorphism $\mathcal{A} \rightarrow \overline{\mathcal{A}}$ with the automorphism $\mathcal{A} \rightarrow \mathcal{A}^{\star}$.

2) A pseudoantiautomorphism $\mathcal{A} \rightarrow \overline{\widetilde{\mathcal{A}}}$. This transformation is a composition of $\mathcal{A} \rightarrow \overline{\mathcal{A}}$ with the antiautomorphism $\mathcal{A} \rightarrow \widetilde{\mathcal{A}}$. 
3) A pseudoantiautomorphism $\mathcal{A} \rightarrow \overline{\widetilde{\mathcal{A}^{\star}}}$ (a composition of $\mathcal{A} \rightarrow \overline{\mathcal{A}}$ with the antiautomorphism $\mathcal{A} \rightarrow \widetilde{\mathcal{A}^{\star}}$.

Thus, we obtain an automorphism set of $\mathbb{C}_{n}$ consisting of the eight transformations. Let us show that the set $\{\mathrm{Id}, \star, \sim \widetilde{\star},-\bar{\star}, \bar{\sim}, \overline{\widetilde{\star}}\}$ forms a finite group of order 8 and let us give a physical interpretation of this group.

Proposition 2. Let $\mathbb{C}_{n}$ be a Clifford algebra over the field $\mathbb{F}=\mathbb{C}$ and let $\operatorname{Ext}\left(\mathbb{C}_{n}\right)=\{\mathrm{Id}, \star, \sim, \widetilde{\star},-\bar{\star}, \bar{\sim}, \overline{\widetilde{\star}}\}$ be an extended automorphism group of the algebra $\mathbb{C}_{n}$. Then there is an isomorphism between $\operatorname{Ext}\left(\mathbb{C}_{n}\right)$ and the full $C P T$-group of the discrete transformations, $\operatorname{Ext}\left(\mathbb{C}_{n}\right) \simeq$ $\{1, P, T, P T, C, C P, C T, C P T\} \simeq \mathbb{Z}_{2} \otimes \mathbb{Z}_{2} \otimes \mathbb{Z}_{2}$. In this case, space inversion $P$, time reversal $T$, full reflection $P T$, charge conjugation $C$, transformations $C P, C T$ and the full CPT-transformation correspond to the automorphism $\mathcal{A} \rightarrow \mathcal{A}^{\star}$, antiautomorphisms $\mathcal{A} \rightarrow \widetilde{\mathcal{A}}, \mathcal{A} \rightarrow \widetilde{\mathcal{A}^{\star}}$, pseudoautomorphisms $\mathcal{A} \rightarrow \overline{\mathcal{A}}$, $\mathcal{A} \rightarrow \overline{\mathcal{A}^{\star}}$, pseudoantiautomorphisms $\mathcal{A} \rightarrow \overline{\widetilde{\mathcal{A}}}$ and $\mathcal{A} \rightarrow \overline{\widetilde{\mathcal{A}^{\star}}}$, respectively .

Proof. The group $\{1, P, T, P T, C, C P, C T, C P T\}$ at the conditions $P^{2}=$ $T^{2}=(P T)^{2}=C^{2}=(C P)^{2}=(C T)^{2}=(C P T)^{2}=1$ and commutativity of all the elements forms an Abelian group of order 8 , which is isomorphic to a cyclic group $\mathbb{Z}_{2} \otimes \mathbb{Z}_{2} \otimes \mathbb{Z}_{2}$. The multiplication table of this group has a form

\begin{tabular}{|c||c|c|c|c|c|c|c|c|}
\hline & 1 & $P$ & $T$ & $P T$ & $C$ & $C P$ & $C T$ & $C P T$ \\
\hline \hline 1 & 1 & $P$ & $T$ & $P T$ & $C$ & $C P$ & $C T$ & $C P T$ \\
\hline$P$ & $P$ & 1 & $P T$ & $T$ & $C P$ & $C$ & $C P T$ & $C T$ \\
\hline$T$ & $T$ & $P T$ & 1 & $P$ & $C T$ & $C P T$ & $C$ & $C P$ \\
\hline$P T$ & $P T$ & $T$ & $P$ & 1 & $C P T$ & $C T$ & $C P$ & $C$ \\
\hline$C$ & $C$ & $C P$ & $C T$ & $C P T$ & 1 & $P$ & $T$ & $P T$ \\
\hline$C P$ & $C P$ & $C$ & $C P T$ & $C T$ & $P$ & 1 & $P T$ & $T$ \\
\hline$C T$ & $C T$ & $C P T$ & $C$ & $C P$ & $T$ & $P T$ & 1 & $P$ \\
\hline$C P T$ & $C P T$ & $C T$ & $C P$ & $C$ & $P T$ & $T$ & $P$ & 1 \\
\hline
\end{tabular}

In turn, for the extended automorphism group $\{\operatorname{Id}, \star, \sim, \widetilde{\star},-\bar{\star}, \bar{\sim}, \overline{\widetilde{\star}}\}$ in virtue of commutativity $\widetilde{\left(\mathcal{A}^{\star}\right)}=(\widetilde{\mathcal{A}})^{\star}, \overline{\left(\mathcal{A}^{\star}\right)}=(\overline{\mathcal{A}})^{\star}, \overline{(\widetilde{\mathcal{A}})}=\overline{(\overline{\mathcal{A}})}, \overline{(\widetilde{\mathcal{A}})^{\star}}=$ $\widetilde{(\widetilde{\mathcal{A}})^{\star}}$ and an involution property $\star \star=\sim \sim=--$ Id we have a following multiplication table 


\begin{tabular}{|c|c|c|c|c|c|c|c|c|}
\hline & Id & $\star$ & $\sim$ & $\widetilde{\star}$ & - & $\bar{\star}$ & $\bar{\sim}$ & $\overline{\widetilde{\star}}$ \\
\hline Id & Id & $\star$ & $\sim$ & $\tilde{\star}$ & - & $\mp$ & $\bar{\sim}$ & $\overline{\widetilde{\star}}$ \\
\hline$\star$ & $\star$ & Id & $\tilde{\star}$ & $\sim$ & $\bar{\star}$ & $\overline{-}$ & $\overline{\widetilde{\widetilde{\star}}}$ & $\bar{\sim}$ \\
\hline$\sim$ & $\sim$ & $\bar{\star}$ & Id & $\star$ & $\bar{\sim}$ & $\overline{\widetilde{\star}}$ & - & $\bar{\star}$ \\
\hline$\widetilde{\star}$ & $\widetilde{\star}$ & $\sim$ & $\star$ & Id & $\overline{\widetilde{\star}}$ & $\bar{\sim}$ & $\bar{\star}$ & - \\
\hline- & - & $\bar{\star}$ & $\bar{\sim}$ & $\overline{\widetilde{\star}}$ & Id & $\star$ & $\sim$ & $\widetilde{\star}$ \\
\hline $\bar{\star}$ & $\mp$ & - & $\overline{\widetilde{\star}}$ & $\bar{\sim}$ & $\star$ & Id & $\widetilde{\star}$ & $\sim$ \\
\hline $\bar{\sim}$ & $\bar{\sim}$ & $\overline{\widetilde{\star}}$ & - & $\bar{\star}$ & $\sim$ & $\widetilde{\star}$ & Id & $\star$ \\
\hline$\overline{\widetilde{\star}}$ & $\overline{\widetilde{\star}}$ & $\bar{\sim}$ & $\bar{\star}$ & - & $\widetilde{\star}$ & $\sim$ & $\star$ & Id \\
\hline
\end{tabular}

The identity of multiplication tables proves the group isomorphism

$$
\begin{aligned}
& \{1, P, T, P T, C, C P, C T, C P T\} \simeq \\
& \{\mathrm{Id}, \star, \sim, \widetilde{\star},-\bar{\star}, \bar{\sim}, \overline{\bar{\star}}\} \simeq \mathbb{Z}_{2} \otimes \mathbb{Z}_{2} \otimes \mathbb{Z}_{2} .
\end{aligned}
$$

Further, in the case of $P^{2}=T^{2}=\ldots=(C P T)^{2}= \pm 1$ and anticommutativity of the elements we have an isomorphism between the $C P T$-group and a group $\operatorname{Ext}\left(\mathbb{C}_{n}\right)$. The elements of $\operatorname{Ext}\left(\mathbb{C}_{n}\right)$ are spinor representations of the automorphisms of the algebra $\mathbb{C}_{n}$. As mentioned previously, the Wedderburn-Artin Theorem allows us to define any spinor representaions for the automorphisms of $\mathbb{C}_{n}$.

\subsection{Pseudoautomorphism $\mathcal{A} \rightarrow \overline{\mathcal{A}^{\star}}$}

Let us find a spinor representation of the pseudoautomorphism $\mathcal{A} \rightarrow \overline{\mathcal{A}^{\star}}$. The transformation $\mathcal{A} \rightarrow \overline{\mathcal{A}^{\star}}$ is a composition of the pseudoautomorphism $\mathcal{A} \rightarrow \overline{\mathcal{A}}$ and the automorphism $\mathcal{A} \rightarrow \mathcal{A}^{\star}$. Under action of $\mathcal{A} \rightarrow \mathcal{A}^{\star}$ we have $\mathbf{e}_{i} \rightarrow-\mathbf{e}_{i}$, where $\mathbf{e}_{i}$ are the units of $C \ell_{p, q}$. In turn, under action of $\mathcal{A} \rightarrow$ $\overline{\mathcal{A}}$ the units $\mathbf{e}_{i}$ remain unaltered, $\mathbf{e}_{i} \rightarrow \mathbf{e}_{i}$. Therefore, under action of the pseudoautomorphism $\mathcal{A} \rightarrow \overline{\mathcal{A}^{\star}}$ we obtain $\mathbf{e}_{i} \rightarrow-\mathbf{e}_{i}$.

As it shown previously, the transformations $\mathcal{A} \rightarrow \mathcal{A}^{\star}$ and $\mathcal{A} \rightarrow \overline{\mathcal{A}}$ in the spinor representation are defined by the expressions $A^{\star}=$ WAW $^{-1}$ and $\overline{\mathrm{A}}=\Pi \dot{A} \Pi^{-1}$. The order of the composition of these transformations is not important $\left(\overline{\mathcal{A}^{\star}}=(\overline{\mathcal{A}})^{\star}=\overline{\left(\mathcal{A}^{\star}\right)}\right)$. Indeed, if $\mathrm{W}$ is a real matrix, then

$$
\overline{\mathrm{A}^{\star}}=\mathrm{W} \Pi \dot{\mathrm{A}} \Pi^{-1} \mathrm{~W}^{-1}=\Pi\left(\mathrm{WAW}^{-1}\right)^{\cdot} \Pi^{-1},
$$


or

$$
\overline{\mathrm{A}^{\star}}=(\mathrm{W} \Pi) \dot{\mathrm{A}}(\mathrm{W} \Pi)^{-1}=(\Pi \mathrm{W}) \dot{\mathrm{A}}(\Pi \mathrm{W})^{-1} .
$$

Otherwise, we have $\overline{\left(A^{\star}\right)}=\Pi \dot{W} \dot{A} \dot{W}^{-1} \Pi^{-1}$. Let us assume that $W$ is a complex matrix, then $\dot{\mathrm{W}}=-\mathrm{W}$ and, therefore, $\overline{\left(\mathrm{A}^{\star}\right)}=\Pi(-\mathrm{W}) \dot{\mathrm{A}}\left(-\mathrm{W}^{-1}\right) \Pi^{-1}=$ (ПW) $\dot{\mathrm{A}}(\Pi \mathrm{W})^{-1}$. Thus, the relation (25) is always fulfilled.

Let $\mathrm{K}=\Pi \mathrm{W}$ be a matrix of the pseudoautomorphism $\mathcal{A} \rightarrow \overline{\mathcal{A}^{\star}}$. Then (25) can be written as follows

$$
\overline{\mathrm{A}^{\star}}=\mathrm{K} \dot{\mathrm{A}} \mathrm{K}^{-1} .
$$

Since under action of the pseudoautomorphism $\mathcal{A} \rightarrow \overline{\mathcal{A}^{\star}}$ we have $\mathbf{e}_{i} \rightarrow-\mathbf{e}_{i}$, in the spinor representation we must demand $\mathcal{E}_{i} \rightarrow-\mathcal{E}_{i}$ also, or

$$
\mathcal{E}_{i} \longrightarrow-\mathcal{E}_{i}=\mathrm{K} \dot{\mathcal{E}}_{i} \mathrm{~K}^{-1} \text {. }
$$

In the case of real subalgebras $C \ell_{p, q}$ with the ring $\mathbb{K} \simeq \mathbb{R}$ we have $\dot{\mathcal{E}}_{i}=\mathcal{E}_{i}$ and the relation (27) takes a form

$$
\mathcal{E}_{i} \longrightarrow-\mathcal{E}_{i}=\mathrm{K}_{i} \mathrm{~K}^{-1}
$$

whence

$$
\varepsilon_{i} \mathrm{~K}=-\mathrm{K} \varepsilon_{i}
$$

that is, the matrix $\mathrm{K}$ is always anticommutes with the matrices of the spinbasis. However, for the ring $\mathbb{K} \simeq \mathbb{R}$ the matrix $\Pi$ of $\mathcal{A} \rightarrow \overline{\mathcal{A}}$ is proportional to the unit matrix, $\Pi \sim \mathrm{I}$ (Theorem 3). Therefore, in this case we have $\mathrm{K} \sim \mathrm{W}$.

In the case of real subalgebras $C_{p, q}$ with the quaternionic ring $\mathbb{K} \simeq \mathbb{H}$ the spinbasis is divided into two parts: a complex part $\left\{\dot{\varepsilon}_{\alpha_{t}}=-\mathcal{E}_{\alpha_{t}}\right\},(0<$ $t \leq a$ ), where $a$ is a number of the complex matrices of the spinbasis, and a real part $\left\{\dot{\mathcal{E}}_{\beta_{s}}=\mathcal{E}_{\beta_{s}}\right\}$, where $p+q-a$ is a number of the real matrices, $(0<s \leq p+q-a)$. Then, in accordance with the spinbasis structure of the algebra $C \ell_{p, q} \simeq \mathrm{M}_{2^{\frac{p+q}{2}}}(\mathbb{H})$, the relation (27) can be written as follows

$$
\mathcal{E}_{\alpha_{t}} \longrightarrow \mathcal{E}_{\alpha_{t}}=\mathrm{K} \varepsilon_{\alpha_{t}} \mathrm{~K}^{-1}, \quad \varepsilon_{\beta_{s}} \longrightarrow-\mathcal{E}_{\beta_{s}}=\mathrm{K} \varepsilon_{\beta_{s}} \mathrm{~K}^{-1} .
$$

Whence

$$
\mathcal{E}_{\alpha_{t}} \mathrm{~K}=\mathrm{K} \varepsilon_{\alpha_{t}}, \quad \varepsilon_{\beta_{s}} \mathrm{~K}=-\mathrm{K} \varepsilon_{\beta_{s}} .
$$

Thus, for the quaternionic types $p-q \equiv 4,6(\bmod 8)$ the matrix $\mathrm{K}$ of the pseudoautomorphism $\mathcal{A} \rightarrow \overline{\mathcal{A}^{\star}}$ commutes with the complex part and anticommutes with the real part of the spinbasis of $C \ell_{p, q}$. Hence it follows that a structure of the matrix $\mathrm{K}$ is analogous to the structure of the matrix $\Pi$ of 
the pseudoautomorphism $\mathcal{A} \rightarrow \overline{\mathcal{A}}$ (see Theorem 3 ), that is, the matrix $\mathrm{K}$ of $\mathcal{A} \rightarrow \overline{\mathcal{A}^{\star}}$ is a product of only complex or only real matrices.

So, let $0<a \leq p+q$ and let $\mathrm{K}=\mathcal{E}_{\alpha_{1} \alpha_{2} \cdots \alpha_{a}}$ be the matrix of the pseudoautomorphism $\mathcal{A} \rightarrow \overline{\mathcal{A}^{\star}}$, then permutation conditions of the matrix $\mathrm{K}$ with the matrices $\mathcal{E}_{\beta_{s}}$ of the real part $(0<s \leq p+q-a)$ and the matrices $\mathcal{E}_{\alpha_{t}}$ of the complex part $(0<t \leq a)$ have the form

$$
\begin{gathered}
\mathrm{K} \mathcal{E}_{\beta_{s}}=(-1)^{a} \mathcal{E}_{\beta_{s}} \mathrm{~K}, \\
\mathrm{~K} \mathcal{E}_{\alpha_{t}}=(-1)^{a-t} \sigma\left(\alpha_{t}\right) \mathcal{E}_{\alpha_{1} \alpha_{2} \cdots \alpha_{t-1} \alpha_{t+1} \cdots \alpha_{a}}, \\
\mathcal{E}_{\alpha_{t}} \mathrm{~K}=(-1)^{t-1} \sigma\left(\alpha_{t}\right) \mathcal{E}_{\alpha_{1} \alpha_{2} \cdots \alpha_{t-1} \alpha_{t+1} \cdots \alpha_{a}},
\end{gathered}
$$

that is, at $a \equiv 0(\bmod 2) \mathrm{K}$ commutes with the real part and anticommutes with the complex part of the spinbasis. Correspondingly, at $a \equiv 1(\bmod 2) \mathrm{K}$ anticommutes with the real and commutes with the complex part. Further, let $\mathrm{K}=\mathcal{E}_{\beta_{1} \beta_{2} \cdots \beta_{p+q-a}}$ be a product of the real matrices of the spinbasis, then

$$
\begin{gathered}
\mathrm{K} \mathcal{E}_{\beta_{s}}=(-1)^{p+q-a-s} \sigma\left(\beta_{s}\right) \mathcal{E}_{\beta_{1} \beta_{2} \cdots \beta_{s-1} \beta_{s+1} \cdots \beta_{p+q-a}}, \\
\mathcal{E}_{\beta_{s}} \mathrm{~K}=(-1)^{s-1} \sigma\left(\beta_{s}\right) \mathcal{E}_{\beta_{1} \beta_{2} \cdots \beta_{s-1} \beta_{s+1} \cdots \beta_{p+q-a}}, \\
\mathrm{~K} \mathcal{E}_{\alpha_{t}}=(-1)^{p+q-a} \mathcal{E}_{\alpha_{t}} \mathrm{~K},
\end{gathered}
$$

that is, at $p+q-a \equiv 0(\bmod 2)$ the matrix $\mathrm{K}$ anticommutes with the real part and commutes with the complex part of the spinbasis. Correspondingly, at $p+q-a \equiv 1(\bmod 2) \mathrm{K}$ commutes with the real and anticommutes with the complex part.

A comparison of the conditions (29)-(30) with (28) shows that the matrix $\mathrm{K}=\mathcal{E}_{\alpha_{1} \alpha_{2} \cdots \alpha_{a}}$ exists only if $a \equiv 1(\bmod 2)$. In turn, a comparison of the conditions (31)-(32) with (28) shows that the matrix $\mathrm{K}=\mathcal{E}_{\beta_{1} \beta_{2} \cdots \beta_{p+q-a}}$ exists only if $p+q-a \equiv 0(\bmod 2)$.

Let us find now squares of the matrix K. In accordance with obtained conditions there exist two possibilities:

1) $\mathrm{K}=\mathcal{E}_{\alpha_{1} \alpha_{2} \cdots \alpha_{a}}, a \equiv 1(\bmod 2)$.

$$
\mathrm{K}^{2}=\left\{\begin{array}{lll}
+\mathrm{I}, & \text { if } a_{+}-a_{-} \equiv 1,5 \quad(\bmod 8) \\
-\mathrm{I}, & \text { if } a_{+}-a_{-} \equiv 3,7 \quad(\bmod 8)
\end{array}\right.
$$

where $a_{+}$and $a_{-}$are numbers of matrices with '+'- and '-'-squares in the product $\mathcal{E}_{\alpha_{1} \alpha_{2} \cdots \alpha_{a}}$.

2) $\mathrm{K}=\mathcal{E}_{\beta_{1} \beta_{2} \cdots \beta_{p+q-a}}, p+q-a \equiv 0(\bmod 2)$. 


$$
\mathrm{K}^{2}=\left\{\begin{array}{lll}
+\mathrm{I}, & \text { if } b_{+}-b_{-} \equiv 0,4 \quad(\bmod 8) \\
-\mathrm{I}, & \text { if } b_{+}-b_{-} \equiv 2,6 \quad(\bmod 8)
\end{array}\right.
$$

where $b_{+}$and $b_{-}$are numbers of matrices with '+'- and '-'-squares in the product $\mathcal{E}_{\beta_{1} \beta_{2} \cdots \beta_{p+q-a}}$, respectively.

\subsection{Pseudoantiautomorphism $\mathcal{A} \rightarrow \overline{\widetilde{\mathcal{A}}}$}

The pseudoantiautomorphism $\mathcal{A} \rightarrow \overline{\widetilde{\mathcal{A}}}$ is the composition of the pseudoautomorphism $\mathcal{A} \rightarrow \overline{\mathcal{A}}$ with the antiautomorphism $\mathcal{A} \rightarrow \widetilde{\mathcal{A}}$. Under action of $\mathcal{A} \rightarrow \widetilde{\mathcal{A}}$ the units $\mathbf{e}_{i}$ remain unaltered, $\mathbf{e}_{i} \rightarrow \mathbf{e}_{i}$. Analogously, under action of $\mathcal{A} \rightarrow \overline{\mathcal{A}}$ we have $\mathbf{e}_{i} \rightarrow \mathbf{e}_{i}$. Therefore, under action of the pseudoantiautomorphism $\mathcal{A} \rightarrow \overline{\widetilde{\mathcal{A}}}$ the units $\mathbf{e}_{i}$ remain unaltered also, $\mathbf{e}_{i} \rightarrow \mathbf{e}_{i}$.

The spinor representations of the transformations $\mathcal{A} \rightarrow \widetilde{\mathcal{A}}$ and $\mathcal{A} \rightarrow \overline{\mathcal{A}}$ are defined by the expressions $\widetilde{\mathrm{A}}=\mathrm{EA}^{\top} \mathrm{E}^{-1}$ and $\overline{\mathrm{A}}=\Pi \dot{\mathrm{A}} \Pi^{-1}$, respectively. Let us find a spinor representation of the transformation $\mathcal{A} \rightarrow \overline{\widetilde{\mathcal{A}}}$. The order of the composition of these transformations is not important, $\overline{\widetilde{\mathcal{A}}}=\widetilde{(\overline{\mathcal{A}})}=\overline{(\widetilde{\mathcal{A}})}$. Indeed,

$$
\overline{\widetilde{A}}=E\left(\Pi \dot{A} \Pi^{-1}\right)^{\top} E^{-1}=\Pi\left(E A^{\top} E^{-1}\right)^{\cdot} \Pi^{-1}
$$

or,

$$
\overline{\widetilde{A}}=(\mathrm{E} \Pi)(\dot{\mathrm{A}})^{\top}(\mathrm{E} \Pi)^{-1}=(\Pi \mathrm{E})\left(\mathrm{A}^{\top}\right)^{\cdot}(\Pi \mathrm{E})^{-1},
$$

since $\Pi^{-1}=\Pi^{\top}$ and $\overline{\widetilde{A}}=\Pi \dot{E}\left(A^{\top}\right)^{\cdot} \dot{E}^{-1} \Pi^{-1}=\Pi E\left(A^{\top}\right)^{\cdot} E^{-1} \Pi^{-1}$ in the case when $\dot{E}=E$ is a real matrix and $\overline{\widetilde{A}}=\Pi \dot{E}\left(A^{\top}\right) \cdot \dot{E}^{-1} \Pi^{-1}=$ $\Pi(-E)\left(A^{\top}\right)^{\cdot}\left(-E^{-1}\right) \Pi^{-1}=\Pi E\left(A^{\top}\right)^{\cdot}(\Pi E)^{-1}$ in the case when $\dot{E}=-E$ is a complex matrix. Let $S=\Pi E$ be a matrix of the pseudoantiautomorphism $\mathcal{A} \rightarrow \overline{\widetilde{\mathcal{A}}}$ in the spinor representation. Then (33) can be rewritten as follows

$$
\overline{\widetilde{A}}=S\left(A^{\top}\right) S^{-1}
$$

Since under action of the transformation $\mathcal{A} \rightarrow \overline{\widetilde{\mathcal{A}}}$ we have $\mathbf{e}_{i} \rightarrow \mathbf{e}_{i}$, in the spinor representation we must demand $\mathcal{E}_{i} \rightarrow \mathcal{E}_{i}$ also, or

$$
\mathcal{E}_{i} \longrightarrow \mathcal{E}_{i}=\mathrm{S} \dot{\mathcal{E}}_{i}^{\top} \mathrm{S}^{-1}
$$


In the case of real subalgebras $C \ell_{p, q}$ with the $\operatorname{ring} \mathbb{K} \simeq \mathbb{R}$ we have $\dot{\mathcal{E}}_{i}=\mathcal{E}_{i}$ and, therefore, the relation (35) takes a form

$$
\mathcal{E}_{i} \longrightarrow \mathcal{E}_{i}=\mathrm{S}_{i}^{\top} \mathrm{S}^{-1}
$$

Let $\left\{\mathcal{E}_{\gamma_{i}}\right\}$ be a set of symmetric matrices $\left(\mathcal{E}_{\gamma_{i}}^{\top}=\mathcal{E}_{\gamma_{i}}\right)$ and let $\left\{\mathcal{E}_{\delta_{j}}\right\}$ be a set of skewsymmetric matrices $\left(\varepsilon_{\delta_{j}}^{\top}=-\varepsilon_{\delta_{j}}\right)$ of the spinbasis of the algebra $C \ell_{p, q}$. Then from the relation (36) it follows

$$
\mathcal{E}_{\gamma_{i}} \longrightarrow \mathcal{E}_{\gamma_{i}}=\mathrm{S} \mathcal{E}_{\gamma_{i}} \mathrm{~S}^{-1}, \quad \mathcal{E}_{\delta_{j}} \longrightarrow \mathcal{E}_{\delta_{j}}=-\mathrm{S} \mathcal{E}_{\delta_{j}} \mathrm{~S}^{-1}
$$

Whence

$$
\mathcal{E}_{\gamma_{i}} \mathrm{~S}=\mathrm{S} \mathcal{E}_{\gamma_{i}}, \quad \mathcal{E}_{\delta_{j}} \mathrm{~S}=-\mathrm{S} \mathcal{E}_{\delta_{j}},
$$

that is, the matrix $S$ of the pseudoantiautomorphism $\mathcal{A} \rightarrow \overline{\widetilde{\mathcal{A}}}$ in the case of $\mathbb{K} \simeq \mathbb{R}$ commutes with the symmetric part and anticommutes with the skewsymmetric part of the spinbasis of $C \ell_{p, q}$. In virtue of Theorem 3 , over the $\operatorname{ring} \mathbb{K} \simeq \mathbb{R}$ the matrix $\Pi$ of the pseudoautomorphism $\mathcal{A} \rightarrow \overline{\mathcal{A}}$ is proportional to the unit matrix, $\Pi \sim$ I. Therefore, in this case we have $S \sim E$ and an explicit form of $\mathbf{S}$ coincides with $\mathrm{E}$.

Further, in case of the quaternionic ring $\mathbb{K} \simeq \mathbb{H}, p-q \equiv 4,6(\bmod 8)$, a spinbasis of $C_{p, q}$ contains both complex matrices $\mathcal{E}_{\alpha_{t}}$ and real matrices $\mathcal{E}_{\beta_{s}}$, among which there are symmetric and skewsymmetric matrices. It is obvious that the sets of complex and real matrices do not coincide with the sets of symmetric and skewsymmetric matrices. Let $\left\{\mathcal{E}_{\alpha_{t}}\right\}$ be a complex part of the spinbasis, then the relation (35) takes a form

$$
\mathcal{E}_{\alpha_{t}} \longrightarrow \mathcal{E}_{\alpha_{t}}=-\mathrm{S} \mathcal{E}_{\alpha_{t}}^{\top} \mathrm{S}^{-1}
$$

Correspondingly, let $\left\{\mathcal{E}_{\alpha_{\gamma}}\right\}$ and $\left\{\mathcal{E}_{\alpha_{\delta}}\right\}$ be the sets of symmetric and skewsymmetric matrices of the complex part. Then the relation (37) can be written as follows

$$
\mathcal{E}_{\alpha_{\gamma}} \longrightarrow \mathcal{E}_{\alpha_{\gamma}}=-\mathrm{S} \mathcal{E}_{\alpha_{\gamma}} \mathrm{S}^{-1}, \quad \mathcal{E}_{\alpha_{\delta}} \longrightarrow \mathcal{E}_{\alpha_{\delta}}=\mathrm{S} \mathcal{E}_{\alpha \delta} \mathrm{S}^{-1}
$$

Whence

$$
\mathcal{E}_{\alpha_{\gamma}} \mathrm{S}=-\mathrm{S} \mathcal{E}_{\alpha_{\gamma}}, \quad \mathcal{E}_{\alpha_{\delta}} \mathrm{S}=\mathrm{S} \mathcal{E}_{\alpha_{\delta}} .
$$

Therefore, the matrix $S$ of the pseudoantiautomorphism $\mathcal{A} \rightarrow \overline{\widetilde{\mathcal{A}}}$ anticommutes with the complex symmetric matrices and commutes with the complex skewsymmetric matrices of the spinbasis of $C \ell_{p, q}$. 
Let us consider now the real part $\left\{\mathcal{E}_{\beta_{s}}\right\}$ of the spinbasis of $C \ell_{p, q}, p-q \equiv 4,6$ $(\bmod 8)$. In this case the relation (35) takes a form

$$
\mathcal{E}_{\beta_{s}} \longrightarrow \mathcal{E}_{\beta_{s}}=\mathrm{S}_{\beta_{s}}^{\mathrm{T}} \mathrm{S}^{-1} \text {. }
$$

Let $\left\{\mathcal{E}_{\beta_{\gamma}}\right\}$ and $\left\{\mathcal{E}_{\beta_{\delta}}\right\}$ be the sets of real symmetric and real skewsymmetric matrices, respectively. Then the relation (39) can be written as follows

$$
\mathcal{E}_{\beta_{\gamma}} \longrightarrow \mathcal{E}_{\beta_{\gamma}}=\mathrm{S} \mathcal{E}_{\beta_{\gamma}} \mathrm{S}^{-1}, \quad \mathcal{E}_{\beta_{\delta}} \longrightarrow \mathcal{E}_{\beta_{\delta}}=-\mathrm{S} \mathcal{E}_{\beta_{\delta}} \mathrm{S}^{-1}
$$

Whence

$$
\mathcal{E}_{\beta_{\gamma}} \mathrm{S}=\mathrm{S} \mathcal{E}_{\beta_{\gamma}}, \quad \mathcal{E}_{\beta_{\delta}} \mathrm{S}=-\mathrm{S} \mathcal{E}_{\beta_{\delta}} .
$$

Thus, the matrix $S$ of the transformation $\mathcal{A} \rightarrow \overline{\widetilde{\mathcal{A}}}$ commutes with the real symmetric matrices and anticommutes with the real skewsymmetric matrices of the spinbasis of $C_{p, q}$.

Let us find now an explicit form of the matrix $S=\Pi E$. In accordance with Theorem 3 for the quaternionic types $p-q \equiv 4,6(\bmod 8)$ the matrix $\Pi$ takes the two different forms: 1) $\Pi=\mathcal{E}_{\alpha_{1} \alpha_{2} \cdots \alpha_{a}}$ is the product of complex matrices at $a \equiv 0(\bmod 2) ; 2) \Pi=\mathcal{E}_{\beta_{1} \beta_{2} \cdots \beta_{b}}$ is the product of real matrices at $b \equiv 1$ $(\bmod 2)$. In turn, for the matrix $\mathrm{E}$ of the antiautomorphism $\mathcal{A} \rightarrow \widetilde{\mathcal{A}}$ over the ring $\mathbb{K} \simeq \mathbb{H}$ (see Theorem 5 ) we have the following two forms: 1 ) $\mathrm{E}=\mathcal{E}_{j_{1} j_{2} \cdots j_{k}}$ is the product of skewsymmetric matrices at $k \equiv 0(\bmod 2) ; 2) \mathrm{E}=\mathcal{E}_{i_{1} i_{2} \cdots i_{p+q-k}}$ is the product of symmetric matrices at $k \equiv 1(\bmod 2)$. Thus, in accordance with definition $S=\prod E$ we have four different products: $S=\mathcal{E}_{\alpha_{1} \alpha_{2} \cdots \alpha_{a}} \mathcal{E}_{j_{1} j_{2} \cdots j_{k}}$, $\mathrm{S}=\mathcal{E}_{\alpha_{1} \alpha_{2} \cdots \alpha_{a}} \mathcal{E}_{i_{1} i_{2} \cdots i_{p+q-k}}, \mathrm{~S}=\mathcal{E}_{\beta_{1} \beta_{2} \cdots \beta_{b}} \mathcal{E}_{j_{1} j_{2} \cdots j_{k}}, \mathrm{~S}=\mathcal{E}_{\beta_{1} \beta_{2} \cdots \beta_{b}} \mathcal{E}_{i_{1} i_{2} \cdots i_{p+q-k}}$. It is obvious that in the given products there are identical matrices.

Let us examine the first product $S=\mathcal{E}_{\alpha_{1} \alpha_{2} \cdots \alpha_{a}} \mathcal{E}_{j_{1 j_{2} \cdots j_{k}}}$. Since in this case $\Pi$ contains all the complex matrices of the spinbasis, among which there are symmetric and skewsymmetric matrices, and E contains all the skewsymmetric matrices of the spinbasis, then $\Pi$ and $E$ contain a quantity of identical matrices (complex skewsymmetric matrices). Let $m$ be a number of the complex skewsymmetric matrices of the spinbasis of the algebra $C \ell_{p, q}, p-q \equiv 4,6$ $(\bmod 8)$. Then the product $\mathrm{S}=\Pi \mathrm{E}$ takes a form

$$
\mathcal{E}_{\alpha_{1} \alpha_{2} \cdots \alpha_{a} j_{1} j_{2} \cdots j_{k}}=(-1)^{\mathcal{N}} \sigma\left(i_{1}\right) \sigma\left(i_{2}\right) \cdots \sigma\left(i_{m}\right) \mathcal{E}_{c_{1} c_{2} \cdots c_{s}}
$$

where the indices $c_{1}, \ldots, c_{s}$ present itself a totality of the indices $\alpha_{1}, \ldots, \alpha_{a}, j_{1}, \ldots, j_{k}$ obtained after removal of the indices occured twice; $\mathcal{N}$ is a number of inversions.

First of all, let us remark that $\mathcal{E}_{c_{1} c_{2} \cdots c_{s}}$ is an even product, since the original product $\Pi E$ is the even product also. Besides, the product $\mathcal{E}_{c_{1} c_{2} \cdots c_{s}}$ contains 
all the complex symmetric matrices and all the real skewsymmetric matrices of the spinbasis.

Let us find now permutation conditions between the matrix $\mathbf{S}=\mathcal{E}_{c_{1} c_{2} \cdots c_{s}}$ and the units of the spinbasis of $C \ell_{p, q}, p-q \equiv 4,6(\bmod 8)$. For the complex symmetric matrices $\mathcal{E}_{\alpha_{\gamma}}$ and complex skewsymmetric matrices $\mathcal{E}_{\alpha_{\delta}}$ we have

$$
\begin{gathered}
\mathrm{S} \mathcal{E}_{\alpha_{\gamma}}=(-1)^{s-\gamma} \mathcal{E}_{c_{1} c_{2} \cdots c_{s-2}}, \\
\mathcal{E}_{\alpha_{\gamma}} \mathrm{S}=(-1)^{\gamma-1} \mathcal{E}_{c_{1} c_{2} \cdots c_{s-2}}, \\
\mathrm{~S} \mathcal{E}_{\alpha_{\delta}}=(-1)^{s} \mathcal{E}_{\alpha_{\delta}} \mathrm{S},
\end{gathered}
$$

that is, the matrix $\mathrm{S}$ always anticommutes with the complex symmetric matrices and always commutes with the complex skewsymmetric matrices, since $s \equiv$ $0(\bmod 2)$. Further, for the real symmetric matrices $\mathcal{E}_{\beta_{\gamma}}$ and real skewsymmetric matrices $\mathcal{E}_{\beta_{\delta}}$ we have

$$
\begin{gathered}
\mathrm{S} \mathcal{E}_{\beta_{\gamma}}=(-1)^{s} \mathcal{E}_{\beta_{\gamma}} \mathrm{S}, \\
\mathrm{S} \mathcal{E}_{\beta_{\delta}}=(-1)^{s-\delta} \mathcal{E}_{c_{1} c_{2} \cdots c_{s-2}}, \\
\mathcal{E}_{\beta_{\delta}} \mathrm{S}=(-1)^{\delta-1} \mathcal{E}_{c_{1} c_{2} \cdots c_{s-2}} .
\end{gathered}
$$

Therefore, $\mathbf{S}$ always commutes with the real symmetric matrices and always anticommutes with the real skewsymmetric matrices.

A comparison of the obtained conditions (41)-(44) with (38) and (40) shows that $\mathrm{S}=\mathcal{E}_{c_{1} c_{2} \cdots c_{s}}$ automatically satisfies the conditions, which define the matrix of the pseudoantiautomorphism $\mathcal{A} \rightarrow \overline{\widetilde{\mathcal{A}}}$.

Let examine the second product $S=\mathcal{E}_{\alpha_{1} \alpha_{2} \cdots \alpha_{a}} \mathcal{E}_{i_{1} i_{2} \cdots i_{p+q-k}}$. In this case $\Pi$ contains all the complex matrices of the spinbasis, among which there are symmetric and skewsymmetric matrices, and E contains all the symmetric matrices of the spinbasis. Thus, $\Pi$ and $E$ contain a quantity of identical complex symmetric matrices. Let $l$ be a number of the complex symmetric matrices, then for the product $S=\Pi E$ we obtain

$$
\mathcal{E}_{\alpha_{1} \alpha_{2} \cdots \alpha_{a} i_{1} i_{2} \cdots i_{p+q-k}}=(-1)^{\mathcal{N}} \sigma\left(i_{1}\right) \sigma\left(i_{2}\right) \cdots \sigma\left(i_{l}\right) \mathcal{E}_{d_{1} d_{2} \cdots d_{g}},
$$

where $d_{1}, \ldots, d_{g}$ present itself a totality of the indices $\alpha_{1}, \ldots, \alpha_{a}, i_{1}, \ldots, i_{p+q-k}$ after removal of the indices occured twice. The product $\mathbf{S}=\mathcal{E}_{d_{1} d_{2} \cdots d_{g}}$ is odd, since the original product $\Pi E$ is odd also. Besides, $\mathcal{E}_{d_{1} d_{2} \cdots d_{g}}$ contains all the complex skewsymmetric matrices and all the real symmetric matrices of the spinbasis. 
Let us find permutation conditions of the matrix $S=\mathcal{E}_{d_{1} d_{2} \cdots d_{g}}$ with the units of the spinbasis of $C \ell_{p, q}$. For the complex part of the spinbasis we have

$$
\begin{gathered}
\mathrm{S} \mathcal{E}_{\alpha_{\gamma}}=(-1)^{g} \mathcal{E}_{\alpha_{\gamma}} \mathrm{S}, \\
\mathrm{S} \mathcal{E}_{\alpha_{\delta}}=(-1)^{g-\delta} \mathcal{E}_{d_{1} d_{2} \cdots d_{g-2}}, \\
\mathcal{E}_{\alpha_{\delta}} \mathrm{S}=(-1)^{\delta-1} \mathcal{E}_{d_{1} d_{2} \cdots d_{g-2}},
\end{gathered}
$$

that is, the matrix $\mathbf{S}$ anticommutes with the complex symmetric matrices and commutes with the complex skewsymmetric matrices, since $g \equiv 1(\bmod 2)$. For the real part of the spinbasis of $C_{p, q}$ we obtain

$$
\begin{gathered}
\mathrm{S} \mathcal{E}_{\beta_{\gamma}}=(-1)^{g-\gamma} \mathcal{E}_{d_{1} d_{2} \cdots d_{g-2}}, \\
\mathcal{E}_{\beta_{\gamma}} \mathrm{S}=(-1)^{\gamma-1} \mathcal{E}_{d_{1} d_{2} \cdots d_{g-2}}, \\
\mathrm{~S} \mathcal{E}_{\beta_{\delta}}=(-1)^{g} \mathcal{E}_{\beta_{\delta}} \mathrm{S} .
\end{gathered}
$$

Therefore, $\mathbf{S}$ commutes with the real symmetric matrices and anticommutes with the real skewsymmetric matrices.

Comparing the obtained conditions (45)-(48) with the conditions (38) and (40) we see that $\mathrm{S}=\mathcal{E}_{d_{1} d_{2} \cdots d_{g}}$ automatically satisfies the conditions which define the matrix of the transformation $\mathcal{A} \rightarrow \overline{\widetilde{\mathcal{A}}}$.

Let us consider now the third product $\mathrm{S}=\mathcal{E}_{\beta_{1} \beta_{2} \cdots \beta_{b}} \mathcal{E}_{j_{1} j_{2} \cdots k}$. In this product the matrix $\Pi$ of the pseudoautomorphism $\mathcal{A} \rightarrow \overline{\mathcal{A}}$ contains all the real matrices of the spinbasis, among which there are symmetric and skewsymmetric matrices, and the matrix $\mathrm{E}$ of $\mathcal{A} \rightarrow \widetilde{\mathcal{A}}$ contains all the skewsymmetric matrices of the spinbasis, among which there are both the real and complex matrices. Therefore, $\mathrm{S}$ contains a quantity of identical real skewsymmetric matrices. Let $u$ be a number of the real skewsymmetric matrices of the spinbasis of the algebra $C \ell_{p, q}, p-q \equiv 4,6(\bmod 8)$, then for the product $\mathrm{S}$ we obtain

$$
\mathcal{E}_{\beta_{1} \beta_{2} \cdots \beta_{b} j_{1} j_{2} \cdots j_{k}}=(-1)^{\mathcal{N}} \sigma\left(i_{1}\right) \sigma\left(i_{2}\right) \cdots \sigma\left(i_{u}\right) \mathcal{E}_{e_{1} e_{2} \cdots e_{h}},
$$

where the indices $e_{1}, e_{2}, \ldots, e_{n}$ present itself a totality of the indices $\beta_{1}, \ldots, \beta_{b}, j_{1}, \ldots, j_{k}$ after removal of the indices occurred twice. The product $S=\mathcal{E}_{e_{1} e_{2} \ldots e_{h}}$ is odd, since the original product $\Pi E$ is odd also. It is easy to see that $\mathcal{E}_{e_{1} e_{2} \cdots e_{h}}$ contains all the real symmetric matrices and all the complex skewsymmetric matrices of the spinbasis. Therefore, the matrix $\mathbf{S}=\mathcal{E}_{e_{1} e_{2} \cdots e_{h}}$ is similar to the matrix $\mathbf{S}=\mathcal{E}_{d_{1} d_{2} \cdots d_{g}}$, and its permutation conditions with the units of the spinbasis of $C_{p, q}$ are equivalent to the relations (45)-(48).

Finally, let us examine the fourth product $S=\mathcal{E}_{\beta_{1} \beta_{2} \cdots \beta_{b}} \mathcal{E}_{i_{1} i_{2} \cdots i_{p+q-k}}$. In turn, this product contains a quantity of identical real symmetric matrices. Let $v$ 
be a number of the real symmetric matrices of the spinbasis of $C \ell_{p, q}$, then

$$
\mathcal{E}_{\beta_{1} \beta_{2} \cdots \beta_{b} i_{1} i_{2} \cdots i_{p+q-k}}=(-1)^{\mathcal{N}} \sigma\left(i_{1}\right) \sigma\left(i_{2}\right) \cdots \sigma\left(i_{v}\right) \mathcal{E}_{f_{1} f_{2} \cdots f_{w}},
$$

where $f_{1}, \ldots, f_{w}$ present itself a totality of the indices $\beta_{1}, \ldots, \beta_{b}, i_{1}, \ldots, i_{p+q-k}$ after removal of the indices occurred twice. The product $S=\mathcal{E}_{f_{1} f_{2} \cdots f_{w}}$ is even, since the original product $\Pi E$ is even also. It is easy to see that $\mathcal{E}_{f_{1} f_{2} \cdots f_{w}}$ contains all the real skewsymmetric matrices and all the complex symmetric matrices of the spinbasis. Therefore, the matrix $S=\mathcal{E}_{f_{1} f_{2} \cdots f_{w}}$ is similar to the matrix $S=\mathcal{E}_{c_{1} c_{2} \cdots c_{s}}$, and its permutation conditions with the units of the spinbasis are equivalent to the relations (41)-(44).

Thus, from the four products we have only two non-equivalent products. The squares of the non-equivalent matrices $S=\mathcal{E}_{c_{1} c_{2} \cdots c_{s}}(s \equiv 0(\bmod 2))$ and $\mathrm{S}=\mathcal{E}_{d_{1} d_{2} \cdots d_{g}}(g \equiv 1(\bmod 2))$ are

$$
\begin{gathered}
\mathrm{S}^{2}=\left(\mathcal{E}_{c_{1} c_{2} \cdots c_{s}}\right)^{2}=\left\{\begin{array}{lll}
+\mathrm{I}, & \text { if } u+l \equiv 0,4 & (\bmod 8), \\
-\mathrm{I}, & \text { if } u+l \equiv 2,6 & (\bmod 8) ;
\end{array}\right. \\
\mathrm{S}^{2}=\left(\mathcal{E}_{d_{1} d_{2} \cdots d_{g}}\right)^{2}=\left\{\begin{array}{lll}
+\mathrm{I}, & \text { if } m+v \equiv 1,5 & (\bmod 8), \\
-\mathrm{I}, & \text { if } m+v \equiv 3,7 & (\bmod 8)
\end{array}\right.
\end{gathered}
$$

\subsection{Pseudoantiautomorphism $\mathcal{A} \rightarrow \overline{\widetilde{\mathcal{A}^{\star}}}$}

The pseudoantiautomorphism $\mathcal{A} \rightarrow \overline{\widetilde{\mathcal{A}^{\star}}}$, that defines the $C P T$-transformation,

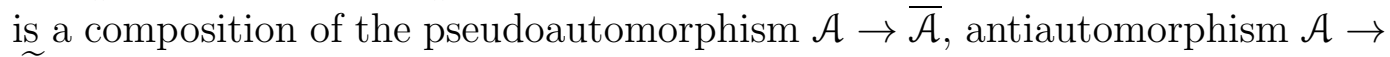
$\widetilde{\mathcal{A}}$ and automorphism $\mathcal{A} \rightarrow \mathcal{A}^{\star}$. Under action of the automorphism $\mathcal{A} \rightarrow \mathcal{A}^{\star}$ the units of $C \ell_{p, q}$ change the sign, $\mathbf{e}_{i} \rightarrow-\mathbf{e}_{i}$. In turn, under action of the transformations $\mathcal{A} \rightarrow \overline{\mathcal{A}}$ and $\mathcal{A} \rightarrow \widetilde{\mathcal{A}}$ the units remain unaltered, $\mathbf{e}_{i} \rightarrow \mathbf{e}_{i}$. Therefore, under action of the pseudoantiautomorphism $\mathcal{A} \rightarrow \overline{\widetilde{\mathcal{A}^{\star}}}$ the units change the sign, $\mathbf{e}_{i} \rightarrow-\mathbf{e}_{i}$.

The spinor representations of the transformations $\mathcal{A} \rightarrow \mathcal{A}^{\star}, \mathcal{A} \rightarrow \widetilde{\mathcal{A}}$ and $\mathcal{A} \rightarrow \overline{\mathcal{A}}$ have the form: $\mathrm{A}^{\star}=\mathrm{WAW}^{-1}, \widetilde{\mathrm{A}}=\mathrm{EA}^{\top} \mathrm{E}^{-1}$ and $\overline{\mathrm{A}}=\Pi \dot{\mathrm{A}} \Pi^{-1}$. Let us find a matrix of the transformation $\mathcal{A} \rightarrow \overline{\widetilde{\mathcal{A}^{\star}}}$. We will consider the pseudoantiautomorphism $\mathcal{A} \rightarrow \overline{\widetilde{\mathcal{A}^{\star}}}$ as a composition of the pseudoautomorphism $\mathcal{A} \rightarrow \overline{\mathcal{A}}$ with the antiautomorphism $\mathcal{A} \rightarrow \widetilde{\mathcal{A}^{\star}}$. The spinor representation of $\mathcal{A} \rightarrow \widetilde{\mathcal{A}^{\star}}$ is $\widetilde{\mathcal{A}^{\star}}=\mathrm{CA}^{\top} \mathrm{C}^{-1}$, where $\mathrm{C}=\mathrm{EW}$. Since $\overline{\widetilde{\mathcal{A}^{\star}}}=\widetilde{(\overline{\mathcal{A}})^{\star}}=\overline{\left(\widetilde{\mathcal{A}^{\star}}\right)}$, then

$$
\overline{\widetilde{\mathrm{A}^{\star}}}=\mathrm{C}\left(\Pi \dot{\mathrm{A}} \Pi^{-1}\right)^{\top} \mathrm{C}^{-1}=\Pi\left(\mathrm{CA}^{\top} \mathrm{C}^{-1}\right) \cdot \Pi^{-1}
$$


or

$$
\overline{\widetilde{\mathrm{A}^{\star}}}=(\mathrm{C} \Pi) \dot{\mathrm{A}}^{\top}(\mathrm{C} \Pi)^{-1}=(\Pi \mathrm{C}) \dot{\mathrm{A}}^{\top}(\Pi \mathrm{C})^{-1},
$$

since $\Pi^{-1}=\Pi^{\top}$ and $\overline{\widetilde{A^{\star}}}=\Pi \dot{C}\left(A^{\top}\right) \dot{C}^{-1} \Pi^{-1}=\Pi C \dot{A}^{\top} C^{-1} \Pi^{-1}$ in the case when $\dot{C}=C$ is a real matrix, and also $\overline{\widetilde{A^{\star}}}=\Pi \dot{C}\left(A^{\top}\right) \cdot \dot{C}^{-1} \Pi^{-1}=$ $\Pi(-C)\left(A^{\top}\right)^{\cdot}\left(-C^{-1}\right) \Pi^{-1}=\Pi C \dot{A}^{\top} C^{-1} \Pi^{-1}$ in the case when $\dot{C}=-C$ is a complex matrix.

Let $\mathrm{F}=\Pi \mathrm{C}$ (or $\mathrm{F}=\Pi \mathrm{EW}$ ) be a matrix of the pseudoantiautomorphism $\mathcal{A} \rightarrow \overline{\widetilde{\mathcal{A}^{\star}}}$. Then the relation (51) can be written as follows

$$
\overline{\widetilde{A}}=F \dot{A}^{\top} F^{-1}
$$

Since under action of the transformation $\mathcal{A} \rightarrow \overline{\widetilde{\mathcal{A}^{\star}}}$ we have $\mathbf{e}_{i} \rightarrow-\mathbf{e}_{i}$, in the spinor representation we must demand $\mathcal{E}_{i} \rightarrow-\mathcal{E}_{i}$ also, or

$$
\varepsilon_{i} \longrightarrow-\varepsilon_{i}=\mathrm{F}_{i}^{\top} \mathrm{F}^{-1}
$$

In case of the real subalgebras $C \ell_{p, q}$ with the $\operatorname{ring} \mathbb{K} \simeq \mathbb{R}, p-q \equiv 0,2(\bmod 8)$, we have $\dot{\mathcal{E}}_{i}=\mathcal{E}_{i}$ for all matrices of the spinbasis and, therefore, the relation (53) takes a form

$$
\varepsilon_{i} \longrightarrow-\varepsilon_{i}=\mathrm{FE}_{i}^{\top} \mathrm{F}^{-1}
$$

Let $\left\{\mathcal{E}_{\gamma_{i}}\right\} \cup\left\{\mathcal{E}_{\delta_{j}}\right\}$ be a spinbasis of the algebra $C \ell_{p, q}$ over the ring $\mathbb{K} \simeq \mathbb{R}$, $\left(\varepsilon_{\gamma_{i}}^{\top}=\mathcal{E}_{\gamma_{i}}, \mathcal{E}_{\delta_{j}}^{\top}=-\mathcal{E}_{\delta_{j}}\right)$. Then the relation (54) can be written in the form

$$
\mathcal{E}_{\gamma_{i}} \longrightarrow-\mathcal{E}_{\gamma_{i}}=\mathrm{F} \mathcal{E}_{\gamma_{i}} \mathrm{~F}^{-1}, \quad \mathcal{E}_{\delta_{j}} \longrightarrow \mathcal{E}_{\delta_{j}}=\mathrm{F}_{\delta_{j}} \mathrm{~F}^{-1}
$$

Whence

$$
\mathcal{E}_{\gamma_{i}} \mathrm{~F}=-\mathrm{F} \mathcal{E}_{\gamma_{i}}, \quad \mathcal{E}_{\delta_{j}} \mathrm{~F}=\mathrm{F} \mathcal{E}_{\delta_{j}}
$$

that is, the matrix $\mathrm{F}$ of the pseudoantiautomorphism $\mathcal{A} \rightarrow \overline{\widetilde{\mathcal{A}^{\star}}}$ in case of the ring $\mathbb{K} \simeq \mathbb{R}$ anticommutes with the symmetric part of the spinbasis of $C \ell_{p, q}$ and commutes with the skewsymmetric part. In virtue of Theorem 3 the matrix $\Pi$ of the pseudoautomorphism $\mathcal{A} \rightarrow \overline{\mathcal{A}}$ over the ring $\mathbb{K} \simeq \mathbb{R}$ is proportional to the unit matrix, $\Pi \simeq \mathrm{I}$. Therefore, in this case $\mathrm{F} \sim \mathrm{C}(\mathrm{F} \sim \mathrm{EW})$ and an explicit form of the matrix $F$ coincides with $C$ (see Theorem 5 ).

In case of the quaternionic ring $\mathbb{K} \simeq \mathbb{H}, p-q \equiv 4,6(\bmod 8)$, the spinbasis of $C \ell_{p, q}$ contains both complex matrices $\mathcal{E}_{\alpha_{t}}$ and real matrices $\mathcal{E}_{\beta_{r}}$. For the complex part the relation (53) takes a form

$$
\mathcal{E}_{\alpha_{t}} \longrightarrow \mathcal{E}_{\alpha_{t}}=\mathrm{F}^{\top} \mathcal{\alpha}_{\alpha_{t}}^{\top} \mathrm{F}^{-1}
$$


Or, taking into account complex symmetric and complex skewsymmetric components of the spinbasis, we obtain

$$
\mathcal{E}_{\alpha_{\gamma}} \longrightarrow \mathcal{E}_{\alpha_{\gamma}}=\mathrm{F}_{\alpha_{\gamma}} \mathrm{F}^{-1}, \quad \mathcal{E}_{\alpha_{\delta}} \longrightarrow \mathcal{E}_{\alpha_{\delta}}=-\mathrm{F} \mathcal{E}_{\alpha_{\delta}} \mathrm{F}^{-1}
$$

Whence

$$
\mathcal{E}_{\alpha_{\gamma}} \mathrm{F}=\mathrm{F} \mathcal{E}_{\alpha_{\gamma}}, \quad \mathcal{E}_{\alpha_{\delta}} \mathrm{F}=-\mathrm{F} \mathcal{E}_{\alpha_{\delta}} .
$$

For the real part of the spinbasis of $C_{p, q}$ from (53) we obtain

$$
\mathcal{E}_{\beta_{r}} \longrightarrow-\mathcal{E}_{\beta_{r}}=\mathrm{F}_{\beta_{r}}^{\top} \mathrm{F}^{-1}
$$

or, taking into account symmetric and skewsymmetric components of the real part of the spinbasis, we find

$$
\mathcal{E}_{\beta_{\gamma}} \longrightarrow-\mathcal{E}_{\beta_{\gamma}}=\mathrm{F} \mathcal{E}_{\beta_{\gamma}} \mathrm{F}^{-1}, \quad \mathcal{E}_{\beta_{\delta}} \longrightarrow \mathcal{E}_{\beta_{\delta}}=\mathrm{F}_{\beta_{\delta}} \mathrm{F}^{-1}
$$

Whence

$$
\mathcal{E}_{\beta_{\gamma}} \mathrm{F}=-\mathrm{F} \mathcal{E}_{\beta_{\gamma}}, \quad \mathcal{E}_{\beta_{\delta}} \mathrm{F}=\mathrm{F} \mathcal{E}_{\beta_{\delta}} .
$$

Therefore, the matrix $\mathrm{F}$ of the pseudoantiautomorphism $\mathcal{A} \rightarrow \overline{\widetilde{\mathcal{A}^{\star}}}$ commutes with the complex symmetric and real skewsymmetric matrices, and also $\mathrm{F}$ anticommutes with the complex skewsymmetric and real symmetric matrices of the spinbasis of $C_{p, q}, p-q \equiv 4,6(\bmod 8)$.

Let us find an explicit form of the matrix $F=\Pi C$. It is easy to see that in virtue of $\mathrm{F}=\Pi C=(\Pi \mathrm{E}) \mathrm{W}=\mathrm{SW}$ the matrix $\mathrm{F}$ is a dual with respect to the matrix $S$ of the pseudoantiautomorphism $\mathcal{A} \rightarrow \overline{\widetilde{\mathcal{A}}}$. In accordance with Theorem 3 for the quaternionic types $p-q \equiv 4,6(\bmod 8)$ the matrix $\Pi$ has two different forms: $\Pi=\mathcal{E}_{\alpha_{1} \alpha_{2} \cdots \alpha_{a}}\left(\dot{\varepsilon}_{\alpha_{t}}=-\mathcal{E}_{\alpha_{t}}\right), a \equiv 0(\bmod 2) ; \Pi=\mathcal{E}_{\beta_{1} \beta_{2} \cdots \beta_{b}}$ $\left(\dot{\mathcal{E}}_{\beta_{r}}=\mathcal{E}_{\beta_{r}}\right), b \equiv 1(\bmod 2)$. In turn, for the quaternionic types the matrix $C$ of the antiautomorphism $\mathcal{A} \rightarrow \widetilde{\mathcal{A}^{\star}}$ has the two forms (see Theorem 5 ): 1) $\mathrm{C}=\mathcal{E}_{i_{1} i_{2} \cdots i_{p+q-k}}$ is the product of all symmetric matrices of the spinbasis of $C \ell_{p, q}$ at $\left.p+q-k \equiv 0(\bmod 2) ; 2\right) \mathrm{C}=\mathcal{E}_{j_{1} j_{2} \cdots j_{k}}$ is the product of all skewsymmetric matrices of the spinbasis at $k \equiv 1(\bmod 2)$. Thus, in accordance with definition $\mathrm{F}=\Pi \mathrm{C}$ we have four products: $\mathrm{F}=\mathcal{E}_{\alpha_{1} \alpha_{2} \cdots \alpha_{a}} \mathcal{E}_{i_{1} i_{2} \cdots i_{p+q-k}}$, $\mathrm{F}=\mathcal{E}_{\alpha_{1} \alpha_{2} \cdots \alpha_{a}} \mathcal{E}_{j_{1} j_{2} \cdots j_{k}}, \mathrm{~F}=\mathcal{E}_{\beta_{1} \beta_{2} \cdots \beta_{b}} \mathcal{E}_{i_{1} i_{2} \cdots i_{p+q-k}}, \mathrm{~F}=\mathcal{E}_{\beta_{1} \beta_{2} \cdots \beta_{b}} \mathcal{E}_{j_{1} j_{2} \cdots j_{k}}$.

Let us examine the first product $\mathrm{F}=\mathcal{E}_{\alpha_{1} \alpha_{2} \cdots \alpha_{a}} \mathcal{E}_{i_{1} i_{2} \cdots i_{p+q-k}}$. In this case $\Pi$ contains all the complex matrices of the spinbasis, among which there are symmetric and skewsymmetric matrices. In turn, C contains all the symmetric matrices, among which there are both complex and real matrices. It is obvious that in this case $\Pi C$ contains a quantity of identical complex symmetric 
matrices. Therefore, the product $\mathrm{F}$ consists of all the complex skewsymmetric matrices and all the real symmetric matrices of the spinbasis. The product $F$ is even, since the original product $\Pi C$ is even also. It is easy to see that $F$ coincides with the product $\mathcal{E}_{d_{1} d_{2} \cdots d_{g}}$ at $g \equiv 0(\bmod 2)$.

Let us find permutation conditions of the matrix $\mathrm{F}=\mathcal{E}_{d_{1} d_{2} \cdots d_{g}}$ with the units of the spinbasis of $C_{p, q}, p-q \equiv 4,6(\bmod 8)$. For the complex and real parts we obtain

$$
\begin{gathered}
\mathrm{F} \mathcal{E}_{\alpha_{\gamma}}=(-1)^{g} \mathcal{E}_{\alpha_{\gamma}} \mathrm{F}, \\
\mathrm{F} \mathcal{E}_{\alpha_{\delta}}=(-1)^{g-\delta} \mathcal{E}_{d_{1} d_{2} \cdots d_{g-2}}, \\
\mathcal{E}_{\alpha_{\delta}} \mathrm{F}=(-1)^{\delta-1} \mathcal{E}_{d_{1} d_{2} \cdots d_{g-2}}, \\
\mathrm{~F} \mathcal{E}_{\beta_{\gamma}}=(-1)^{g-\gamma} \mathcal{E}_{d_{1} d_{2} \cdots d_{g-2}}, \\
\mathcal{E}_{\beta_{\gamma}} \mathrm{F}=(-1)^{\gamma-1} \mathcal{E}_{d_{1} d_{2} \cdots d_{g-2}}, \\
\mathrm{~F} \mathcal{E}_{\beta_{\delta}}=(-1)^{g} \mathcal{E}_{\beta_{\delta}} \mathrm{F} .
\end{gathered}
$$

Therefore, since $g \equiv 0(\bmod 2)$ the matrix $\mathrm{F}$ always commutes with the complex symmetric and real skewsymmetric matrices and always anticommutes with the complex skewsymmetric and real symmetric matrices of the spinbasis. A comparison of the permutation conditions (57)-(60) with the conditions (55)-(56) shows that $\mathrm{F}=\mathcal{E}_{d_{1} d_{2} \cdots d_{g}}$ at $g \equiv 0(\bmod 2)$ automatically satisfies the conditions which define the matrix of the pseudoantiautomorphism $\mathcal{A} \rightarrow \overline{\widetilde{\mathcal{A}^{\star}}}$.

Let examine the second product $\mathrm{F}=\mathcal{E}_{\alpha_{1} \alpha_{2} \cdots \alpha_{a}} \mathcal{E}_{j_{1} j_{2} \cdots j_{k}}$. This product contains all the complex part of the spinbasis and all the skewsymmetric matrices. Therefore, in the product $\Pi C$ there is a quantity of identical complex skewsymmetric matrices. The product $F$ is odd and consists of all the complex symmetric and real skewsymmetric matrices of the spinbasis. It is easy to see that in this case $\mathrm{F}$ coincides with the product $\mathcal{E}_{c_{1} c_{2} \cdots c_{s}}$ at $s \equiv 1(\bmod 2)$. Permutation conditions of the matrix $F=\mathcal{E}_{c_{1} c_{2} \cdots c_{s}}$ with the units of the spinbasis are

$$
\begin{gathered}
\mathrm{F} \mathcal{E}_{\alpha_{\gamma}}=(-1)^{s-\gamma} \mathcal{E}_{c_{1} c_{2} \cdots c_{s-2}}, \\
\mathcal{E}_{\alpha_{\gamma}} \mathrm{F}=(-1)^{\gamma-1} \mathcal{E}_{c_{1} c_{2} \cdots c_{s-2}}, \\
\mathrm{~F} \mathcal{E}_{\alpha_{\delta}}=(-1)^{s} \mathcal{E}_{\alpha_{\delta}} \mathrm{F}, \\
\mathrm{F} \mathcal{E}_{\beta_{\gamma}}=(-1)^{s} \mathcal{E}_{\beta_{\gamma}} \mathrm{F}, \\
\mathrm{F} \mathcal{E}_{\beta_{\delta}}=(-1)^{s-\delta} \mathcal{E}_{c_{1} c_{2} \cdots c_{s-2}}, \\
\mathcal{E}_{\beta_{\delta}} \mathrm{F}=(-1)^{\delta-1} \mathcal{E}_{c_{1} c_{2} \cdots c_{s-2}} .
\end{gathered}
$$

Therefore, since $s \equiv 1(\bmod 2)$ the matrix $\mathrm{F}$ always commutes with the complex symmetric and real skewsymmetric matrices and always anticommutes 
with the complex skewsymmetric and real symmetric matrices of the spinbasis. Comparing the conditions (61) - (64) with the conditions (55) and (56) we see that $\mathrm{F}=\mathcal{E}_{c_{1} c_{2} \cdots c_{s}}$ at $s \equiv 1(\bmod 2)$ identically satisfies the conditions which define the matrix of the transformation $\mathcal{A} \rightarrow \overline{\widetilde{\mathcal{A}^{\star}}}$.

The third product $\mathrm{F}=\Pi \mathrm{C}=\mathcal{E}_{\beta_{1} \beta_{2} \cdots \beta_{b}} \mathcal{E}_{i_{1} i_{2} \cdots i_{p+q-k}}$ contains all the real part and all the symmetric matrices of the spinbasis. Therefore, in the product ПС there is a quantity of identical real symmetric matrices. Thus, the product $\mathrm{F}$ is odd and consists of all the real skewsymmetric and complex symmetric matrices of the spinbasis. It is easy to see that we came again to the matrix $\mathrm{F}=\mathcal{E}_{c_{1} c_{2} \cdots c_{s}}(s \equiv 1(\bmod 2))$ with the permutation conditions (61)-(64).

Finally, the fourth product $F=\mathcal{E}_{\beta_{1} \beta_{2} \cdots \beta_{b}} \mathcal{E}_{j_{1} j_{2} \cdots j_{k}}$ contains all the real part and all the skewsymmetric matrices of the spinbasis. Therefore, in the product $\Pi C$ there is a quantity of identical real skewsymmetric matrices. This product is equivalent to the matrix $\mathrm{F}=\mathcal{E}_{d_{1} d_{2} \cdots d_{g}}(g \equiv 0(\bmod 2))$ with the permutation conditions (57)- (60).

As with the pseudoantiautomorphism $\mathcal{A} \rightarrow \overline{\widetilde{\mathcal{A}}}$, from the four products we have only two non-equivalent products. Let us find squares of the nonequivalent matrices $\mathrm{F}=\mathcal{E}_{d_{1} d_{2} \cdots d_{g}}(g \equiv 0(\bmod 2))$ and $\mathrm{F}=\mathcal{E}_{c_{1} c_{2} \cdots c_{s}}(s \equiv 1$ $(\bmod 2))$ :

$$
\begin{gathered}
\mathrm{F}^{2}=\left(\mathcal{E}_{d_{1} d_{2} \cdots d_{g}}\right)^{2}=\left\{\begin{array}{lll}
+\mathrm{I}, & \text { if } m+v \equiv 0,4 & (\bmod 8), \\
-\mathrm{I}, & \text { if } m+v \equiv 2,6 & (\bmod 8) ;
\end{array}\right. \\
\mathrm{F}^{2}=\left(\mathcal{E}_{c_{1} c_{2} \cdots c_{s}}\right)^{2}=\left\{\begin{array}{lll}
+\mathrm{I}, & \text { if } u+l \equiv 3,7 & (\bmod 8), \\
-\mathrm{I}, & \text { if } u+l \equiv 1,5 & (\bmod 8) .
\end{array}\right.
\end{gathered}
$$

\section{The structure of $\operatorname{Ext}\left(\mathbb{C}_{n}\right)$}

As noted previously, the group $\operatorname{Ext}\left(\mathbb{C}_{n}\right)$ is a finite group of order eight. This group contains as a subgroup the automorphism group $\operatorname{Aut}_{ \pm}\left(\mathbb{C}_{n}\right)$ (reflection group). Moreover, in the case of $\Pi \sim \mathrm{I}$ (when the subalgebra $C \ell_{p, q}$ has the ring $\mathbb{K} \simeq \mathbb{R}, p-q \equiv 0,2(\bmod 8))$ the group $\operatorname{Ext}\left(\mathbb{C}_{n}\right)$ is reduced to its subgroup Aut $_{ \pm}\left(\mathbb{C}_{n}\right)$. The structure of the groups Aut ${ }_{ \pm}\left(\mathbb{C}_{n}\right)$, Aut ${ }_{ \pm}\left(C \ell_{p, q}\right)$ is studied in detail (see Theorems 4 and 5 ).

There are six finite groups of order eight (see the Table 3). One is cyclic and two are direct group products of cyclic groups, hence these three are Abelian. The remaining three groups are the quaternion group $Q_{4}$ with ele-

ments $\{ \pm 1, \pm \mathbf{i}, \pm \mathbf{j}, \pm \mathbf{k}\}$, the dihedral group $D_{4}$, and the group $\stackrel{*}{\mathbb{Z}}_{4} \otimes \mathbb{Z}_{2}$. All these groups are non-Abelian. As known, an important property of each finite 
Table 3. Finite groups of order 8 .

\begin{tabular}{|c|c|c|c|c|}
\hline & Туре & 2 & $\begin{array}{r}\text { rdes } \\
4\end{array}$ & $\begin{array}{l}\text { cture } \\
8\end{array}$ \\
\hline $\begin{array}{l}\text { 1. } \mathbb{Z}_{2} \otimes \mathbb{Z}_{2} \otimes \mathbb{Z}_{2} \\
\text { 2. } \mathbb{Z}_{4} \otimes \mathbb{Z}_{2} \\
\text { 3. } \mathbb{Z}_{8}\end{array}$ & Abelian & 1 & $\begin{array}{l}4 \\
2\end{array}$ & 4 \\
\hline $\begin{array}{l}\text { 4. } D_{4} \\
\text { 5. } Q_{4} \\
\text { 6. } \stackrel{*}{\mathbb{Z}}_{4} \otimes \mathbb{Z}_{2}\end{array}$ & Non-Abelian & 3 & $\begin{array}{l}2 \\
6 \\
4\end{array}$ & \\
\hline
\end{tabular}

group is its order structure. The order of a particular element $\alpha$ in the group is the smallest integer $p$ for which $\alpha^{p}=1$. The Table 3 lists the number of distinct elements in each group which have order 2 , 4 , or 8 (the identity 1 is the only element of order 1$)$. Of course, $\mathbb{Z}_{8}$ does not occur as a $G(p, q)$ (Salingaros group), since every element of $G(p, q)$ has order 1,2 , or 4 . The groups $\mathbb{Z}_{4} \otimes \mathbb{Z}_{2}$ and $\stackrel{*}{\mathbb{Z}}_{4} \otimes \mathbb{Z}_{2}$ have the same order structure, but their signatures $(a, b, c, d, e, f, g)$ are different. Moreover, the group $\stackrel{\mathbb{Z}}{4}_{4} \otimes \mathbb{Z}_{2}$ presents a first example of the finite group of order 8 which has an important physical meaning.

Example 1. Let us consider a Dirac algebra $\mathbb{C}_{4}$. In the algebra $\mathbb{C}_{4}$ we can evolve four different real subalgebras $C_{1,3}, C \ell_{3,1}, C_{4,0}, C \ell_{0,4}$. Let us evolve the spacetime algebra $C \ell_{1,3}$. The algebra $C \ell_{1,3}$ has the quaternionic division $\operatorname{ring} \mathbb{K} \simeq \mathbb{H}(p-q \equiv 6(\bmod 8))$ and, therefore, admits the following spinor 
representation (the well known $\gamma$-basis):

$$
\begin{aligned}
\gamma_{0}=\left(\begin{array}{cccc}
1 & 0 & 0 & 0 \\
0 & 1 & 0 & 0 \\
0 & 0 & -1 & 0 \\
0 & 0 & 0 & -1
\end{array}\right), & \gamma_{1}=\left(\begin{array}{cccc}
0 & 0 & 0 & 1 \\
0 & 0 & 1 & 0 \\
0 & -1 & 0 & 0 \\
-1 & 0 & 0 & 0
\end{array}\right), \\
\gamma_{2}=\left(\begin{array}{cccc}
0 & 0 & 0 & -i \\
0 & 0 & i & 0 \\
0 & i & 0 & 0 \\
-i & 0 & 0 & 0
\end{array}\right), & \gamma_{3}=\left(\begin{array}{cccc}
0 & 0 & 1 & 0 \\
0 & 0 & 0 & -1 \\
-1 & 0 & 0 & 0 \\
0 & 1 & 0 & 0
\end{array}\right)
\end{aligned}
$$

The famous Dirac equation in the $\gamma$-basis looks like

$$
\left(i \gamma_{0} \frac{\partial}{\partial x_{0}}+i \boldsymbol{\gamma} \frac{\partial}{\partial \mathbf{x}}-m\right) \psi\left(x_{0}, \mathbf{x}\right)=0
$$

The invariance of the Dirac equation with respect to $P-, T-$, and $C-$ transformations leads to the following representation (see, for example, 9] and also many other textbooks on quantum field theory):

$$
P \sim \gamma_{0}, \quad T \sim \gamma_{1} \gamma_{3}, \quad C \sim \gamma_{2} \gamma_{0}
$$

Thus, we can form a finite group of order 8 (CPT-group)

$$
\begin{aligned}
\{1, P, T, P T, C, C P, C T, C P T\} & \sim \\
& \sim\left\{1, \gamma_{0}, \gamma_{1} \gamma_{3}, \gamma_{0} \gamma_{1} \gamma_{3}, \gamma_{2} \gamma_{0}, \gamma_{2}, \gamma_{2} \gamma_{0} \gamma_{1} \gamma_{3}, \gamma_{2} \gamma_{1} \gamma_{3}\right\}
\end{aligned}
$$

It is easy to verify that a multiplication table of this group has a form

\begin{tabular}{|c||c|c|c|c|c|c|c|c|}
\hline & 1 & $\gamma_{0}$ & $\gamma_{13}$ & $\gamma_{013}$ & $\gamma_{20}$ & $\gamma_{2}$ & $\gamma_{2013}$ & $\gamma_{213}$ \\
\hline \hline 1 & 1 & $\gamma_{0}$ & $\gamma_{13}$ & $\gamma_{013}$ & $\gamma_{2}$ & $\gamma_{2}$ & $\gamma_{2013}$ & $\gamma_{213}$ \\
\hline$\gamma_{0}$ & $\gamma_{0}$ & 1 & $\gamma_{013}$ & $\gamma_{13}$ & $-\gamma_{2}$ & $-\gamma_{20}$ & $-\gamma_{213}$ & $-\gamma_{2013}$ \\
\hline$\gamma_{13}$ & $\gamma_{13}$ & $\gamma_{013}$ & -1 & $-\gamma_{0}$ & $\gamma_{2013}$ & $\gamma_{213}$ & $-\gamma_{20}$ & $-\gamma_{2}$ \\
\hline$\gamma_{013}$ & $\gamma_{013}$ & $\gamma_{13}$ & $-\gamma_{0}$ & -1 & $-\gamma_{213}$ & $-\gamma_{2013}$ & $\gamma_{2}$ & $\gamma_{20}$ \\
\hline$\gamma_{20}$ & $\gamma_{20}$ & $\gamma_{2}$ & $\gamma_{2013}$ & $\gamma_{213}$ & 1 & $\gamma_{0}$ & $\gamma_{13}$ & $\gamma_{013}$ \\
\hline$\gamma_{2}$ & $\gamma_{2}$ & $\gamma_{20}$ & $\gamma_{213}$ & $\gamma_{2013}$ & $-\gamma_{0}$ & -1 & $-\gamma_{013}$ & $-\gamma_{13}$ \\
\hline$\gamma_{2013}$ & $\gamma_{2013}$ & $\gamma_{213}$ & $-\gamma_{20}$ & $\gamma_{2}$ & $\gamma_{13}$ & $\gamma_{013}$ & -1 & $-\gamma_{0}$ \\
\hline$\gamma_{213}$ & $\gamma_{213}$ & $\gamma_{2013}$ & $-\gamma_{2}$ & $-\gamma_{20}$ & $-\gamma_{013}$ & $-\gamma_{13}$ & $\gamma_{0}$ & 1 \\
\hline
\end{tabular}




\begin{tabular}{|c||c|c|c|c|c|c|c|c|}
\hline & 1 & $P$ & $T$ & $P T$ & $C$ & $C P$ & $C T$ & $C P T$ \\
\hline \hline 1 & 1 & $P$ & $T$ & $P T$ & $C$ & $C P$ & $C T$ & $C P T$ \\
\hline$P$ & $P$ & 1 & $P T$ & $T$ & $-C P$ & $-C$ & $-C P T$ & $-C T$ \\
\hline$T$ & $T$ & $P T$ & -1 & $-P$ & $C T$ & $C P T$ & $-C$ & $-C P$ \\
\hline$P T$ & $P T$ & $T$ & $-P$ & -1 & $-C P T$ & $-C T$ & $C P$ & $C$ \\
\hline$C$ & $C$ & $C P$ & $C T$ & $C P T$ & 1 & $P$ & $T$ & $P T$ \\
\hline$C P$ & $C P$ & $C$ & $C P T$ & $C T$ & $-P$ & -1 & $-P T$ & $-T$ \\
\hline$C T$ & $C T$ & $C P T$ & $-C$ & $C P$ & $T$ & $P T$ & -1 & $-P$ \\
\hline$C P T$ & $C P T$ & $C T$ & $-C P$ & $-C$ & $-P T$ & $-T$ & $P$ & 1 \\
\hline
\end{tabular}

Hence it follows that the CPT-group (69) is a non-Abelian finite group of the order structure $(3,4)$. In more details, it is the group $\mathbb{Z}_{4}^{*} \otimes \mathbb{Z}_{2}$ with the signature $(+,-,-,+,-,-,+)$.

Theorem 6. Let $\mathbb{C}_{n}$ be the Clifford algebra over the field $\mathbb{F}=\mathbb{C}$ and let $\operatorname{Ext}\left(\mathbb{C}_{n}\right)=\{\mathrm{I}, \mathrm{W}, \mathrm{E}, \mathrm{C}, \Pi, \mathrm{K}, \mathrm{S}, \mathrm{F}\}$ be an extended automorphism group of the algebra $\mathbb{C}_{n}$, where $\mathrm{W}, \mathrm{E}, \mathrm{C}, \Pi, \mathrm{K}, \mathrm{S}, \mathrm{F}$ are spinor representations of the transformations $\mathcal{A} \rightarrow \mathcal{A}^{\star}, \mathcal{A} \rightarrow \widetilde{\mathcal{A}}, \mathcal{A} \rightarrow \widetilde{\mathcal{A}^{\star}}, \mathcal{A} \rightarrow \overline{\mathcal{A}}, \mathcal{A} \rightarrow \overline{\mathcal{A}^{\star}}, \mathcal{A} \rightarrow \overline{\widetilde{\mathcal{A}}}, \mathcal{A} \rightarrow \overline{\widetilde{\mathcal{A}^{\star}}}$. Then over the field $\mathbb{F}=\mathbb{C}$ in dependence on a division ring structure of the real subalgebras $C_{p, q} \subset \mathbb{C}_{n}$ there exist following isomorphisms between finite groups and groups $\operatorname{Ext}\left(\mathbb{C}_{n}\right)$ :

1) $\mathbb{K} \simeq \mathbb{R}$, types $p-q \equiv 0,2(\bmod 8)$.

In this case the matrix $\Pi$ of the pseudoautomorphism $\mathcal{A} \rightarrow \overline{\mathcal{A}}$ is proportional to the unit matrix (identical transformation) and the extended automorphism group $\operatorname{Ext}\left(\mathbb{C}_{n}\right)$ is reduced to the group of fundamental automorphisms, $\operatorname{Aut}_{ \pm}\left(\mathbb{C}_{n}\right)$.

2) $\mathbb{K} \simeq \mathbb{H}$, types $p-q \equiv 4,6(\bmod 8)$.

In dependence on a spinbasis structure of the subalgebra $C_{p, q}$ there exist the following group isomorphisms: Ext $-\left(\mathbb{C}_{n}\right) \simeq \mathbb{Z}_{2} \otimes \mathbb{Z}_{2} \otimes \mathbb{Z}_{2}$ with the signature $(+,+,+,+,+,+,+)$ and $\operatorname{Ext}\left(\mathbb{C}_{n}\right) \simeq \mathbb{Z}_{4} \otimes \mathbb{Z}_{2}$ with $(+,+,+,-,-,-,-)$ for the type $p-q \equiv 4(\bmod 8)$ if $m, v, l, u \equiv 0(\bmod 2)$, where $m$ and $l$ are quantities of complex skewsymmetric and symmetric matrices, and $u$ and $v$ are quantities of real skewsymmetric and symmetric matrices of the spinbasis of $C_{p, q}$. Correspondingly, at $m, v, l, u \equiv 0(\bmod 2)$ there exist Abelian groups Ext $_{-}\left(\mathbb{C}_{n}\right) \simeq \mathbb{Z}_{4} \otimes \mathbb{Z}_{2}$ with $(+,-,-, d, e, f, g)$ for the type $p-q \equiv 4(\bmod 8)$ and 
$\operatorname{Ext}_{-}\left(\mathbb{C}_{n}\right) \simeq \mathbb{Z}_{4} \otimes \mathbb{Z}_{2}$ with $(-,+,-, d, e, f, g),(-,-,+, d, e, f, g)$ for the type $p-q \equiv 6(\bmod 8)$, where among the symbols $d, e, f, g$ there are two pluses and two minuses.

If $m, v, l, u \equiv 1(\bmod 2)$ or if among $m, v, l, u$ there are both even and odd numbers, then there exists the non-Abelian group $\operatorname{Ext}_{+}\left(\mathbb{C}_{n}\right) \simeq Q_{4}$ with the signatures $(-,-,-, d, e, f, g)$ for the type $p-q \equiv 6(\bmod 8)$, where among $d, e, f, g$ there are one plus and three minuses. And also there exist $\operatorname{Ext}_{+}\left(\mathbb{C}_{n}\right) \simeq D_{4}$ with $(+,-,+, d, e, f, g),(+,+,-, d, e, f, g)$ for the type $p-q \equiv 4(\bmod 8)$ and $\operatorname{Ext}_{+}\left(\mathbb{C}_{n}\right) \simeq D_{4}$ with $(-,+,+, d, e, f, g)$ for the type $p-q \equiv 6(\bmod 8)$, where among the symbols $d, e, f, g$ there are three pluses and one minus. Besides, there exist the groups $\operatorname{Ext}_{+}\left(\mathbb{C}_{n}\right) \simeq Q_{4}$ with the signatures $(+,-,-,-,-,-,-)$ for $p-q \equiv 4(\bmod 8)$ and $(-,+,-,-,-,-,-)$, $(-,-,+,-,-,-,-)$ for $p-q \equiv 6(\bmod 8)$. And also there exist the groups $\operatorname{Ext}_{+}\left(\mathbb{C}_{n}\right) \simeq D_{4}$ with $(+,+,+, d, e, f, g),(+,-,-,+,+,+,+)$ for the type $p-q \equiv 4(\bmod 8)$ and $(a, b, c,+,+,+,+)$ for the type $p-q \equiv 6(\bmod 8)$ (among the symbols $a, b, c$ there are one plus and two minuses, and among $d, e, f, g$ there are two pluses and two minuses). There exists the non-Abelian group $\operatorname{Ext}_{+}\left(\mathbb{C}_{n}\right) \simeq \stackrel{*}{\mathbb{Z}}_{4} \otimes \mathbb{Z}_{2}$ with the signatures $(+,-,-, d, e, f, g)$ for the type $p-q \equiv 4(\bmod 8)$ and $(-,+,-, d, e, f, g),(-,-,+, d, e, f, g)$ for the type $p-q \equiv 6(\bmod 8)$, where among $d, e, f, g$ there are two pluses and two minuses. And also there exist $\operatorname{Ext}_{+}\left(\mathbb{C}_{n}\right) \simeq \mathbb{Z}_{4}^{*} \otimes \mathbb{Z}_{2}$ with $(+,-,+, d, e, f, g)$, $(+,+,-, d, e, f, g)$ for $p-q \equiv 4(\bmod 8)$ and $(-,+,+, d, e, f, g)$ for $p-q \equiv 6$ $(\bmod 8)$, where among $d, e, f, g$ there are one plus and three minuses. Finally, for the type $p-q \equiv 6(\bmod 8)$ there exists $\operatorname{Ext}_{+}\left(\mathbb{C}_{n}\right) \simeq \stackrel{*}{\mathbb{Z}}_{4} \otimes \mathbb{Z}_{2}$ with $(-,-,-, d, e, f, g)$, where among the symbols $d, e, f, g$ there are three pluses and one minus. The full number of the different signatures $(a, b, c, d, e, f, g)$ is equal to 64 .

Proof. First of all, it is necessary to define permutation relations between the elements of the group Ext. We start with the matrix of the pseudoautomorphism $\mathcal{A} \rightarrow \overline{\mathcal{A}}$ (permutation relations between the elements $\mathrm{W}, \mathrm{E}$ and $\mathrm{C}$ are found in Theorem 5). As known, for the types $p-q \equiv 4,6(\bmod 8)$ the matrix $\Pi$ exists in the two different forms: 1) $\Pi=\mathcal{E}_{\alpha_{1} \alpha_{2} \cdots \alpha_{a}}$ is the product of all complex matrices of the spinbasis at $a \equiv 0(\bmod 2) ; 2) \Pi=\mathcal{E}_{\beta_{1} \beta_{2} \cdots \beta_{b}}$ is the product of all real matrices of the spinbasis at $b \equiv 1(\bmod 2)$.

Let us consider permutation relations of $\Pi$ with the matrix $\mathrm{K}$ of the pseudoautomorphism $\mathcal{A} \rightarrow \overline{\mathcal{A}^{\star}}$. The matrix $\mathrm{K}$ also exists in the two different forms: $\mathrm{K}=\mathcal{E}_{\alpha_{1} \alpha_{2} \cdots \alpha_{a}}$ at $a \equiv 1(\bmod 2)$ and $\mathrm{K}=\mathcal{E}_{\beta_{1} \beta_{2} \cdots \beta_{b}}$ at $b \equiv 0(\bmod 2)$. In virtue of the definition $\mathrm{K}=\Pi \mathrm{W}$, where $\mathrm{W}=\mathcal{E}_{12 \cdots p+q}$ is the spinor representation of 
the automorphism $\mathcal{A} \rightarrow \mathcal{A}^{\star}$, the matrix $\Pi=\mathcal{E}_{\alpha_{1} \alpha_{2} \cdots \alpha_{a}}$ corresponds to a matrix $\mathrm{K}=\mathcal{E}_{\beta_{1} \beta_{2} \cdots \beta_{b}}$, since $n=p+q$ is always even for the types $p-q \equiv 4,6(\bmod 8)$. Correspondingly, for the matrix $\Pi=\mathcal{E}_{\beta_{1} \beta_{2} \cdots \beta_{b}}$ we obtain $\mathrm{K}=\mathcal{E}_{\alpha_{1} \alpha_{2} \cdots \alpha_{a}}$, where $a, b \equiv 1(\bmod 2)$. It is easy to see that in both cases we have a relation

$$
\Pi \mathrm{K}=(-1)^{a b} \mathrm{~K} \Pi
$$

that is, at $a, b \equiv 0(\bmod 2)$ the matrices $\Pi$ and $\mathrm{K}$ always commute and at $a, b \equiv 1(\bmod 2)$ always anticommute.

Let us find now permutation relations of $\Pi$ with the matrix $S$ of the pseudoantiautomorphism $\mathcal{A} \rightarrow \overline{\widetilde{\mathcal{A}}}$. As known, the matrix $S$ exists in the two nonequivalent forms: 1) $S=\mathcal{E}_{c_{1} c_{2} \cdots c_{s}}$ is the product of all complex symmetric and real skewsymmetric matrices at $s \equiv 0(\bmod 2) ; 2) \mathrm{S}=\mathcal{E}_{d_{1} d_{2} \cdots d_{g}}$ is the product of all complex skewsymmetric and real symmetric matrices at $g \equiv 1(\bmod 2)$. From $S=\Pi E$ it follows that $\Pi=\mathcal{E}_{\alpha_{1} \alpha_{2} \cdots \alpha_{a}}$ corresponds to $S=\mathcal{E}_{c_{1} c_{2} \cdots c_{s}}$ if $\mathrm{E}=\mathcal{E}_{j_{1} j_{2} \cdots j_{k}}$ and to $\mathrm{S}=\mathcal{E}_{d_{1} d_{2} \cdots d_{g}}$ if $\mathrm{E}=\mathcal{E}_{i_{1} i_{2} \cdots i_{p+q-k}}$. In turn, the matrix $\Pi=\mathcal{E}_{\beta_{1} \beta_{2} \cdots \beta_{b}}$ corresponds to $\mathrm{S}=\mathcal{E}_{d_{1} d_{2} \cdots d_{g}}$ if $\mathrm{E}=\mathcal{E}_{j_{1} j_{2} \cdots j_{k}}$ and to $\mathrm{S}=\mathcal{E}_{c_{1} c_{2} \cdots c_{S}}$ if $\mathrm{E}=\mathcal{E}_{i_{1} i_{2} \cdots i_{p+q-k}}$. Thus, taking into account that $\mathrm{S}=\Pi \mathrm{E}$, we obtain

$$
\begin{aligned}
& \Pi \mathrm{S}=(-1)^{\frac{a(a-1)}{2}+\tau} \mathcal{E}_{j_{1} j_{2} \cdots j_{k}}, \\
& \mathrm{S \Pi}=(-1)^{\frac{a(a-1)}{2}+\tau-m+a k} \mathcal{E}_{j_{1} j_{2} \cdots j_{k}}
\end{aligned}
$$

for the matrices $\Pi=\mathcal{E}_{\alpha_{1} \alpha_{2} \cdots \alpha_{a}}$ and $S=\mathcal{E}_{c_{1} c_{2} \cdots c_{s}}$, where $m$ is the number of complex skewsymmetric matrices of the spinbasis of $C \ell_{p, q}, p-q \equiv 4,6$ $(\bmod 8)$. Since a comparison $a k \equiv 0(\bmod 2)$ holds always, then the matrices $\Pi$ and $\mathrm{S}$ commute at $m \equiv 0(\bmod 2)$ and anticommute at $m \equiv 1(\bmod 2)$. Correspondingly,

$$
\begin{aligned}
& \Pi \mathrm{\Pi}=(-1)^{\frac{a(a-1)}{2}+\tau} \mathcal{E}_{i_{1} i_{2} \cdots i_{p+q-k}}, \\
& \mathrm{S \Pi}=(-1)^{\frac{a(a-1)}{2}+\tau-l+a(p+q-k)} \mathcal{E}_{i_{1} i_{2} \cdots i_{p+q-k}}
\end{aligned}
$$

for the matrices $\Pi=\mathcal{E}_{\alpha_{1} \alpha_{2} \cdots \alpha_{a}}$ and $S=\mathcal{E}_{d_{1} d_{2} \cdots d_{g}}$, where $l$ is the number of complex symmetric matrices of the spinbasis. Since $a(p+q-k) \equiv 0(\bmod 2)$ $(a \equiv 0(\bmod 2), p+q-k \equiv 1(\bmod 2))$, then in this case the matrices $\Pi$ and $\mathrm{S}$ commute at $l \equiv 0(\bmod 2)$ and anticommute at $l \equiv 1(\bmod 2)$. Further, we have

$$
\begin{aligned}
& \Pi \mathrm{S}=(-1)^{\frac{b(b-1)}{2}+\tau} \mathcal{E}_{j_{1} j_{2} \cdots j_{k}}, \\
& \mathrm{S \Pi}=(-1)^{\frac{b(b-1)}{2}+\tau-u+b k} \mathcal{E}_{j_{1} j_{2} \cdots j_{k}}
\end{aligned}
$$


for the matrices $\Pi=\mathcal{E}_{\beta_{1} \beta_{2} \cdots \beta_{b}}$ and $\mathbf{S}=\mathcal{E}_{d_{1} d_{2} \cdots d_{g}}$, where $u$ is the number of real skewsymmetric matrices of the spinbasis. Since $b k \equiv 0(\bmod 2)(b \equiv 1$ $(\bmod 2), k \equiv 0(\bmod 2))$, then $\Pi$ and $\mathbf{S}$ commute at $u \equiv 0(\bmod 2)$ and anticommute at $u \equiv 1(\bmod 2)$. Finally,

$$
\begin{aligned}
& \text { ПS }=(-1)^{\frac{b(b-1)}{2}+\tau} \mathcal{E}_{i_{1} i_{2} \cdots i_{p+q-k}}, \\
& \text { SП }=(-1)^{\frac{b(b-1)}{2}+\tau-v+b(p+q-k)} \mathcal{E}_{i_{1} i_{2} \cdots i_{p+q-k}}
\end{aligned}
$$

for the matrices $\Pi=\mathcal{E}_{\beta_{1} \beta_{2} \cdots \beta_{b}}$ and $S=\mathcal{E}_{c_{1} c_{2} \cdots c_{s}}$, where $v$ is the number of real symmetric matrices. Therefore, permutation conditions of the matrices $\Pi$ and $\mathrm{S}$ in this case have the form $b(p+q-k) \equiv v(\bmod 2)$, that is, $\Pi$ and $\mathbf{S}$ commute at $v \equiv 1(\bmod 2)$ and anticommute at $v \equiv 0(\bmod 2)$, since $b, p+q-k \equiv 1$ $(\bmod 2)$.

Now we find permutation conditions of $\Pi$ with the matrix $F$ of the pseudoantiautomorphism $\mathcal{A} \rightarrow \overline{\widetilde{\mathcal{A}^{\star}}}$. In turn, the matrix $\mathrm{F}$ exists also in the two non-equivalent forms: $\mathrm{F}=\mathcal{E}_{d_{1} d_{2} \cdots d_{g}}$ at $g \equiv 0(\bmod 2)$ and $\mathrm{F}=\mathcal{E}_{c_{1} c_{2} \cdots c_{s}}$ at $s \equiv 1(\bmod 2)$. From the definition $\mathrm{F}=\Pi \mathrm{C}$ it follows that $\Pi=\mathcal{E}_{\alpha_{1} \alpha_{2} \cdots \alpha_{a}}$ corresponds to $\mathrm{F}=\mathcal{E}_{d_{1} d_{2} \cdots d_{g}}$ if $\mathrm{C}=\mathcal{E}_{i_{1} i_{2} \cdots i_{p+q-k}}$ and to $\mathrm{F}=\mathcal{E}_{c_{1} c_{2} \cdots c_{s}}$ if $\mathrm{C}=\mathcal{E}_{j_{1} j_{2} \cdots j_{k}}$. The matrix $\Pi=\mathcal{E}_{\beta_{1} \beta_{2} \cdots \beta_{b}}$ corresponds to $\mathrm{F}=\mathcal{E}_{c_{1} c_{2} \cdots c_{s}}$ if $\mathrm{C}=\mathcal{E}_{i_{1} i_{2} \cdots i_{p+q-k}}$ and to $\mathrm{F}=\mathcal{E}_{d_{1} d_{2} \cdots d_{g}}$ if $\mathrm{C}=\mathcal{E}_{j_{1} j_{2} \cdots j_{k}}$. Thus, taking into account that $\mathrm{F}=\Pi \mathrm{C}$, we obtain

$$
\begin{aligned}
& \Pi \mathrm{F}=(-1)^{\frac{a(a-1)}{2}+\tau} \mathcal{E}_{i_{1} i_{2} \cdots i_{p+q-k}}, \\
& \mathrm{~F} \Pi=(-1)^{\frac{a(a-1)}{2}+\tau-l+a(p+q-k)} \mathcal{E}_{i_{1} i_{2} \cdots i_{p+q-k}}
\end{aligned}
$$

for the matrices $\Pi=\mathcal{E}_{\alpha_{1} \alpha_{2} \cdots \alpha_{a}}$ and $F=\mathcal{E}_{d_{1} d_{2} \cdots d_{g}}$. It is easy to see that $\Pi$ and $\mathrm{F}$ commute at $l \equiv 0(\bmod 2)$ and anticommute at $l \equiv 1(\bmod 2)$, since $a, p+q-k \equiv 0(\bmod 2)$. Analogously,

$$
\begin{aligned}
& \Pi \mathrm{F}=(-1)^{\frac{a(a-1)}{2}+\tau} \mathcal{E}_{j_{1} j_{2} \cdots j_{k}}, \\
& \mathrm{~F} \Pi=(-1)^{\frac{a(a-1)}{2}+\tau-m+a k} \mathcal{E}_{j_{1} j_{2} \cdots j_{k}}
\end{aligned}
$$

for $\Pi=\mathcal{E}_{\alpha_{1} \alpha_{2} \cdots \alpha_{a}}$ and $\mathbf{F}=\mathcal{E}_{c_{1} c_{2} \cdots c_{s}}$. Therefore, $\Pi$ and $\mathbf{F}$ commute at $m \equiv 0$ $(\bmod 2)$ and anticommute at $m \equiv 1(\bmod 2)$, since $a \equiv 0(\bmod 2), k \equiv 1$ $(\bmod 2)$. Further, we have

$$
\begin{aligned}
& \Pi \mathrm{F}=(-1)^{\frac{b(b-1)}{2}+\tau} \mathcal{E}_{i_{1} i_{2} \cdots i_{p+q-k}}, \\
& \mathrm{~F} \Pi=(-1)^{\frac{b(b-1)}{2}+\tau-v+b(p+q-k)} \mathcal{E}_{i_{1} i_{2} \cdots i_{p+q-k}}
\end{aligned}
$$


for the matrices $\Pi=\mathcal{E}_{\beta_{1} \beta_{1} \cdots \beta_{b}}$ and $F=\mathcal{E}_{c_{1} c_{2} \cdots c_{s}}$. In this case, $\Pi$ and $F$ commute at $v \equiv 0(\bmod 2)$ and anticommute at $v \equiv 1(\bmod 2)$, since $b \equiv 1(\bmod 2)$, $p+q-k \equiv 0(\bmod 2)$. Finally,

$$
\begin{aligned}
& \Pi \mathrm{F}=(-1)^{\frac{b(b-1)}{2}+\tau} \mathcal{E}_{j_{1} j_{2} \cdots j_{k}}, \\
& \mathrm{~F} \Pi=(-1)^{\frac{b(b-1)}{2}+\tau-u+b k} \mathcal{E}_{j_{1} j_{2} \cdots j_{k}}
\end{aligned}
$$

for $\Pi=\mathcal{E}_{\beta_{1} \beta_{2} \cdots \beta_{b}}$ and $\mathrm{F}=\mathcal{E}_{d_{1} d_{2} \cdots d_{g}}$. Therefore, in this case permutation conditions of the matrices $\Pi$ and $\mathrm{F}$ have the form $b k \equiv u(\bmod 2)$, that is, $\Pi$ and $\mathrm{F}$ commute at $u \equiv 1(\bmod 2)$ and anticommute at $u \equiv 0(\bmod 2)$, since $b, k \equiv 1$ $(\bmod 2)$.

Let us define now permutation conditions of $\Pi$ with the matrices $\mathrm{W}, \mathrm{E}$ and $\mathrm{C}$ of the transformations $\mathcal{A} \rightarrow \mathcal{A}^{\star}, \mathcal{A} \rightarrow \widetilde{\mathcal{A}}$ and $\mathcal{A} \rightarrow \widetilde{\mathcal{A}^{\star}}$, respectively. First of all, according to Theorem 3 in the case of subalgebras $C \ell_{p, q}$ with the real $\operatorname{ring} \mathbb{K} \simeq \mathbb{R}($ types $p-q \equiv 0,2(\bmod 8))$ the matrix $\Pi$ is proportional to the unit matrix and, therefore, $\Pi$ commutes with $\mathrm{W}, \mathrm{E}$ and $\mathrm{C}$. In case of the ring $\mathbb{K} \simeq \mathbb{H}($ types $p-q \equiv 4,6(\bmod 8))$ the matrix $\Pi$ exists in the two forms: $\Pi_{a}$ at $a \equiv 0(\bmod 2)$ and $\Pi_{b}$ at $b \equiv 1(\bmod 2)$. Since $a+b=p+q$, then the matrix $W$ can be represented by a product $\mathcal{E}_{\alpha_{1} \alpha_{2} \cdots \alpha_{a}} \mathcal{E}_{\beta_{1} \beta_{2} \cdots \beta_{b}}$ and for $\Pi=\mathcal{E}_{\alpha_{1} \alpha_{2} \cdots \alpha_{a}}$ we have

$$
\begin{aligned}
& \Pi \mathrm{W}=(-1)^{\frac{a(a-1)}{2}} \sigma\left(\alpha_{1}\right) \sigma\left(\alpha_{2}\right) \cdots \sigma\left(\alpha_{a}\right) \mathcal{E}_{\beta_{1} \beta_{2} \cdots \beta_{b}}, \\
& \mathrm{~W} \Pi=(-1)^{\frac{a(a-1)}{2}+b a} \sigma\left(\alpha_{1}\right) \sigma\left(\alpha_{2}\right) \cdots \sigma\left(\alpha_{a}\right) \mathcal{E}_{\beta_{1} \beta_{2} \cdots \alpha_{b}} .
\end{aligned}
$$

Hence it follows that $\Pi$ and $\mathrm{W}$ commute at $a b \equiv 0(\bmod 2)$ and anticommute at $a b \equiv 1(\bmod 2)$, but $a \equiv 0(\bmod 2)$ and, therefore, the matrices $\Pi$ and W always commute in this case. Taking $\Pi=\mathcal{E}_{\beta_{1} \beta_{2} \cdots \beta_{b}}$, we find the following conditions

$$
\begin{aligned}
& \Pi \mathrm{W}=(-1)^{\frac{b(b-1)}{2}+a b} \sigma\left(\beta_{1}\right) \sigma\left(\beta_{2}\right) \cdots \sigma\left(\beta_{b}\right) \mathcal{E}_{\alpha_{1} \alpha_{2} \cdots \alpha_{a}}, \\
& \mathrm{~W} \Pi=(-1)^{\frac{b(b-1)}{2}} \sigma\left(\beta_{1}\right) \sigma\left(\beta_{2}\right) \cdots \sigma\left(\beta_{b}\right) \mathcal{E}_{\alpha_{1} \alpha_{2} \cdots \alpha_{a}} .
\end{aligned}
$$

Hence it follows that $a b \equiv 1(\bmod 2)$, since in this case $a, b \equiv 1(\bmod 2)$ $(p+q=a+b$ is an even number). Therefore, at $b \equiv 1(\bmod 2)$ the matrices $\Pi$ and $W$ always anticommute.

Let us find now permutation conditions between $\Pi$ and the matrix $\mathrm{E}$ of the antiautomorphism $\mathcal{A} \rightarrow \widetilde{\mathcal{A}}$. As known (see Theorem $[$ ), the matrix E exists in the two non-equivalent forms. First of all, if $\Pi=\mathcal{E}_{\alpha_{1} \alpha_{2} \cdots \alpha_{a}}$ and $E=\mathcal{E}_{j_{1} j_{2} \cdots j_{k}}$, then it is obvious that $\Pi$ and $\mathbf{E}$ contain $m$ identical complex skewsymmetric matrices. We can represent the matrices $\Pi$ and $E$ in the form of the following 
products: $\Pi=\mathcal{E}_{\alpha_{1} \alpha_{2} \cdots \alpha_{l}} \mathcal{E}_{i_{1} i_{2} \cdots i_{m}}$ and $\mathrm{E}=\mathcal{E}_{i_{1} i_{2} \cdots i_{m}} \mathcal{E}_{j_{1} j_{2} \cdots j_{u}}$, where $l$ and $u$ are the numbers of complex symmetric and real skewsymmetric matrices, respectively. Therefore,

$$
\begin{aligned}
& \text { ПЕ }=(-1)^{\frac{m(m-1)}{2}} \sigma\left(i_{1}\right) \sigma\left(i_{2}\right) \cdots \sigma\left(i_{m}\right) \mathcal{E}_{\alpha_{1} \alpha_{2} \cdots \alpha_{l}} \mathcal{E}_{j_{1} j_{2} \cdots j_{u}} \\
& \mathrm{E \Pi}=(-1)^{\frac{m(m-1)}{2}+m(u+l)} \sigma\left(i_{1}\right) \sigma\left(i_{2}\right) \cdots \sigma\left(i_{m}\right) \mathcal{E}_{\alpha_{1} \alpha_{2} \cdots \alpha_{l}} \mathcal{E}_{j_{1} j_{2} \cdots j_{u}}
\end{aligned}
$$

that is, $\Pi$ and $\mathrm{E}$ commute at $m(u+l) \equiv 0(\bmod 2)$ and anticommute at $m(u+l) \equiv 1(\bmod 2)$.

Analogously, if $\Pi=\mathcal{E}_{\alpha_{1} \alpha_{2} \cdots \alpha_{a}}$ and $E=\mathcal{E}_{i_{1} i_{2} \cdots i_{p+q} k}$, then it is easy to see that in this case $\Pi$ and $E$ contain $l$ identical complex symmetric matrices. Then $\Pi=\mathcal{E}_{\alpha_{1} \alpha_{2} \cdots \alpha_{m}} \mathcal{E}_{i_{1} i_{2} \cdots i_{l}}, \mathrm{E}=\mathcal{E}_{i_{1} i_{2} \cdots i_{l}} \mathcal{E}_{i_{1} i_{2} \cdots i_{v}}$ ( $v$ is the number of all real symmetric matrices of the spinbasis) and

$$
\begin{aligned}
& \text { ПЕ }=(-1)^{\frac{l(l-1)}{2}} \sigma\left(i_{1}\right) \sigma\left(i_{2}\right) \cdots \sigma\left(i_{l}\right) \mathcal{E}_{\alpha_{1} \alpha_{2} \cdots \alpha_{m}} \mathcal{E}_{i_{1} i_{2} \cdots i_{v}}, \\
& \mathrm{E \Pi}=(-1)^{\frac{l(l-1)}{2}+l(m+v)} \sigma\left(i_{1}\right) \sigma\left(i_{2}\right) \cdots \sigma\left(i_{l}\right) \mathcal{E}_{\alpha_{1} \alpha_{2} \cdots \alpha_{m}} \mathcal{E}_{i_{1} i_{2} \cdots i_{v}} .
\end{aligned}
$$

Therefore, in this case $\Pi$ and $\mathrm{E}$ commute at $l(m+v) \equiv 0(\bmod 2)$ and anticommute at $l(m+v) \equiv 1(\bmod 2)$.

In turn, the matrices $\Pi=\mathcal{E}_{\beta_{1} \beta_{2} \cdots \beta_{b}}$ and $\mathrm{E}=\mathcal{E}_{j_{1} j_{2} \cdots j_{k}}$ contain $u$ identical real skewsymmetric matrices. Therefore, $\Pi=\mathcal{E}_{\beta_{1} \beta_{2} \cdots \beta_{v}} \mathcal{E}_{i_{1} i_{2} \cdots i_{u}}, \mathrm{E}=\mathcal{E}_{i_{1} i_{2} \cdots i_{u}} \mathcal{E}_{j_{1} j_{2} \cdots j_{m}}$ and

$$
\begin{aligned}
& \text { ПЕ }=(-1)^{\frac{u(u-1)}{2}} \sigma\left(i_{1}\right) \sigma\left(i_{2}\right) \cdots \sigma\left(i_{u}\right) \mathcal{E}_{\beta_{1} \beta_{2} \cdots \beta_{v}} \mathcal{E}_{j_{1} j_{2} \cdots j_{m}}, \\
& \mathrm{E \Pi}=(-1)^{\frac{u(u-1)}{2}+u(m+v)} \sigma\left(i_{1}\right) \sigma\left(i_{2}\right) \cdots \sigma\left(i_{u}\right) \mathcal{E}_{\beta_{1} \beta_{2} \cdots \beta_{v}} \mathcal{E}_{j_{1} j_{2} \cdots j_{m}} .
\end{aligned}
$$

Hence it follows that $\Pi$ and $\mathrm{E}$ commute at $u(m+v) \equiv 0(\bmod 2)$ and anticommute at $u(m+v) \equiv 1(\bmod 2)$.

Finally, the matrices $\Pi=\mathcal{E}_{\beta_{1} \beta_{2} \cdots \beta_{b}}$ and $\mathrm{E}=\mathcal{E}_{i_{1} i_{2} \cdots i_{p+q-k}}$ contain $v$ identical real symmetric matrices. Therefore, in this case $\Pi=\mathcal{E}_{\beta_{1} \beta_{2} \cdots \beta_{u}} \mathcal{E}_{i_{1} i_{2} \cdots i_{v}}, \mathrm{E}=$ $\mathcal{E}_{i_{1} i_{2} \cdots i_{v}} \mathcal{E}_{i_{1} i_{2} \cdots i_{l}}$ and

$$
\begin{aligned}
& \text { ПЕ }=(-1)^{\frac{v(v-1)}{2}} \sigma\left(i_{1}\right) \sigma\left(i_{2}\right) \cdots \sigma\left(i_{v}\right) \mathcal{E}_{\beta_{1} \beta_{2} \cdots \beta_{u}} \mathcal{E}_{i_{1} i_{2} \cdots i_{l}}, \\
& \mathrm{E \Pi}=(-1)^{\frac{v(v-1)}{2}+v(u+l)} \sigma\left(i_{1}\right) \sigma\left(i_{2}\right) \cdots \sigma\left(i_{v}\right) \mathcal{E}_{\beta_{1} \beta_{2} \cdots \beta_{u}} \mathcal{E}_{i_{1} i_{2} \cdots i_{l}}
\end{aligned}
$$

that is, in this case $\Pi$ and $\mathrm{E}$ commute at $v(u+l) \equiv 0(\bmod 2)$ and anticommute at $v(u+l) \equiv 1(\bmod 2)$.

It is easy to see that permutation conditions of $\Pi$ with the matrix $C$ of the antiautomorphism $\mathcal{A} \rightarrow \widetilde{\mathcal{A}^{\star}}$ are analogous to the conditions (81)-(84). 
Indeed, at $\Pi=\mathcal{E}_{\alpha_{1} \alpha_{2} \cdots \alpha_{a}}$ and $C=\mathcal{E}_{i_{1} i_{2} \cdots i_{p+q-k}}$ these conditions are analogous to (82), that is, $l(m+v) \equiv 0,1(\bmod 2)$. For the matrices $\Pi=\mathcal{E}_{\alpha_{1} \alpha_{2} \cdots \alpha_{a}}$ and $C=\mathcal{E}_{j_{1} j_{2} \cdots j_{k}}$ we obtain the condition (81), that is, $m(u+l) \equiv 0,1(\bmod 2)$. In turn, for $\Pi=\mathcal{E}_{\beta_{1} \beta_{2} \cdots \beta_{b}}$ and $C=\mathcal{E}_{i_{1} i_{2} \cdots i_{p+q-k}}$ we have the condition (84), that is, $v(u+l) \equiv 0,1(\bmod 2)$. Finally, the matrices $\Pi=\mathcal{E}_{\beta_{1} \beta_{2} \cdots \beta_{b}}$ and $\mathrm{C}=\mathcal{E}_{j_{1} j_{2} \cdots j_{k}}$ correspond to (83) with $u(m+v) \equiv 0,1(\bmod 2)$.

Let us consider now permutation conditions of the matrix $\mathrm{K}$ of $\mathcal{A} \rightarrow \overline{\mathcal{A}^{\star}}$ with other elements of the group $\operatorname{Ext}\left(\mathbb{C}_{n}\right)$. As it has been shown previously, the structure of $\mathrm{K}$ is analogous to the structure of $\Pi$, that is, $\mathrm{K}=\mathcal{E}_{\alpha_{1} \alpha_{2} \cdots \alpha_{a}}$ at $a \equiv 1(\bmod 2)$ and $\mathrm{K}=\mathcal{E}_{\beta_{1} \beta_{2} \cdots \beta_{b}}$ at $b \equiv 0(\bmod 2)$. Therefore, permutation conditions for $\mathrm{K}$ are similar to the conditions for $\Pi$. Permutation conditions between $\mathrm{K}$ and $\Pi$ are defined by the relation (70). Coming to the matrix $\mathrm{S}$ of the pseudoantiautomorphism $\mathcal{A} \rightarrow \overline{\widetilde{\mathcal{A}}}$ we see that permutation conditions between $\mathrm{K}$ and $\mathrm{S}$ are analogous to (171)-(174). Namely, if $\mathrm{K}=\mathcal{E}_{\beta_{1} \beta_{2} \cdots \beta_{b}}$ and $\mathbf{S}=\mathcal{E}_{c_{1} c_{2} \cdots c_{s}}$, then from (74) it follows that $\mathbf{K}$ and $\mathbf{S}$ commute at $v \equiv 0$ $(\bmod 2)$ and anticommute at $v \equiv 1(\bmod 2)$, since in this case $b \equiv 0(\bmod 2)$ and $p+q-k \equiv 0(\bmod 2)$. Analogously, if $\mathrm{K}=\mathcal{E}_{\alpha_{1} \alpha_{2} \cdots \alpha_{b}}$ and $\mathbf{S}=\mathcal{E}_{d_{1} d_{2} \cdots d_{g}}$, then from (72) we find that $\mathrm{K}$ and $\mathrm{S}$ commute at $l \equiv 0(\bmod 2)$ and anticommute at $l \equiv 1(\bmod 2)$, since $a \equiv 1(\bmod 2), p+q-k \equiv 0(\bmod 2)$ for this case. Further, if $\mathrm{K}=\mathcal{E}_{\beta_{1} \beta_{2} \cdots \beta_{b}}$ and $\mathrm{S}=\mathcal{E}_{d_{1} d_{2} \cdots d_{g}}$, then from (73) we find that in this case $\mathrm{K}$ and $\mathrm{S}$ commute at $u \equiv 0(\bmod 2)$ and anticommute at $u \equiv 1$ $(\bmod 2)$, since $b \equiv 0(\bmod 2), k \equiv 1(\bmod 2)$. Finally, if $\mathbf{K}=\mathcal{E}_{\alpha_{1} \alpha_{2} \cdots \alpha_{a}}$ and $\mathrm{S}=\mathcal{E}_{c_{1} c_{2} \cdots c_{s}}$, then from (71) it follows that $\mathrm{K}$ and $\mathrm{S}$ commute at $m \equiv 1(\bmod 2)$ and anticommute at $m \equiv 0(\bmod 2)$, since in this case $a, k \equiv 1(\bmod 2)$.

In like manner we can find permutation conditions of $\mathrm{K}$ with the matrix $\mathrm{F}$ of the pseudoantiautomorphism $\mathcal{A} \rightarrow \overline{\widetilde{\mathcal{A}^{\star}}}$. Indeed, for $\mathrm{K}=\mathcal{E}_{\beta_{1} \beta_{2} \cdots \beta_{b}}$ and $\mathrm{F}=\mathcal{E}_{d_{1} d_{2} \cdots d_{g}}$ from (178) it follows that $\mathrm{K}$ and $\mathrm{F}$ commute at $u \equiv 0(\bmod 2)$ and anticommute at $u \equiv 1(\bmod 2)$, since in this case $b, k \equiv 0(\bmod 2)$. Further, if $\mathrm{K}=\mathcal{E}_{\alpha_{1} \alpha_{2} \cdots \alpha_{a}}$ and $\mathrm{F}=\mathcal{E}_{c_{1} c_{2} \cdots c_{s}}$, then from (76) we find that $\mathrm{K}$ and $\mathrm{F}$ commute at $m \equiv 0(\bmod 2)$ and anticommute at $m \equiv 1(\bmod 2)$, since $a \equiv 1(\bmod 2)$, $k \equiv 0(\bmod 2)$. In turn, for the matrices $\mathrm{K}=\mathcal{E}_{\beta_{1} \beta_{2} \cdots \beta_{b}}$ and $\mathbf{F}=\mathcal{E}_{c_{1} c_{2} \cdots c_{s}}$ from (177) we obtain that $\mathrm{K}$ and $\mathrm{F}$ commute at $v \equiv 0(\bmod 2)$ and anticommute at $v \equiv 1(\bmod 2)$, since $b \equiv 0(\bmod 2), p+q-k \equiv 1(\bmod 2)$. Finally, if $\mathrm{K}=\mathcal{E}_{\alpha_{1} \alpha_{2} \cdots \alpha_{a}}$ and $\mathrm{F}=\mathcal{E}_{d_{1} d_{2} \cdots d_{g}}$, then from (75) it follows that $\mathrm{K}$ and $\mathrm{F}$ commute at $l \equiv 1(\bmod 2)$ and anticommute at $l \equiv 0(\bmod 2)$, since in this case $a, p+q-k \equiv 1(\bmod 2)$.

It is easy to see that in virtue of similarity of the matrices $K$ and $\Pi$, permutation conditions of $\mathrm{K}$ with the elements of $\operatorname{Aut}_{ \pm}\left(C_{p, q}\right)$ are analogous to the conditions for $\Pi$. Indeed, if $K=\mathcal{E}_{\beta_{1} \beta_{2} \cdots \beta_{b}}$, then from (80) it follows 
that $\mathrm{K}$ and $\mathrm{W}$ always commute, since in this case $a, b \equiv 0(\bmod 2)$. In turn, if $\mathrm{K}=\mathcal{E}_{\alpha_{1} \alpha_{2} \cdots \alpha_{a}}$, then from (79) we see that $\mathrm{K}$ and $\mathrm{W}$ always anticommute, since $a, b \equiv 1(\bmod 2)$. Further, if $\mathrm{K}=\mathcal{E}_{\beta_{1} \beta_{2} \cdots \beta_{b}}$ and $\mathrm{E}=\mathcal{E}_{j_{1} j_{2} \cdots j_{k}}$, then from (83) it follows that $\mathrm{K}$ and $\mathrm{E}$ commute at $u(m+v) \equiv 0(\bmod 2)$ and anticommute at $u(m+v) \equiv 1(\bmod 2)$. For the matrices $\mathrm{K}=\mathcal{E}_{\alpha_{1} \alpha_{2} \cdots \alpha_{a}}$ and $\mathrm{E}=\mathcal{E}_{j_{1} j_{2} \cdots j_{k}}$ from (81) it follows $m(u+l) \equiv 0,1(\bmod 2)$. Correspondingly, for the matrices $\mathrm{K}=\mathcal{E}_{\beta_{1} \beta_{2} \cdots \beta_{b}}$ and $\mathrm{E}=\mathcal{E}_{i_{1} i_{2} \cdots i_{p+q-k}}$ from (84) we obtain the condition $v(u+l) \equiv 0,1(\bmod 2)$. For $\mathrm{K}=\mathcal{E}_{\alpha_{1} \alpha_{2} \cdots \alpha_{a}}$ and $\mathrm{E}=\mathcal{E}_{i_{1} i_{2} \cdots i_{p+q}-k}$ from (82) we have $l(m+v) \equiv 0,1(\bmod 2)$. In turn, for $\mathrm{K}=\mathcal{E}_{\beta_{1} \beta_{2} \cdots \beta_{b}}$ and $\mathrm{C}=$ $\mathcal{E}_{i_{1} i_{2} \cdots i_{p+q-k}}$ from (84) we obtain $v(u+l) \equiv 0,1(\bmod 2)$, for $\mathrm{K}=\mathcal{E}_{\alpha_{1} \alpha_{2} \cdots \alpha_{a}}$ and $\mathrm{C}=\mathcal{E}_{i_{1} i_{2} \cdots i_{p+q-k}}$ from (82) we obtain $l(m+v) \equiv 0,1(\bmod 2)$, for $\mathrm{K}=\mathcal{E}_{\beta_{1} \beta_{2} \cdots \beta_{b}}$ and $C=\mathcal{E}_{j_{1} j_{2} \cdots j_{k}}$ from (83) it follows $u(m+v) \equiv 0,1(\bmod 2)$ and, finally, for $\mathrm{K}=\mathcal{E}_{\alpha_{1} \alpha_{2} \cdots \alpha_{a}}$ and $\mathrm{C}=\mathcal{E}_{j_{1} j_{2} \cdots j_{k}}$ from (81) we have $m(u+l) \equiv 0,1(\bmod 2)$.

Let consider permutation conditions of the matrix $S$ of the transformation $\mathcal{A} \rightarrow \overline{\widetilde{A}}$ with other elements of the group $\operatorname{Ext}\left(\mathbb{C}_{n}\right)$. Permutation conditions of $S$ with the matrices $\Pi$ and $K$ have been defined previously (see (71)-(174)). Now we define permutation conditions of $S$ with the matrix $F$ of the pseudoantiautomorphism $\mathcal{A} \rightarrow \widetilde{\widetilde{\mathcal{A}^{\star}}}$ and elements of the subgroup $\operatorname{Aut}_{ \pm}\left(C_{p, q}\right)$. So, let $\mathrm{S}=\mathcal{E}_{c_{1} c_{2} \cdots c_{s}}$ and $\mathrm{F}=\mathcal{E}_{d_{1} d_{2} \cdots d_{g}}$, where $s \equiv 0(\bmod 2)$ and $g \equiv 0(\bmod 2)$. As known, in this case the product $S$ contains all complex symmetric matrices and all real skewsymmetric matrices of the spinbasis. In turn, the product $F$ contains all complex skewsymmetric and real symmetric matrices. Therefore, the product SF does not contain identical matrices. Then

$$
\mathrm{SF}=(-1)^{s g} \mathrm{FS}
$$

that is, in this case $\mathrm{S}$ and $\mathrm{F}$ always commute, since $s, g \equiv 0(\bmod 2)$. If $\mathrm{S}=\mathcal{E}_{d_{1} d_{2} \cdots d_{g}}$ and $\mathrm{F}=\mathcal{E}_{c_{1} c_{2} \cdots c_{s}}$, where $s, g \equiv 1(\bmod 2)$, then from (85) it follows that $S$ and $F$ always anticommute.

Further, if $\mathrm{S}=\mathcal{E}_{c_{1} c_{2} \cdots c_{s}}$, then permutation conditions of $\mathrm{S}$ with the matrix W of the automorphism $\mathcal{A} \rightarrow \mathcal{A}^{\star}$ have the form:

$$
\begin{aligned}
\mathrm{SW} & =(-1)^{\frac{s(s-1)}{2}} \sigma\left(c_{1}\right) \sigma\left(c_{2}\right) \cdots \sigma\left(c_{s}\right) \mathcal{E}_{d_{1} d_{2} \cdots d_{g}}, \\
\mathrm{WS} & =(-1)^{\frac{s(s-1)}{2}+s g} \sigma\left(c_{1}\right) \sigma\left(c_{2}\right) \cdots \sigma\left(c_{s}\right) \mathcal{E}_{d_{1} d_{2} \cdots d_{g}}
\end{aligned}
$$

that is, in this case $\mathrm{S}$ always commutes with $\mathrm{W}$, since $s, g \equiv 0(\bmod 2)$. In turn, the matrix $\mathrm{S}=\mathcal{E}_{d_{1} d_{2} \cdots d_{g}}$ always anticommutes with $\mathrm{W}$, since $s, g \equiv 1$ $(\bmod 2)$.

Let us define now permutation conditions between $\mathrm{S}$ and $\mathrm{E}$. If $\mathrm{S}=\mathcal{E}_{c_{1} c_{2} \cdots c_{s}}$ and $\mathrm{E}=\mathcal{E}_{j_{1} j_{2} \cdots j_{k}}$, then the product SE contains $u$ identical real skewsymmetric 
matrices. Hence it follows that $S=\mathcal{E}_{c_{1} c_{2} \cdots c_{l}} \mathcal{E}_{i_{1} i_{2} \cdots i_{u}}$ and $\mathrm{E}=\mathcal{E}_{i_{1} i_{2} \cdots i_{u}} \mathcal{E}_{j_{1} j_{2} \cdots j_{m}}$. Then

$$
\begin{aligned}
\mathrm{SE} & =(-1)^{\frac{u(u-1)}{2}} \sigma\left(i_{1}\right) \sigma\left(i_{2}\right) \cdots \sigma\left(i_{u}\right) \mathcal{E}_{c_{1} c_{2} \cdots c_{l}} \mathcal{E}_{j_{1} j_{2} \cdots j_{m}}, \\
\mathrm{ES} & =(-1)^{\frac{u(u-1)}{2}+u(l+m)} \sigma\left(i_{1}\right) \sigma\left(i_{2}\right) \cdots \sigma\left(i_{u}\right) \mathcal{E}_{c_{1} c_{2} \cdots c_{l}} \mathcal{E}_{j_{1} j_{2} \cdots j_{m}}
\end{aligned}
$$

that is, $\mathrm{S}$ and $\mathrm{E}$ commute at $u(l+m) \equiv 0(\bmod 2)$ and anticommute at $u(l+m) \equiv 1(\bmod 2)$.

In turn, the products $S=\mathcal{E}_{d_{1} d_{2} \cdots d_{g}}$ and $\mathbf{E}=\mathcal{E}_{j_{1 j_{2} \cdots j_{k}}}$ contain $m$ identical complex skewsymmetric matrices. Therefore, $\mathbf{S}=\mathcal{E}_{d_{1} d_{2} \cdots d_{v}} \mathcal{E}_{i_{1} i_{2} \cdots i_{m}}$, $\mathrm{E}=\mathcal{E}_{i_{1} i_{2} \cdots i_{m}} \mathcal{E}_{j_{1} j_{2} \cdots j_{u}}$ and

$$
\begin{aligned}
\mathrm{SE} & =(-1)^{\frac{m(m-1)}{2}} \sigma\left(i_{1}\right) \sigma\left(i_{2}\right) \cdots \sigma\left(i_{m}\right) \mathcal{E}_{d_{1} d_{2} \cdots d_{v}} \mathcal{E}_{j_{1} j_{2} \cdots j_{u}} \\
\mathrm{ES} & =(-1)^{\frac{m(m-1)}{2}+m(v+u)} \sigma\left(i_{1}\right) \sigma\left(i_{2}\right) \cdots \sigma\left(i_{m}\right) \mathcal{E}_{d_{1} d_{2} \cdots d_{v}} \mathcal{E}_{j_{1} j_{2} \cdots j_{u}}
\end{aligned}
$$

that is, permutation conditions between $S$ and $E$ in this case have the form $m(v+u) \equiv 0,1(\bmod 2)$.

Further, the products $\mathrm{S}=\mathcal{E}_{d_{1} d_{2} \cdots d_{g}}$ and $\mathrm{E}=\mathcal{E}_{i_{1} i_{2} \cdots i_{p+q-k}}$ contain $v$ identical real symmetric matrices. Then $\mathrm{S}=\mathcal{E}_{d_{1} d_{2} \cdots d_{m}} \mathcal{E}_{i_{1} i_{2} \cdots i_{v}}, \mathrm{E}=\mathcal{E}_{i_{1} i_{2} \cdots i_{v}} \mathcal{E}_{i_{1} i_{2} \cdots i_{l}}$ and

$$
\begin{aligned}
\mathrm{SE} & =(-1)^{\frac{v(v-1)}{2}} \sigma\left(i_{1}\right) \sigma\left(i_{2}\right) \cdots \sigma\left(i_{v}\right) \mathcal{E}_{d_{1} d_{2} \cdots d_{m}} \mathcal{E}_{i_{1} i_{2} \cdots i_{l}}, \\
\mathrm{ES} & =(-1)^{\frac{v(v-1)}{2}+v(m+l)} \sigma\left(i_{1}\right) \sigma\left(i_{2}\right) \cdots \sigma\left(i_{v}\right) \mathcal{E}_{d_{1} d_{2} \cdots d_{m}} \mathcal{E}_{i_{1} i_{2} \cdots i_{l}}
\end{aligned}
$$

that is, permutation conditions between $\mathrm{S}$ and $\mathrm{E}$ are $v(m+l) \equiv 0,1(\bmod 2)$.

Finally, the products $\mathrm{S}=\mathcal{E}_{c_{1} c_{2} \cdots c_{S}}$ and $\mathrm{E}=\mathcal{E}_{i_{1} i_{2} \cdots i_{p+q-k}}$ contain $l$ identical complex symmetric matrices and, therefore, $S=\mathcal{E}_{c_{1} c_{2} \cdots c_{u}} \mathcal{E}_{i_{1} i_{2} \cdots i_{l}}, \mathrm{E}=$ $\mathcal{E}_{i_{1} i_{2} \cdots i_{l}} \mathcal{E}_{i_{1} i_{2} \cdots i_{v}}$. Then

$$
\begin{aligned}
& \mathrm{SE}=(-1)^{\frac{l(l-1)}{2}} \sigma\left(i_{1}\right) \sigma\left(i_{2}\right) \cdots \sigma\left(i_{l}\right) \mathcal{E}_{c_{1} c_{2} \cdots c_{u}} \mathcal{E}_{i_{1} i_{2} \cdots i_{v}} \\
& \mathrm{ES}=(-1)^{\frac{l(l-1)}{2}+l(u+v)} \sigma\left(i_{1}\right) \sigma\left(i_{2}\right) \cdots \sigma\left(i_{l}\right) \mathcal{E}_{c_{1} c_{2} \cdots c_{u}} \mathcal{E}_{i_{1} i_{2} \cdots i_{v}}
\end{aligned}
$$

and permutation conditions for $\mathrm{S}$ and $\mathrm{E}$ in this case have the form $l(u+v) \equiv 0,1$ $(\bmod 2)$.

It is easy to see that permutation conditions between $S$ and $C$ are analogous to (87)- (90). Indeed, if $S=\mathcal{E}_{c_{1} c_{2} \cdots c_{s}}$ and $C=\mathcal{E}_{i_{1} i_{2} \cdots i_{p+q-k}}$, then from (90) it follows the comparison $l(u+v) \equiv 0,1(\bmod 2)$. In turn, if $\mathbf{S}=\mathcal{E}_{d_{1} d_{2} \cdots d_{g}}$ and $\mathrm{C}=\mathcal{E}_{i_{1} i_{2} \cdots i_{p+q-k}}$, then from (89) we obtain $v(m+l) \equiv 0,1(\bmod 2)$. Further, 
for $\mathbf{S}=\mathcal{E}_{d_{1} d_{2} \cdots d_{g}}$ and $\mathrm{C}=\mathcal{E}_{j_{1} j_{2} \cdots j_{k}}$ from (88) it follows that $m(v+u) \equiv 0,1$ $(\bmod 2)$. Analogously, for $S=\mathcal{E}_{c_{1} c_{2} \cdots c_{s}}$ and $C=\mathcal{E}_{j_{1} j_{2} \cdots j_{k}}$ from (87) we have $u(l+m) \equiv 0,1(\bmod 2)$.

Finally, let us consider permutation conditions of the matrix $\mathrm{F}$ of $\mathcal{A} \rightarrow \overline{\widetilde{\mathcal{A}^{\star}}}$ with other elements of the group $\operatorname{Ext}\left(\mathbb{C}_{n}\right)$. Permutation conditions of $\mathrm{F}$ with the matrices $\Pi, \mathrm{K}$ and $\mathrm{S}$ have been found previously (see (75)-(178), (85)). Now we define permutation conditions between $\mathrm{F}$ and the elements of the subgroups Aut $_{ \pm}\left(C \ell_{p, q}\right)$. It is easy to see that permutation conditions between $\mathrm{F}$ and $\mathrm{W}$ are equivalent to (86), that is, $\mathrm{F}=\mathcal{E}_{d_{1} d_{2} \cdots d_{g}}$ always commute with $\mathbf{W}$, since $s, g \equiv 0(\bmod 2)$, and $\mathrm{F}=\mathcal{E}_{c_{1} c_{2} \cdots c_{s}}$ always anticommute with $\mathrm{W}$, since in this case $s, g \equiv 1(\bmod 2)$. In turn, permutation conditions between $\mathrm{F}$ and $\mathrm{E}$ are equivalent to (87)-(90). Indeed, if $F=\mathcal{E}_{d_{1} d_{2} \cdots d_{g}}$ and $\mathrm{E}=\mathcal{E}_{j_{1} j_{2} \cdots j_{k}}$, then from (88) it follows the comparison $m(v+u) \equiv 0,1(\bmod 2)$. For $\mathrm{F}=\mathcal{E}_{c_{1} c_{2} \cdots c_{s}}$ and $\mathrm{E}=\mathcal{E}_{j_{1} j_{2} \cdots j_{k}}$ from (87) we have $u(l+m) \equiv 0,1(\bmod 2)$. Analogously, for $\mathrm{F}=\mathcal{E}_{c_{1} c_{2} \cdots c_{s}}$ and $\mathrm{E}=\mathcal{E}_{i_{1} i_{2} \cdots i_{p+q-k}}$ from (90) we obtain $l(u+v) \equiv 0,1(\bmod 2)$, and for $\mathrm{F}=\mathcal{E}_{d_{1} d_{2} \cdots d_{g}}, \mathrm{E}=\mathcal{E}_{i_{1} i_{2} \cdots i_{p+q-k}}$ from (89) it follows $v(m+l) \equiv 0,1$ $(\bmod 2)$. It is easy to see that permutation conditions between $F$ and $C$ are equivalent to (87)-(90). Namely, for $\mathrm{F}=\mathcal{E}_{d_{1} d_{2} \cdots d_{g}}, \mathrm{C}=\mathcal{E}_{i_{1} i_{2} \cdots i_{p+q-k}}$ from (89) we obtain $v(m+l) \equiv 0,1(\bmod 2)$, for $\mathrm{F}=\mathcal{E}_{c_{1} c_{2} \cdots c_{s}}, \mathrm{C}=\mathcal{E}_{i_{1} i_{2} \cdots i_{p+q-k}}$ from (90) it follows $l(u+v) \equiv 0,1(\bmod 2)$. Correspondingly, for $\mathrm{F}=\mathcal{E}_{c_{1} c_{2} \cdots c_{s}}$, $\mathrm{C}=\mathcal{E}_{j_{1 j_{2} \cdots j_{k}}}$ from (87) we find $u(l+m) \equiv 0,1(\bmod 2)$, and for $\mathrm{F}=\mathcal{E}_{d_{1} d_{2} \cdots d_{g}}$, $\mathrm{C}=\mathcal{E}_{j_{1} j_{2} \cdots j_{k}}$ from (88) we see that $m(v+u) \equiv 0,1(\bmod 2)$.

Now, we are in a position to define a detailed classification for extended automorphism groups $\operatorname{Ext}\left(\mathbb{C}_{n}\right)$. First of all, since for the subalgebras $C \ell_{p, q}$ over the ring $\mathbb{K} \simeq \mathbb{R}$ the group $\operatorname{Ext}\left(\mathbb{C}_{n}\right)$ is reduced to Aut ${ }_{ \pm}\left(\mathbb{C}_{n}\right)$, then all essentially different groups $\operatorname{Ext}\left(\mathbb{C}_{n}\right)$ correspond to the subalgebras $C \ell_{p, q}$ with the quaternionic ring $\mathbb{K} \simeq \mathbb{H}, p-q \equiv 4,6(\bmod 8)$. Let us classify the groups $\operatorname{Ext}\left(\mathbb{C}_{n}\right)$ with respect to their subgroups $\operatorname{Aut}_{ \pm}\left(C \ell_{p, q}\right)$. Taking into account the structure of $\operatorname{Aut}_{ \pm}\left(C_{p, q}\right)$ at $p-q \equiv 4,6(\bmod 8)$ (see Theorem 5) we obtain for the group $\operatorname{Ext}\left(\mathbb{C}_{n}\right)=\{\mathrm{I}, \mathrm{W}, \mathrm{E}, \mathrm{C}, \Pi, \mathrm{K}, \mathrm{S}, \mathrm{F}\}$ the following possible realizations:

$$
\begin{aligned}
& \text { Ext }^{1}=\left\{1, \mathcal{E}_{12 \cdots p+q}, \mathcal{E}_{j_{1} j_{2} \cdots j_{k}}, \mathcal{E}_{i_{1} i_{2} \cdots i_{p+q}}, \mathcal{E}_{\alpha_{1} \alpha_{2} \cdots \alpha_{a}}, \mathcal{E}_{\beta_{1} \beta_{2} \cdots \beta_{b}}, \mathcal{E}_{c_{1} c_{2} \cdots c_{s}}, \mathcal{E}_{d_{1} d_{2} \cdots d_{g}}\right\}, \\
& \text { Ext }^{2}=\left\{1, \mathcal{E}_{12 \cdots p+q}, \mathcal{E}_{j_{1} j_{2} \cdots j_{k}}, \mathcal{E}_{i_{1} i_{2} \cdots i_{p+q-k}}, \mathcal{E}_{\beta_{1} \beta_{2} \cdots \beta_{b}}, \mathcal{E}_{\alpha_{1} \alpha_{2} \cdots \alpha_{a}}, \mathcal{E}_{d_{1} d_{2} \cdots d_{g}}, \mathcal{E}_{c_{1} c_{2} \cdots c_{s}}\right\}, \\
& \text { Ext }^{3}=\left\{I, \mathcal{E}_{12 \cdots p+q}, \mathcal{E}_{i_{1} i_{2} \cdots i_{p+q}-k}, \mathcal{E}_{j_{1} j_{2} \cdots j_{k}}, \mathcal{E}_{\alpha_{1} \alpha_{2} \cdots \alpha_{a}}, \mathcal{E}_{\beta_{1} \beta_{2} \cdots \beta_{b}}, \mathcal{E}_{d_{1} d_{2} \cdots d_{g}}, \mathcal{E}_{c_{1} c_{2} \cdots c_{s}}\right\}, \\
& \text { Ext }^{4}=\left\{I, \mathcal{E}_{12 \cdots p+q}, \mathcal{E}_{i_{1} i_{2} \cdots i_{p+q}-k}, \mathcal{E}_{j_{1} j_{2} \cdots j_{k}}, \mathcal{E}_{\beta_{1} \beta_{2} \cdots \beta_{b}}, \mathcal{E}_{\alpha_{1} \alpha_{2} \cdots \alpha_{a}}, \mathcal{E}_{c_{1} c_{2} \cdots c_{s}}, \mathcal{E}_{d_{1} d_{2} \cdots d_{g}}\right\} .
\end{aligned}
$$

The groups $\operatorname{Ext}^{1}\left(\mathbb{C}_{n}\right)$ and $\operatorname{Ext}^{2}\left(\mathbb{C}_{n}\right)$ have Abelian subgroups Aut $\left(C \ell_{p, q}\right)\left(\mathbb{Z}_{2} \otimes\right.$ $\mathbb{Z}_{2}$ or $\left.\mathbb{Z}_{4}\right)$. In turn, the groups $\operatorname{Ext}^{3}\left(\mathbb{C}_{n}\right)$ and Ext ${ }^{4}\left(\mathbb{C}_{n}\right)$ have only non-Abelian subgroups Aut A $_{+}\left(C \ell_{p, q}\right)\left(Q_{4} / \mathbb{Z}_{2}\right.$ or $\left.D_{4} / \mathbb{Z}_{2}\right)$. 
Let us start with the group $\operatorname{Ext}^{1}\left(\mathbb{C}_{n}\right)$. All the elements of $\operatorname{Ext}^{1}\left(\mathbb{C}_{n}\right)$ are even products, that is, $p+q \equiv 0(\bmod 2), k \equiv 0(\bmod 2), a \equiv 0(\bmod 2), b \equiv 0$ $(\bmod 2), s \equiv 0(\bmod 2)$ and $g \equiv 0(\bmod 2)$. At this point, the elements I, W, $\mathrm{E}, \mathrm{C}$ form Abelian subgroups $\mathbb{Z}_{2} \otimes \mathbb{Z}_{2}$ or $\mathbb{Z}_{4}$ (see Theorem 5 ). In virtue of (70) the element $\Pi$ commutes with $\mathrm{K}$, and from (71) it follows that $\Pi$ commutes with $\mathrm{S}$ at $m \equiv 0(\bmod 2)$. From $(75)$ we see that $\Pi$ commutes with $\mathrm{F}$ at $l \equiv 0$ $(\bmod 2)$, and from (79) it follows that $\Pi$ commutes with W. The conditions (81) show that $\Pi$ commutes with $\mathrm{E}$ at $m(u+l) \equiv 0(\bmod 2)$ and commutes with $\mathrm{C}$ at $l(m+v) \equiv 0(\bmod 2)$. Further, the element $\mathrm{K}$ commutes with $\mathrm{S}$ at $v \equiv 0(\bmod 2)$ and commutes with $\mathrm{F}$ at $u \equiv 0(\bmod 2)$. And also $\mathrm{K} \in \mathrm{Ext}^{1}$ always commutes with $\mathrm{W}$ and commutes correspondingly with $\mathrm{E}$ and $\mathrm{C}$ at $u(m+v) \equiv 0(\bmod 2)$ and $v(u+l) \equiv 0(\bmod 2)$. From $(85)$ and $(86)$ it follows that the element $\mathrm{S}$ always commutes with $\mathrm{F}$ and $\mathrm{W}$. The conditions (87) and (90) show that $\mathrm{S}$ commutes with $\mathrm{E}$ and $\mathrm{C}$ correspondingly at $u(l+m) \equiv 0$ $(\bmod 2)$ and $l(u+v) \equiv 0(\bmod 2)$. In virtue of $(86)$ the element $\mathrm{F}$ always commutes with W. Finally, from (88) and (89) it follows that F commutes with $\mathrm{E}$ and $\mathrm{C}$ correspondingly at $m(v+u) \equiv 0(\bmod 2)$ and $v(m+l) \equiv 0(\bmod 2)$. Thus, the group $\operatorname{Ext}^{1}\left(\mathbb{C}_{n}\right)$ is Abelian at $m, l, u, v \equiv 0(\bmod 2)$. In case of the subgroup Aut $-\left(C \ell_{p, q}\right) \simeq \mathbb{Z}_{2} \otimes \mathbb{Z}_{2}(p-q \equiv 4(\bmod 8))$ we obtain an Abelian group $\operatorname{Ext}_{-}^{1}\left(\mathbb{C}_{n}\right) \simeq \mathbb{Z}_{2} \otimes \mathbb{Z}_{2} \otimes \mathbb{Z}_{2}$ with the signature $(+,+,+,+,+,+,+)$ for the elements $\Pi, \mathrm{K}, \mathrm{S}$ and $\mathrm{F}$ with positive squares $(m-l \equiv 0,4(\bmod 8), v-u \equiv 0,4$ $(\bmod 8), u+l \equiv 0,4(\bmod 8)$ and $m+v \equiv 0,4(\bmod 8))$. It is easy to see that for the type $p-q \equiv 4(\bmod 8)$ there exists also $\operatorname{Ext}_{-}^{1}\left(\mathbb{C}_{n}\right) \simeq \mathbb{Z}_{4} \otimes \mathbb{Z}_{2}$ with the signature $(+,+,+,-,-,-,-)$ and the subgroup $\mathbb{Z}_{2} \otimes \mathbb{Z}_{2}$, where $m-l \equiv$ $2,6(\bmod 8), v-u \equiv 2,6(\bmod 8), u+l \equiv 2,6(\bmod 8)$ and $m+v \equiv 2,6$ $(\bmod 8)$. Further, for the type $p-q \equiv 4(\bmod 8)$ there exist Abelian groups $\operatorname{Ext}_{-}^{1}\left(\mathbb{C}_{n}\right) \simeq \mathbb{Z}_{4} \otimes \mathbb{Z}_{2}$ with the signatures $(+,-,-, d, e, f, g)$ and subgroups $\mathbb{Z}_{4}$, where among the symbols $d, e, f, g$ there are two pluses and two minuses. Correspondingly, at $m, v, l, u \equiv 0(\bmod 2)$ for the type $p-q \equiv 6(\bmod 8)$ there exist Abelian groups $\operatorname{Ext}_{-}^{1}\left(\mathbb{C}_{n}\right) \simeq \mathbb{Z}_{4} \otimes \mathbb{Z}_{2}$ with the signatures $(-,+,-, d, e, f, g)$ and $(-,-,+, d, e, f, g)$ if $m-u \equiv 0,1,4,5(\bmod 8), v-l \equiv 2,3,6,7(\bmod 8)$ and $m-u \equiv 2,3,6,7(\bmod 8), v-l \equiv 0,1,4,5(\bmod 8)$.

It is easy to see that from the comparison $k, a, b, s, g \equiv 0(\bmod 2)$ it follows that the numbers $m, v, l, u$ are simultaneously even or odd. The case $m, v, l, u \equiv 0(\bmod 2)$, considered previously, leads to the Abelian groups $\mathrm{Ext}_{-}^{1}$. In contrast to this, the case $m, v, l, u \equiv 1(\bmod 2)$ leads to non-Abelian groups Ext ${ }_{+}^{1}$ with Abelian subgroups $\mathbb{Z}_{4}$ and $\mathbb{Z}_{2} \otimes \mathbb{Z}_{2}$ (it follows from (70)-(171)). Namely, in this case we have the group Ext ${ }_{+}^{1} \simeq \mathbb{Z}_{4}^{*} \otimes \mathbb{Z}_{2}$ with the subgroup $\mathbb{Z}_{4}$ for $p-q \equiv 4,6(\bmod 8)$ (it should be noted that signatures of the groups 
$\mathbb{Z}_{4} \otimes \mathbb{Z}_{2}$ and $\stackrel{*}{\mathbb{Z}}_{4} \otimes \mathbb{Z}_{2}$ do not coincide), the group Ext ${ }_{+}^{1} \simeq Q_{4}$ with the subgroup $\mathbb{Z}_{4}$ for $p-q \equiv 4,6(\bmod 8)$ and $\mathrm{Ext}_{+}^{1} \simeq D_{4}$ with $\mathbb{Z}_{2} \otimes \mathbb{Z}_{2}$ for the type $p-q \equiv 4$ $(\bmod 8)$.

Let us consider now the group $\operatorname{Ext}^{2}\left(\mathbb{C}_{n}\right)$. In this case among the elements of Ext ${ }^{2}$ there are both even and odd elements: $k \equiv 0(\bmod 2), b \equiv 1(\bmod 2)$, $a \equiv 1(\bmod 2), g \equiv 1(\bmod 2)$ and $s \equiv 1(\bmod 2)$. At this point, the elements I, W, E, C form Abelian subgroups $\mathbb{Z}_{2} \otimes \mathbb{Z}_{2}$ and $\mathbb{Z}_{4}$. In virtue of (170) the element $\Pi$ always anticommutes with $\mathrm{K}$, and from (85) it follows that the elements $\mathrm{S}$ and $\mathrm{F}$ always anticommute. Therefore, all the groups Ext ${ }^{2}$ are non-Abelian. Among these groups there are the following isomorphisms: $\mathrm{Ext}_{+}^{2} \simeq \stackrel{*}{\mathbb{Z}}_{4} \otimes \mathbb{Z}_{2}$ with the signatures $(+,-,-, d, e, f, g)$ for the type $p-q \equiv 4(\bmod 8)$ and $(-,+,-, d, e, f, g),(-,-,+, d, e, f, g)$ for $p-q \equiv 6(\bmod 8)$, where among the symbols $d, e, f, g$ there are two pluses and two minuses; Ext ${ }_{+}^{2} \simeq Q_{4}$ with $(+,-,-,-,-,-,-)$ for $p-q \equiv 4(\bmod 8)$ and $(-,+,-,-,-,-,-)$, $(-,-,+,-,-,-,-)$ for $p-q \equiv 6(\bmod 8) ; \operatorname{Ext}_{+}^{2} \simeq D_{4}$ with $(a, b, c,+,+,+,+)$ and $(+,+,+, d, e, f, g)$, where among $a, b, c$ there are two minuses and one plus, and among $d, e, f, g$ there are two pluses and two minuses. For all the groups Ext ${ }^{2}$ among the numbers $m, v, u, l$ there are both even and odd numbers.

Let consider the group $\operatorname{Ext}^{3}\left(\mathbb{C}_{n}\right)$. First of all, the groups Ext ${ }^{3}$ contain nonAbelian subgroups $\operatorname{Aut}_{+}\left(C \ell_{p, q}\right)$ (the elements $\mathrm{E}$ and $\mathrm{C}$ are odd). Therefore, all the groups $\mathrm{Ext}^{3}$ are non-Abelian. Among these groups there are the following isomorphisms: $\mathrm{Ext}_{+}^{3} \simeq D_{4}$ with $(+,-,+, d, e, f, g)$ and $(+,+,-, d, e, f, g)$ for the type $p-q \equiv 4(\bmod 8)$, where among $d, e, f, g$ there are three pluses and one minus; $\mathrm{Ext}_{+}^{3} \simeq Q_{4}$ with $(-,-,-, d, e, f, g)$, where among $d, e, f, g$ there are one plus and three minuses; Ext $_{+}^{3} \simeq D_{4}$ with $(-,+,+, d, e, f, g)$, where among $d, e, f, g$ there are three pluses and one minus (the type $p-q \equiv 6$ $(\bmod 8))$. Besides, there exist the groups Ext ${ }_{+}^{3} \simeq \stackrel{\mathbb{Z}}{4}_{4} \otimes \mathbb{Z}_{2}$ with the signatures $(+,-,+, d, e, f, g),(+,+,-, d, e, f, g)$ for the type $p-q \equiv 4(\bmod 8)$ and $(-,-,-, d, e, f, g),(-,+,+, d, e, f, g)$ for $p-q \equiv 6(\bmod 8)$, where among $d$, $e, f, g$ there are one plus and three minuses.

Finally, let us consider the group $\operatorname{Ext}^{4}\left(\mathbb{C}_{n}\right)$. These groups contain nonAbelian subgroups Aut ${ }_{+}\left(C_{p, q}\right)$ and, therefore, all Ext ${ }^{4}$ are non-Abelian. The isomorphism structure of Ext ${ }^{4}$ is similar to Ext ${ }^{3}$.

It is easy to see that a full number of all possible signatures $(a, b, c, d, e, f, g)$ is equal to $2^{7}=128$. At this point, we have eight signature types: (seven ' + '), (one '-', six '+'), (two '-', five '+'), (three '-', four '+'), (four '-', three '+'), (five '-', two '+'), (six '-', one '+'), (seven '-'). However, only four types from enumerated above correspond to finite groups of order 8: (seven 
'+') $\rightarrow \mathbb{Z}_{2} \otimes \mathbb{Z}_{2} \otimes \mathbb{Z}_{2}$, (two '-', five '+') $\rightarrow D_{4}$, (four '-', three '+') $\rightarrow \mathbb{Z}_{4} \otimes \mathbb{Z}_{2}$ $\left(\mathbb{Z}_{4}^{*} \otimes \mathbb{Z}_{2}\right)$ and (six '-', one ' + ') $\rightarrow Q_{4}$. Therefore, for the group Ext there exist 64 different realizations.

Example 2. Let us study an extended automorphism group of the Dirac algebra $\mathbb{C}_{4}$. We evolve in $\mathbb{C}_{4}$ the real subalgebra with the quaternionic ring. Let it be the spacetime algebra $C_{p, q}$ with a spinbasis defined by the matrices (67). We define now elements of the group $\operatorname{Ext}\left(\mathbb{C}_{4}\right)$. First of all, the matrix of the automorphism $\mathcal{A} \rightarrow \mathcal{A}^{\star}$ has a form: $\mathbf{W}=\gamma_{0} \gamma_{1} \gamma_{2} \gamma_{3}$. Further, since

$$
\gamma_{0}^{\top}=\gamma_{0}, \quad \gamma_{1}^{\top}=-\gamma_{1}, \quad \gamma_{2}^{\top}=-\gamma_{2}, \quad \gamma_{3}^{\top}=-\gamma_{3},
$$

then in accordance with Theorem $[5$ the matrix $\mathrm{E}$ of the antiautomorphism $\mathcal{A} \rightarrow \widetilde{\mathcal{A}}$ is an even product of skewsymmetric matrices of the spinbasis (67), that is, $\mathrm{E}=\gamma_{1} \gamma_{3}$. From the definition $\mathrm{C}=\mathrm{EW}$ we find that the matrix of the antiautomorphism $\mathcal{A} \rightarrow \widetilde{\mathcal{A}^{\star}}$ has a form $\mathrm{C}=\gamma_{0} \gamma_{2} \cdot \gamma$-basis contains three real matrices $\gamma_{0}, \gamma_{1}$ and $\gamma_{3}$, therefore, for the matrix of the pseudoautomorphism $\mathcal{A} \rightarrow \overline{\mathcal{A}}$ we obtain $\Pi=\gamma_{0} \gamma_{1} \gamma_{3}$ (see Theorem 3). Further, in accordance with $\mathrm{K}=\Pi \mathrm{W}$ for the matrix of the pseudoautomorphism $\mathcal{A} \rightarrow \overline{\mathcal{A}^{\star}}$ we have $\mathrm{K}=\gamma_{2}$. Finally, for the pseudoantiautomorphisms $\mathcal{A} \rightarrow \overline{\widetilde{\mathcal{A}}}, \mathcal{A} \rightarrow \overline{\widetilde{\mathcal{A}}}$ from the definitions $S=\Pi E, F=\Pi C$ it follows $S=\gamma_{0}, F=\gamma_{1} \gamma_{2} \gamma_{3}$. Thus, we come to the following extended automorphism group:

$$
\begin{aligned}
\operatorname{Ext}\left(\mathbb{C}_{4}\right) \simeq\{\mathrm{I}, \mathrm{W}, \mathrm{E}, \mathrm{C}, \mathrm{I}, \mathrm{K}, \mathrm{S}, \mathrm{F}\} \simeq \\
\\
\left\{\mathrm{II}, \gamma_{0} \gamma_{1} \gamma_{2} \gamma_{3}, \gamma_{1} \gamma_{3}, \gamma_{0} \gamma_{2}, \gamma_{0} \gamma_{1} \gamma_{3}, \gamma_{2}, \gamma_{0}, \gamma_{1} \gamma_{2} \gamma_{3}\right\}
\end{aligned}
$$

The multiplication table of this group has a form:

\begin{tabular}{|c||c|c|c|c|c|c|c|c|}
\hline & $\mathrm{I}$ & $\gamma_{0123}$ & $\gamma_{13}$ & $\gamma_{02}$ & $\gamma_{013}$ & $\gamma_{2}$ & $\gamma_{0}$ & $\gamma_{123}$ \\
\hline \hline $\mathrm{I}$ & $\mathrm{I}$ & $\gamma_{0123}$ & $\gamma_{13}$ & $\gamma_{02}$ & $\gamma_{013}$ & $\gamma_{2}$ & $\gamma_{0}$ & $\gamma_{123}$ \\
\hline$\gamma_{0123}$ & $\gamma_{0123}$ & $-\mathrm{I}$ & $\gamma_{02}$ & $-\gamma_{012}$ & $-\gamma_{2}$ & $\gamma_{013}$ & $-\gamma_{123}$ & $\gamma_{0}$ \\
\hline$\gamma_{13}$ & $\gamma_{13}$ & $\gamma_{02}$ & $-\mathrm{I}$ & $-\gamma_{0123}$ & $-\gamma_{0}$ & $-\gamma_{123}$ & $\gamma_{013}$ & $\gamma_{2}$ \\
\hline$\gamma_{02}$ & $\gamma_{02}$ & $-\gamma_{13}$ & $-\gamma_{0123}$ & $\mathrm{I}$ & $\gamma_{123}$ & $-\gamma_{0}$ & $-\gamma_{2}$ & $\gamma_{013}$ \\
\hline$\gamma_{013}$ & $\gamma_{013}$ & $\gamma_{2}$ & $-\gamma_{0}$ & $-\gamma_{123}$ & $-\mathrm{I}$ & $-\gamma_{0123}$ & $\gamma_{13}$ & $\gamma_{02}$ \\
\hline$\gamma_{2}$ & $\gamma_{2}$ & $-\gamma_{013}$ & $-\gamma_{123}$ & $\gamma_{0}$ & $\gamma_{0123}$ & $-\mathrm{I}$ & $-\gamma_{02}$ & $\gamma_{13}$ \\
\hline$\gamma_{0}$ & $\gamma_{0}$ & $\gamma_{123}$ & $\gamma_{013}$ & $\gamma_{2}$ & $\gamma_{13}$ & $\gamma_{02}$ & $\mathrm{I}$ & $\gamma_{0123}$ \\
\hline$\gamma_{123}$ & $\gamma_{123}$ & $-\gamma_{0}$ & $\gamma_{2}$ & $-\gamma_{013}$ & $-\gamma_{02}$ & $\gamma_{13}$ & $-\gamma_{0123}$ & $\mathrm{I}$ \\
\hline
\end{tabular}




\begin{tabular}{|c|c|c|c|c|c|c|c|c|}
\hline & 1 & $\mathrm{~W}$ & $E$ & $C$ & $\Pi$ & $\mathrm{K}$ & $S$ & $\bar{F}$ \\
\hline 1 & I & W & $E$ & $C$ & $\Pi$ & $K$ & $S$ & $\mathrm{~F}$ \\
\hline W & W & -1 & C & $-\Pi$ & $-K$ & $\Pi$ & $-\mathrm{F}$ & $S$ \\
\hline$E$ & $E$ & $C$ & -1 & $-W$ & $-\mathrm{S}$ & $-F$ & $\Pi$ & $\mathrm{K}$ \\
\hline C & C & $-E$ & $-W$ & I & $F$ & $-S$ & $-K$ & $\Pi$ \\
\hline$\Pi$ & $\Pi$ & K & $-S$ & $-\mathrm{F}$ & -1 & $-W$ & $E$ & C \\
\hline K & $\mathrm{K}$ & $-\Pi$ & $-F$ & $S$ & W & -1 & $-C$ & $E$ \\
\hline$S$ & $\mathrm{~S}$ & $\mathrm{~F}$ & $\Pi$ & K & E & C & I & W \\
\hline $\mathrm{F}$ & $\mathrm{F}$ & $-S$ & $\mathrm{~K}$ & $-\Pi$ & $-C$ & $E$ & $-W$ & I \\
\hline
\end{tabular}

As follows from this table, the group $\operatorname{Ext}\left(\mathbb{C}_{4}\right)$ is non-Abelian. Ext $\mathbb{C}_{+}\left(\mathbb{C}_{4}\right)$ contains Abelian group of spacetime reflections Aut $-\left(C \ell_{1,3}\right) \simeq \mathbb{Z}_{4}$ as a subgroup. It is easy to see that the group (911) is a group of the form Ext ${ }_{+}^{2}$ with order structure $(3,4)$. More precisely, the group (91) is a finite group $\stackrel{*}{\mathbb{Z}}_{4} \otimes \mathbb{Z}_{2}$ with the signature $(-,-,+,-,-,+,+)$.

Coming back to example 1 we see that the groups (69) and (191) are isomorphic:

$$
\{1, P, T, P T, C, C P, C T, C P T\} \simeq\{\mathrm{I}, \mathrm{W}, \mathrm{E}, \mathrm{C}, \Pi, \mathrm{K}, \mathrm{S}, \mathrm{F}\} \simeq \mathbb{Z}_{4}^{*} \otimes \mathbb{Z}_{2} .
$$

Moreover, the subgroups of spacetime reflections of these groups are also isomorphic:

$$
\{1, P, T, P T\} \simeq\{\mathrm{I}, \mathrm{W}, \mathrm{E}, \mathrm{C}\} \simeq \mathbb{Z}_{4} .
$$

Thus, we come to the following result: the finite group (69), derived from the analysis of invariance properties of the Dirac equation with respect to discrete transformations $C, P$ and $T$, is isomorphic to an extended automorphism group of the Dirac algebra $\mathbb{C}_{4}$. This result allows us to study discrete symmetries and their group structure for physical fields of any spin (without handling to analysis of relativistic wave equations).

\section{Clifford-Lipschitz groups}

The Lipschitz group $\boldsymbol{\Gamma}_{p, q}$, also called the Clifford group, introduced by Lipschitz in 1886 [54], may be defined as the subgroup of invertible elements $s$ of 
the algebra $C_{p, q}$ :

$$
\boldsymbol{\Gamma}_{p, q}=\left\{s \in C \ell_{p, q}^{+} \cup C \ell_{p, q}^{-} \mid \forall \mathbf{x} \in \mathbb{R}^{p, q}, s \mathbf{x} s^{-1} \in \mathbb{R}^{p, q}\right\} .
$$

The set $\boldsymbol{\Gamma}_{p, q}^{+}=\boldsymbol{\Gamma}_{p, q} \cap C \ell_{p, q}^{+}$is called special Lipschitz group [21].

Let $N: C \ell_{p, q} \rightarrow C \ell_{p, q}, N(\mathbf{x})=\mathbf{x} \widetilde{\mathbf{x}}$. If $\mathbf{x} \in \mathbb{R}^{p, q}$, then $N(\mathbf{x})=\mathbf{x}(-\mathbf{x})=$ $-\mathbf{x}^{2}=-Q(\mathbf{x})$. Further, the group $\boldsymbol{\Gamma}_{p, q}$ has a subgroup

$$
\operatorname{Pin}(p, q)=\left\{s \in \boldsymbol{\Gamma}_{p, q} \mid N(s)= \pm 1\right\}
$$

Analogously, a spinor group $\operatorname{Spin}(p, q)$ is defined by the set

$$
\operatorname{Spin}(p, q)=\left\{s \in \Gamma_{p, q}^{+} \mid N(s)= \pm 1\right\} .
$$

It is obvious that $\operatorname{Spin}(p, q)=\mathbf{P i n}(p, q) \cap C_{p, q}^{+}$. The group $\operatorname{Spin}(p, q)$ contains a subgroup

$$
\operatorname{Spin}_{+}(p, q)=\{s \in \operatorname{Spin}(p, q) \mid N(s)=1\} .
$$

The groups $O(p, q), S O(p, q)$ and $S O_{+}(p, q)$ are isomorphic, respectively, to the following quotient groups

$$
\begin{aligned}
O(p, q) & \simeq \operatorname{Pin}(p, q) / \mathbb{Z}_{2}, \\
S O(p, q) & \simeq \operatorname{Spin}(p, q) / \mathbb{Z}_{2}, \\
S O_{+}(p, q) & \simeq \operatorname{Spin}_{+}(p, q) / \mathbb{Z}_{2},
\end{aligned}
$$

where the kernel $\mathbb{Z}_{2}=\{1,-1\}$. Thus, the groups $\operatorname{Pin}(p, q), \operatorname{Spin}(p, q)$ and $\operatorname{Spin}_{+}(p, q)$ are the universal coverings of the groups $O(p, q), S O(p, q)$ and $\mathrm{SO}_{+}(p, q)$, respectively.

Further, since $C \ell_{p, q}^{+} \simeq C \ell_{q, p}^{+}$, then

$$
\operatorname{Spin}(p, q) \simeq \operatorname{Spin}(q, p)
$$

In contrast with this, the groups $\operatorname{Pin}(p, q)$ and $\operatorname{Pin}(q, p)$ are non-isomorphic. Let us denote $\operatorname{Spin}(n)=\operatorname{Spin}(n, 0) \simeq \operatorname{Spin}(0, n)$.

Theorem 7 ([26]). The spinor groups

$$
\operatorname{Spin}(2), \quad \operatorname{Spin}(3), \quad \operatorname{Spin}(4), \quad \operatorname{Spin}(5), \quad \operatorname{Spin}(6)
$$

are isomorphic to the unitary groups

$$
U(1), \quad S p(1) \sim S U(2), \quad S U(2) \times S U(2), \quad S p(2), \quad S U(4) .
$$


In case of the types $p-q \equiv 1,5(\bmod 8)$ the algebra $C \ell_{p, q}$ is isomorphic to a direct sum of two mutually annihilating simple ideals $\frac{1}{2}(1 \pm \omega) C \ell_{p, q}: C \ell_{p, q} \simeq$ $\frac{1}{2}(1+\omega) C \ell_{p, q} \oplus \frac{1}{2}(1-\omega) C \ell_{p, q}$, where $\omega=\mathbf{e}_{12 \ldots p+q}, p-q \equiv 1,5(\bmod 8)$. At this point, the each ideal is isomorpic to $C_{p, q-1}$ or $C_{q, p-1}$. Therefore, for the Clifford-Lipschitz groups we have the following isomorphisms

$$
\begin{aligned}
\operatorname{Pin}(p, q) & \simeq \operatorname{Pin}(p, q-1) \bigcup \operatorname{Pin}(p, q-1) \\
& \simeq \operatorname{Pin}(q, p-1) \bigcup \operatorname{Pin}(q, p-1)
\end{aligned}
$$

Or, since $C \ell_{p, q-1} \simeq C \ell_{p, q}^{+} \subset C \ell_{p, q}$, then according to (94)

$$
\operatorname{Pin}(p, q) \simeq \operatorname{Spin}(p, q) \bigcup \operatorname{Spin}(p, q)
$$

if $p-q \equiv 1,5(\bmod 8)$.

Further, in the case of $p-q \equiv 3,7(\bmod 8)$ the $C \ell_{p, q}$ is isomorphic to a complex algebra $\mathbb{C}_{p+q-1}$. Therefore, for the Pin groups we obtain

$$
\begin{aligned}
\operatorname{Pin}(p, q) & \simeq \operatorname{Pin}(p, q-1) \bigcup \mathbf{e}_{12 \ldots p+q} \operatorname{Pin}(p, q-1) \\
& \simeq \operatorname{Pin}(q, p-1) \bigcup \mathbf{e}_{12 \ldots p+q} \operatorname{Pin}(q, p-1)
\end{aligned}
$$

if $p-q \equiv 1,5(\bmod 8)$ and correspondingly

$$
\operatorname{Pin}(p, q) \simeq \operatorname{Spin}(p, q) \bigcup \mathbf{e}_{12 \ldots p+q} \operatorname{Spin}(p, q)
$$

In case of $p-q \equiv 3,7(\bmod 8)$ we have isomorphisms which are analoguos to (96)-(97), since $\omega C \ell_{p, q} \sim C \ell_{p, q}$. Generalizing we obtain the following

Theorem 8. Let $\operatorname{Pin}(p, q)$ and $\operatorname{Spin}(p, q)$ be the Clifford-Lipschitz groups of the invertible elements of the algebras $C_{p, q}$ with odd dimensionality, $p-q \equiv$ 1, 3, 5, $7(\bmod 8)$. Then

$$
\begin{aligned}
\operatorname{Pin}(p, q) & \simeq \operatorname{Pin}(p, q-1) \bigcup \omega \operatorname{Pin}(p, q-1) \\
& \simeq \operatorname{Pin}(q, p-1) \bigcup \omega \operatorname{Pin}(q, p-1)
\end{aligned}
$$

and

$$
\operatorname{Pin}(p, q) \simeq \operatorname{Spin}(p, q) \bigcup \omega \operatorname{Spin}(p, q)
$$

where $\omega=\mathbf{e}_{12 \ldots p+q}$ is a volume element of $C \ell_{p, q}$.

In case of low dimensionalities from Theorem 7 and Theorem 8 it immediately follows 
Theorem 9. For $p+q \leq 5$ and $p-q \equiv 3,5(\bmod 8)$,

$$
\begin{aligned}
& \operatorname{Pin}(3,0) \simeq S U(2) \cup i S U(2), \\
& \operatorname{Pin}(0,3) \simeq S U(2) \cup e S U(2), \\
& \operatorname{Pin}(5,0) \simeq S p(2) \cup e S p(2), \\
& \operatorname{Pin}(0,5) \simeq S p(2) \cup i S p(2) .
\end{aligned}
$$

Proof. Indeed, in accordance with Theorem $8 \operatorname{Pin}(3,0) \simeq \operatorname{Spin}(3) \cup$ $\mathbf{e}_{123} \operatorname{Spin}(3)$. Further, from Theorem 7 we have $\operatorname{Spin}(3) \simeq S U(2)$, and a square of the element $\omega=\mathbf{e}_{123}$ is equal to -1 , therefore, $\omega \sim i$. Thus, $\operatorname{Pin}(3,0) \simeq S U(2) \cup i S U(2)$. For the group $\operatorname{Pin}(0,3)$ a square of $\omega$ is equal to +1 , therefore, $\operatorname{Pin}(0,3) \simeq S U(2) \cup e S U(2)$, $e$ is a double unit. As expected, $\operatorname{Pin}(3,0) \not 千 \operatorname{Pin}(0,3)$. The isomorphisms for the groups $\operatorname{Pin}(5,0)$ and $\operatorname{Pin}(0,5)$ are analogously proved.

In turn, over the field $\mathbb{F}=\mathbb{C}$ there exists a complex Clifford-Lipschitz group

$$
\Gamma_{n}=\left\{s \in \mathbb{C}_{n}^{+} \cup \mathbb{C}_{n}^{-} \mid \forall \mathbf{x} \in \mathbb{C}^{n}, s \mathbf{x} s^{-1} \in \mathbb{C}^{n}\right\} .
$$

The group $\boldsymbol{\Gamma}_{n}$ has a subgroup

$$
\operatorname{Pin}(n, \mathbb{C})=\left\{s \in \boldsymbol{\Gamma}_{n} \mid N(s)= \pm 1\right\} .
$$

$\operatorname{Pin}(n, \mathbb{C})$ is an universal covering of the complex orthogonal group $O(n, \mathbb{C})$. When $n \equiv 1(\bmod 2)$ we have

$$
\operatorname{Pin}(n, \mathbb{C}) \simeq \operatorname{Pin}(n-1, \mathbb{C}) \bigcup \mathbf{e}_{12 \cdots n} \operatorname{Pin}(n-1, \mathbb{C}) .
$$

\section{$5.1 \quad P T$-structures}

On the other hand, there exists a more detailed version of the Pin-group (92) proposed by Dąbrowski in 1988 [30]. In general, there are eight double coverings of the orthogonal group $O(p, q)$ [30, 11]:

$$
\rho^{a, b, c}: \operatorname{Pin}^{a, b, c}(p, q) \longrightarrow O(p, q),
$$

where $a, b, c \in\{+,-\}$. As known, the group $O(p, q)$ consists of four connected components: identity connected component $O_{0}(p, q)$, and three components corresponding to space inversion $P$, time reversal $T$, and the combination of these two $P T$, i.e., $O(p, q)=\left(O_{0}(p, q)\right) \cup P\left(Q_{0}(p, q)\right) \cup$ $T\left(O_{0}(p, q)\right) \cup P T\left(O_{0}(p, q)\right)$. Further, since the four-element group (reflection 
Table 4. PT-structures.

\begin{tabular}{|c|l|l|}
\hline$a b c$ & $C^{a, b, c}$ & Remark \\
\hline+++ & $\mathbb{Z}_{2} \otimes \mathbb{Z}_{2} \otimes \mathbb{Z}_{2}$ & $P T=T P$ \\
+-- & $\mathbb{Z}_{2} \otimes \mathbb{Z}_{4}$ & $P T=T P$ \\
-+- & $\mathbb{Z}_{2} \otimes \mathbb{Z}_{4}$ & $P T=T P$ \\
--+ & $\mathbb{Z}_{2} \otimes \mathbb{Z}_{4}$ & $P T=T P$ \\
\hline--- & $Q_{4}$ & $P T=-T P$ \\
-++ & $D_{4}$ & $P T=-T P$ \\
+-+ & $D_{4}$ & $P T=-T P$ \\
++- & $D_{4}$ & $P T=-T P$ \\
\hline
\end{tabular}

group) $\{1, P, T, P T\}$ is isomorphic to the finite group $\mathbb{Z}_{2} \otimes \mathbb{Z}_{2}$ (Gauss-Klein viergruppe [70, 72]), then $O(p, q)$ may be represented by a semidirect product $O(p, q) \simeq O_{0}(p, q) \odot\left(\mathbb{Z}_{2} \otimes \mathbb{Z}_{2}\right)$. The signs of $a, b, c$ correspond to the signs of the squares of the elements in $\mathbf{P i n}^{a, b, c}(p, q)$ which cover space inversion $P$, time reversal $T$ and a combination of these two $P T\left(a=-P^{2}, b=T^{2}, c=-(P T)^{2}\right.$ in Dạbrowski's notation [30] and $a=P^{2}, b=T^{2}, c=(P T)^{2}$ in Chamblin's notation [18] which we will use below). An explicit form of the group $\mathbf{P i n}^{a, b, c}(p, q)$ is given by the following semidirect product

$$
\operatorname{Pin}^{a, b, c}(p, q) \simeq \frac{\left(\operatorname{Spin}_{+}(p, q) \odot C^{a, b, c}\right)}{\mathbb{Z}_{2}},
$$

where $C^{a, b, c}$ are the four double coverings of $\mathbb{Z}_{2} \otimes \mathbb{Z}_{2}$. All the eight universal coverings of the orthogonal group $O(p, q)$ are given in the Table 4 . Here $\mathbb{Z}_{4}, Q_{4}$, and $D_{4}$ are complex, quaternion, and dihedral groups, respectively. According to 30] the group $\operatorname{Pin}^{a, b, c}(p, q)$ satisfying the condition $P T=-T P$ is called Cliffordian, and respectively non-Cliffordian when $P T=T P$. Taking into account the Proposition 1 and Theorems 4 and 5. we come to the following

Theorem $10([78])$. Let $\mathbf{P i n}^{a, b, c}(p, q)$ be an universal covering of the complex orthogonal group $O(n, \mathbb{C})$ of the space $\mathbb{C}^{n}$ associated with the complex algebra $\mathbb{C}_{n}$. Squares of the symbols $a, b, c \in\{-,+\}$ are correspond to squares of the elements of the finite group Aut $=\{\mathrm{I}, \mathrm{W}, \mathrm{E}, \mathrm{C}\}: a=\mathrm{W}^{2}, b=\mathrm{E}^{2}, c=\mathrm{C}^{2}$, where $\mathrm{W}, \mathrm{E}$ and $\mathrm{C}$ are correspondingly the matrices of the fundamental automorphisms $\mathcal{A} \rightarrow \mathcal{A}^{\star}, \mathcal{A} \rightarrow \widetilde{\mathcal{A}}$ and $\mathcal{A} \rightarrow \widetilde{\mathcal{A}^{\star}}$ of $\mathbb{C}_{n}$. Then over the field $\mathbb{F}=\mathbb{C}$ 
for the algebra $\mathbb{C}_{n}$ there exist two non-isomorphic universal coverings of the group $O(n, \mathbb{C})$ :

1) Non-Cliffordian groups

$$
\operatorname{Pin}^{+,+,+}(n, \mathbb{C}) \simeq \frac{\left(\operatorname{Spin}_{+}(n, \mathbb{C}) \odot \mathbb{Z}_{2} \otimes \mathbb{Z}_{2} \otimes \mathbb{Z}_{2}\right)}{\mathbb{Z}_{2}}
$$

if $n \equiv 0(\bmod 4)$ and

$$
\operatorname{Pin}^{+,+,+}(n, \mathbb{C}) \simeq \operatorname{Pin}^{+,+,+}(n-1, \mathbb{C}) \bigcup \mathbf{e}_{12 \ldots n} \operatorname{Pin}^{+,+,+}(n-1, \mathbb{C}),
$$

if $n \equiv 1(\bmod 4)$.

2) Cliffordian groups

$$
\operatorname{Pin}^{-,-,-}(n, \mathbb{C}) \simeq \frac{\left(\operatorname{Spin}_{+}(n, \mathbb{C}) \odot Q_{4}\right)}{\mathbb{Z}_{2}}
$$

if $n \equiv 2(\bmod 4)$ and

$$
\operatorname{Pin}^{-,-,-}(n, \mathbb{C}) \simeq \operatorname{Pin}^{-,-,-}(n-1, \mathbb{C}) \bigcup \mathbf{e}_{12 \ldots n} \operatorname{Pin}^{-,-,-}(n-1, \mathbb{C}),
$$

if $n \equiv 3(\bmod 4)$.

Theorem 11 (79]). Let $\mathbf{P i n}^{a, b, c}(p, q)$ be an universal covering of the orthogonal group $O(p, q)$ of the real space $\mathbb{R}^{p, q}$ associated with the algebra $C_{p, q}$. The squares of symbols $a, b, c \in\{-,+\}$ correspond to the squares of the elements of a finite group Aut $\left(C_{p, q}\right)=\{\mathrm{I}, \mathrm{W}, \mathrm{E}, \mathrm{C}\}: a=\mathrm{W}^{2}, b=\mathrm{E}^{2}, c=\mathrm{C}^{2}$, where $\mathrm{W}, \mathrm{E}$ and $\mathrm{C}$ are the matrices of the fundamental automorphisms $\mathcal{A} \rightarrow \mathcal{A}^{\star}, \mathcal{A} \rightarrow \widetilde{\mathcal{A}}$ and $\mathcal{A} \rightarrow \widetilde{\mathcal{A}^{\star}}$ of the algebra $C_{p, q}$, respectively. Then over the field $\mathbb{F}=\mathbb{R}$ in dependence on a division ring structure of the algebra $C_{p, q}$, there exist eight universal coverings of the orthogonal group $O(p, q)$ :

1) A non-Cliffordian group

$$
\operatorname{Pin}^{+,+,+}(p, q) \simeq \frac{\left(\operatorname{Spin}_{0}(p, q) \odot \mathbb{Z}_{2} \otimes \mathbb{Z}_{2} \otimes \mathbb{Z}_{2}\right)}{\mathbb{Z}_{2}},
$$

exists if $\mathbb{K} \simeq \mathbb{R}$ and the numbers $p$ and $q$ form the type $p-q \equiv 0(\bmod 8)$ and $p, q \equiv 0(\bmod 4)$, and also if $p-q \equiv 4(\bmod 8)$ and $\mathbb{K} \simeq \mathbb{H}$. The algebras $C_{p, q}$ with the rings $\mathbb{K} \simeq \mathbb{R} \oplus \mathbb{R}, \mathbb{K} \simeq \mathbb{H} \oplus \mathbb{H}(p-q \equiv 1,5(\bmod 8))$ admit the group $\mathbf{P i n}^{+,+,+}(p, q)$ if in the direct sums there are addendums of the type $p-q \equiv 0(\bmod 8)$ or $p-q \equiv 4(\bmod 8)$. The types $p-q \equiv 3,7(\bmod 8)$, 
$\mathbb{K} \simeq \mathbb{C}$ admit a non-Cliffordian group $\mathbf{P i n}^{+,+,+}(p+q-1, \mathbb{C})$ if $p \equiv 0(\bmod 2)$ and $q \equiv 1$ (mod 2). Further, non-Cliffordian groups

$$
\operatorname{Pin}^{a, b, c}(p, q) \simeq \frac{\left(\operatorname{Spin}_{0}(p, q) \odot\left(\mathbb{Z}_{2} \otimes \mathbb{Z}_{4}\right)\right.}{\mathbb{Z}_{2}}
$$

with $(a, b, c)=(+,-,-)$ exist if $p-q \equiv 0(\bmod 8), p, q \equiv 2(\bmod 4)$ and $\mathbb{K} \simeq \mathbb{R}$, and also if $p-q \equiv 4(\bmod 8)$ and $\mathbb{K} \simeq \mathbb{H}$. Non-Cliffordian groups with the signatures $(a, b, c)=(-,+,-)$ and $(a, b, c)=(-,-,+)$ exist over the ring $\mathbb{K} \simeq \mathbb{R}(p-q \equiv 2(\bmod 8))$ if $p \equiv 2(\bmod 4), q \equiv 0(\bmod 4)$ and $p \equiv 0(\bmod 4), q \equiv 2(\bmod 4)$, respectively, and also these groups exist over the ring $\mathbb{K} \simeq \mathbb{H}$ if $p-q \equiv 6(\bmod 8)$. The algebras $C_{p, q}$ with the rings $\mathbb{K} \simeq \mathbb{R} \oplus \mathbb{R}, \mathbb{K} \simeq \mathbb{H} \oplus \mathbb{H}(p-q \equiv 1,5(\bmod 8))$ admit the group $\mathbf{P i n}^{+,-,-}(p, q)$ if in the direct sums there are addendums of the type $p-q \equiv 0(\bmod 8)$ or $p-q \equiv 4(\bmod 8)$, and also admit the groups $\mathbf{P} \mathbf{i n}^{-,+,-}(p, q)$ and $\mathbf{P i n}^{-,-,+}(p, q)$ if in the direct sums there are addendums of the type $p-q \equiv 2(\bmod 8)$ or $p-q \equiv 6(\bmod 8)$.

\section{2) A Cliffordian group}

$$
\operatorname{Pin}^{-,-,-}(p, q) \simeq \frac{\left(\operatorname{Spin}_{0}(p, q) \odot Q_{4}\right)}{\mathbb{Z}_{2}}
$$

exists if $\mathbb{K} \simeq \mathbb{R}(p-q \equiv 2(\bmod 8))$ and $p \equiv 3(\bmod 4), q \equiv 1(\bmod 4)$, and also if $p-q \equiv 6(\bmod 8)$ and $\mathbb{K} \simeq \mathbb{H}$. The algebras $C_{p, q}$ with the rings $\mathbb{K} \simeq \mathbb{R} \oplus \mathbb{R}, \mathbb{K} \simeq \mathbb{H} \oplus \mathbb{H}(p-q \equiv 1,5(\bmod 8))$ admit the group $\mathbf{P i n}^{-,-,-}(p, q)$ if in the direct sums there are addendums of the type $p-q \equiv 2(\bmod 8)$ or $p-q \equiv 6(\bmod 8)$. The types $p-q \equiv 3,7(\bmod 8), \mathbb{K} \simeq \mathbb{C}$ admit a Cliffordian group $\mathbf{P i n}^{-,-}-(p+q-1, \mathbb{C})$, if $p \equiv 1(\bmod 2)$ and $q \equiv 0(\bmod 2)$. Further, Cliffordian groups

$$
\operatorname{Pin}^{a, b, c}(p, q) \simeq \frac{\left(\operatorname{Spin}_{0}(p, q) \odot D_{4}\right)}{\mathbb{Z}_{2}},
$$

with $(a, b, c)=(-,+,+)$ exist if $\mathbb{K} \simeq \mathbb{R}(p-q \equiv 2(\bmod 8))$ and $p \equiv 1$ $(\bmod 4), q \equiv 3(\bmod 4)$, and also if $p-q \equiv 6(\bmod 8)$ and $\mathbb{K} \simeq \mathbb{H}$. Cliffordian groups with the signatures $(a, b, c)=(+,-,+)$ and $(a, b, c)=(+,+,-)$ exist over the ring $\mathbb{K} \simeq \mathbb{R}(p-q \equiv 0(\bmod 8))$ if $p, q \equiv 3(\bmod 4)$ and $p, q \equiv 1$ $(\bmod 4)$, respectively, and also these groups exist over the ring $\mathbb{K} \simeq \mathbb{H}$ if $p-q \equiv 4(\bmod 8)$. The algebras $C_{p, q}$ with the rings $\mathbb{K} \simeq \mathbb{R} \oplus \mathbb{R}, \mathbb{K} \simeq \mathbb{H} \oplus \mathbb{H}$ $(p-q \equiv 1,5(\bmod 8))$ admit the group $\mathbf{P i n}^{-,+,+}(p, q)$ if in the direct sums there are addendums of the type $p-q \equiv 2(\bmod 8)$ or $p-q \equiv 6(\bmod 8)$, and also admit the groups $\mathbf{P i n}{ }^{+,-+}(p, q)$ and $\mathbf{P i n}{ }^{+,+,-}(p, q)$ if in the direct sums there are addendums of the type $p-q \equiv 0(\bmod 8)$ or $p-q \equiv 4(\bmod 8)$. 
Table 5. $C P T$-structures.

\begin{tabular}{|l|l|l|}
\hline a bcdefg & $C^{a, b, c, d, e, f, g}$ & Type \\
\hline+++++++ & $\mathbb{Z}_{2} \otimes \mathbb{Z}_{2} \otimes \mathbb{Z}_{2} \otimes \mathbb{Z}_{2}$ & Abelian \\
three '+' and four '-' & $\mathbb{Z}_{4} \otimes \mathbb{Z}_{2} \otimes \mathbb{Z}_{2}$ & \\
\hline one '+' and six '-' & $Q_{4} \otimes \mathbb{Z}_{2}$ & Non-Abelian \\
five '+' and two '-', & $D_{4} \otimes \mathbb{Z}_{2}$ & \\
three '+' and four '-', & $\mathbb{Z}_{4} \otimes \mathbb{Z}_{2} \otimes \mathbb{Z}_{2}$ & \\
\hline
\end{tabular}

\section{$5.2 \quad C P T$-structures}

As it has been shown previously, there exist 64 different signatures $(a, b, c, d, e, f, g)$ for the extended automorphism group $\operatorname{Ext}\left(\mathbb{C}_{n}\right)$ of the complex Clifford algebra $\mathbb{C}_{n}$. At this point, the group of fundamental automorphisms, Aut $_{ \pm}\left(C \ell_{p, q}\right)$, which has 8 different signatures $(a, b, c)$, is defined as a subgroup of $\operatorname{Ext}\left(\mathbb{C}_{n}\right)$. As known, the Clifford-Lipschitz group $\operatorname{Pin}(n, \mathbb{C}$ ) (an universal covering of the complex orthogonal group $O(n, \mathbb{C}))$ is completely constructed within the algebra $\mathbb{C}_{n}$ (see definition (98)). If we take into account spacetime reflections, then according to [74, 75, 30] there exist 8 types of universal covering (PT-structures) described by the group $\mathbf{P i n}^{a, b, c}(n, \mathbb{C})$. As it shown in 78, 79], the group $\operatorname{Pin}^{a, b, c}(n, \mathbb{C})$ (correspondingly $\operatorname{Pin}^{a, b, c}(p, q)$ over the field $\mathbb{F}=\mathbb{R}$ ) is completely defined within the algebra $\mathbb{C}_{n}$ (correspondingly $C \ell_{p, q}$ ) by means of identification of the reflection subgroup $\{1, P, T, P T\}$ with the automorphism group $\{\mathrm{Id}, \star, \sim, \widetilde{\star}\}$ of $\mathbb{C}_{n}$ (correspondingly $C \ell_{p, q}$ ). In turn, the pseudoautomorphism $\mathcal{A} \rightarrow \overline{\mathcal{A}}$ of $\mathbb{C}_{n}$ and the extended automorphism group $\{\mathrm{Id}, \star, \sim, \widetilde{\star},-\bar{\star}, \bar{\sim}, \overline{\widetilde{\star}}\} \simeq \mathbb{Z}_{2} \otimes \mathbb{Z}_{2} \otimes \mathbb{Z}_{2}$ allows us to give a further generalization of the Clifford-Lipschitz group (98) (correspondingly (92)). We claim that there exist 64 types of the universal covering (CPT-structures) for the complex orthogonal group $O(n, \mathbb{C})$ :

$$
\operatorname{Pin}^{a, b, c, d, e, f, g}(n, \mathbb{C}) \simeq \frac{\left(\operatorname{Spin}_{+}(n, \mathbb{C}) \odot C^{a, b, c, d, e, f, g}\right)}{\mathbb{Z}_{2}},
$$

where $C^{a, b, c, d, e, f, g}$ are five double coverings of the group $\mathbb{Z}_{2} \otimes \mathbb{Z}_{2} \otimes \mathbb{Z}_{2}$. All the posiible double coverings $C^{a, b, c, d, e, f, g}$ are given in the Table 5. The group (101) with non-Abelian $C^{a, b, c, d, e, f, g}$ we will call Cliffordian and respectively non-Cliffordian when $C^{a, b, c, d, e, f, g}$ is Abelian.

Analogously, over the field $\mathbb{F}=\mathbb{R}$ there exist 64 universal coverings of the 
real orthogonal group $O(p, q)$ :

$$
\rho^{a, b, c, d, e, f, g}: \operatorname{Pin}^{a, b, c, d, e, f, g} \longrightarrow O(p, q),
$$

where

$$
\operatorname{Pin}^{a, b, c, d, e, f, g}(p, q) \simeq \frac{\left(\operatorname{Spin}_{+}(p, q) \odot C^{a, b, c, d, e, f, g}\right)}{\mathbb{Z}_{2}} .
$$

It is easy to see that in case of the algebra $C \ell_{p, q}$ (or subalgebra $C \ell_{p, q} \subset \mathbb{C}_{n}$ ) with the real division $\operatorname{ring} \mathbb{K} \simeq \mathbb{R}, p-q \equiv 0,2(\bmod 8), C P T$-structures, defined by the groups (101) and (102), are reduced to the eight Shirokov-Dạbrowski PT-structures.

Further, using the well-known isomorphism (99) we obtain for the group $O(n, \mathbb{C})$ with odd dimensionality the following universal covering:

$$
\operatorname{Pin}^{a, b, c, d, e, f, g}(n, \mathbb{C}) \simeq \operatorname{Pin}^{a, b, c, d, e, f, g}(n-1, \mathbb{C}) \bigcup \mathbf{e}_{12 \cdots n} \operatorname{Pin}^{a, b, c, d, e, f, g}(n-1, \mathbb{C}) .
$$

Correspondingly, in virtue of $C_{p, q} \simeq \mathbb{C}_{n-1}(p-q \equiv 3,7(\bmod 8), n=p+q)$ and (95) for the group $O(p, q)$ with odd dimensionality we have

$$
\operatorname{Pin}^{a, b, c, d, e, f, g}(p, q) \simeq \operatorname{Pin}^{a, b, c, d, e, f, g}(n-1, \mathbb{C})
$$

for $p-q \equiv 3,7(\bmod 8)$ and

$$
\begin{aligned}
& \operatorname{Pin}^{a, b, c, d, e, f, g}(p, q) \simeq \operatorname{Pin}^{a, b, c, d, e, f, g}(p, q-1) \bigcup \mathbf{e}_{12 \cdots n} \operatorname{Pin}^{a, b, c, d, e, f, g}(p, q-1) \\
& \operatorname{Pin}^{a, b, c, d, e, f, g}(p, q) \simeq \operatorname{Pin}^{a, b, c, d, e, f, g}(q, p-1) \bigcup \mathbf{e}_{12 \cdots n} \operatorname{Pin}^{a, b, c, d, e, f, g}(q, p-1)
\end{aligned}
$$

for the types $p-q \equiv 1,5(\bmod 8)$. Hence it immediately follows

Theorem 12. Let $\mathbf{P i n}^{a, b, c, d, e, f, g}(n, \mathbb{C})$ be an universal covering of the complex orthogonal group $O(n, \mathbb{C})$ of the space $\mathbb{C}^{n}$ associated with the complex algebra $\mathbb{C}_{n}$. Squares of the symbols $a, b, c, d, e, f, g \in\{-,+\}$ correspond to squares of the elements of the finite group $\mathrm{Ext}=\{\mathrm{I}, \mathrm{W}, \mathrm{E}, \mathrm{C}, \Pi, \mathrm{K}, \mathrm{S}, \mathrm{F}\}: a=\mathrm{W}^{2}, b=\mathrm{E}^{2}$, $c=\mathrm{C}^{2}, d=\Pi^{2}, e=\mathrm{K}^{2}, f=\mathrm{S}^{2}, g=\mathrm{F}^{2}$, where $\mathrm{W}, \mathrm{E}, \mathrm{C}, \Pi, \mathrm{K}, \mathrm{S}, \mathrm{F}$ are spinor representations of the automorphisms $\mathcal{A} \rightarrow \mathcal{A}^{\star}, \mathcal{A} \rightarrow \widetilde{\mathcal{A}}, \mathcal{A} \rightarrow \widetilde{\mathcal{A}^{\star}}, \mathcal{A} \rightarrow \overline{\mathcal{A}}$, $\mathcal{A} \rightarrow \overline{\mathcal{A}^{\star}}, \mathcal{A} \rightarrow \overline{\widetilde{\mathcal{A}}}, \mathcal{A} \rightarrow \overline{\widetilde{\mathcal{A}^{\star}}}$. Then over the field $\mathbb{F}=\mathbb{C}$ in dependence on a division ring structure $\mathbb{K}=f \ell_{p, q} f$ of the real subalgebras $C_{p, q} \subset \mathbb{C}_{n}$, there exist the following universal coverings (CPT-structures) of the group $O(n, \mathbb{C})$ :

1) $\mathbb{K} \simeq \mathbb{R}, p-q \equiv 0,2(\bmod 8)$.

In this case CPT-structures are reduced to the eight Shirokov-Dabrowski PTstructures

$$
\operatorname{Pin}^{a, b, c}(n, \mathbb{C}) \simeq \frac{\left(\operatorname{Spin}_{+}(n, \mathbb{C}) \odot C^{a, b, c}\right)}{\mathbb{Z}_{2}},
$$


where $C^{a, b, c}$ are double coverings of the group $\{1, P, T, P T\} \simeq\{\mathrm{I}, \mathrm{W}, \mathrm{E}, \mathrm{C}\} \simeq$ $\mathbb{Z}_{2} \otimes \mathbb{Z}_{2}$.

2) $\mathbb{K} \simeq \mathbb{H}, p-q \equiv 4,6(\bmod 8)$.

In this case we have 64 universal coverings:

a) Non-Cliffordian group

$$
\operatorname{Pin}^{+,+,+,+,++,+}(n, \mathbb{C}) \simeq \frac{\left(\operatorname{Spin}_{+}(n, \mathbb{C}) \odot \mathbb{Z}_{2} \otimes \mathbb{Z}_{2} \otimes \mathbb{Z}_{2} \otimes \mathbb{Z}_{2}\right)}{\mathbb{Z}_{2}}
$$

exists if the subalgebra $C_{p, q}$ admits the type $p-q \equiv 4(\bmod 8)$. NonCliffordian groups

$$
\operatorname{Pin}^{a, b, c, d, e, f, g}(n, \mathbb{C}) \simeq \frac{\left(\operatorname{Spin}_{+}(n, \mathbb{C}) \odot \mathbb{Z}_{4} \otimes \mathbb{Z}_{2} \otimes \mathbb{Z}_{2}\right)}{\mathbb{Z}_{2}}
$$

exist with the signature $(+,+,+,-,-,-,-)$ when $C_{p, q}$ has the type $p-q \equiv 4$ $(\bmod 8)$, and also these groups with the signatures $(a, b, c, d, e, f, g)$ exist when $p-q \equiv 6(\bmod 8)$, where among the symbols $a, b, c$ there are two minuses and one plus, and among $d, e, f, g$ - two pluses and two minuses.

b) Cliffordian groups

$$
\operatorname{Pin}^{a, b, c, d, e, f, g}(n, \mathbb{C}) \simeq \frac{\left(\operatorname{Spin}_{+}(n, \mathbb{C}) \odot Q_{4} \otimes \mathbb{Z}_{2}\right)}{\mathbb{Z}_{2}}
$$

exist with the signature $(+,-,-,-,-,-,-)$ when $p-q \equiv 4(\bmod 8)$ and with the signatures $(-,+,-,-,-,-,-),(-,-,+,-,-,-,-)$ when $p-q \equiv 6$ $(\bmod 8)$. And also these groups exist with the signature $(-,-,-, d, e, f, g)$ if $p-q \equiv 6(\bmod 8)$, where among the symbols $d, e, f, g$ there are one plus and three minuses. Cliffordian groups

$$
\operatorname{Pin}^{a, b, c, d, e, f, g}(n, \mathbb{C}) \simeq \frac{\left(\operatorname{Spin}_{+}(n, \mathbb{C}) \odot D_{4} \otimes \mathbb{Z}_{2}\right)}{\mathbb{Z}_{2}}
$$

exist with the signatures $(+,-,-,+,+,+,+)$ and $(+,+,+, d, e, f, g)$ when $C_{p, q}$ has the type $p-q \equiv 4(\bmod 8)$ and among $d, e, f, g$ there are two minuses and two pluses, and also these groups exist with $(+,-,+, d, e, f, g)$ and $(+,+,-, d, e, f, g)$, where among $d, e, f, g$ there are three pluses and one minus. Cliffordian groups of this type exist also with the signatures $(a, b, c,+,+,+,+)$ and $(-,+,+, d, e, f, g)$ when $p-q \equiv 6(\bmod 8)$, where among $a, b, c$ there are two minuses and one plus, and among $d, e, f, g$ there are three pluses and one minus. Cliffordian groups 


$$
\operatorname{Pin}^{a, b, c, d, e, f, g}(n, \mathbb{C}) \simeq \frac{\left(\operatorname{Spin}_{+}(n, \mathbb{C}) \odot \mathbb{Z}_{4}^{*} \otimes \mathbb{Z}_{2} \otimes \mathbb{Z}_{2}\right)}{\mathbb{Z}_{2}}
$$

exist with the signatures $(+,-,-, d, e, f, g)$ when $p-q \equiv 4(\bmod 8)$ and among the symbols $d, e, f, g$ there are two pluses and two minuses, and also these groups exist with $(+,-,+, d, e, f, g)$ and $(+,+,-, d, e, f, g)$, where $p-q \equiv 4$ $(\bmod 8)$ and among $d, e, f, g$ there are one plus and three minuses. Cliffordian groups of this type exist also with $(-,+,-, d, e, f, g)$ and $(-,-,+, d, e, f, g)$ if $p-q \equiv 6(\bmod 8)$, where among $d, e, f, g$ there are two pluses and two minuses. And also these groups exist with $(-,-,-, d, e, f, g)$ if $p-q \equiv 6$ ( $\bmod 8)$, where among $d, e, f, g$ there are three pluses and one minus.

\section{Quotient representations of the Clifford-Lipschitz groups}

In accordance with (11) and (12), the map $\gamma$ gives the left-regular spinor representation $\mathfrak{R}$ of $C \ell(Q)$ in $\mathbb{S}$ and $\mathbb{S} \oplus \mathbb{S}$, respectively. The representation $\mathfrak{R}$ is faithful if its kernel is zero, that is, $\mathfrak{R}(a) x=0, \forall x \in \mathbb{S} \Rightarrow a=0$. If the representation $\mathfrak{R}$ has only two invariant subspaces $\mathbb{S}$ and $\{0\}$, then $\mathfrak{R}$ is said to be simple or irreducible. On the contrary case, $\mathfrak{R}$ is said to be semi-simple, that is, it is a direct sum of simple modules, and in this case $\mathbb{S}$ is a direct sum of subspaces which are globally invariant under $\mathfrak{R}(a)$, $\forall a \in C \ell(Q)$. In virtue of the definition (92), the representation $\mathfrak{R}$ of $C_{p, q}$ induces a representation of $\operatorname{Pin}(p, q)$ which we will denote by the same symbol $\mathfrak{R}$, and also induces a representation of the $\operatorname{group} \operatorname{Spin}(p, q)$ which we will denote by $\mathfrak{R}^{+}$. Analogously, in virtue of (98), the representation $\mathfrak{C}$ of the complex algebra $\mathbb{C}_{n}$ induces a representation of $\operatorname{Pin}(n, \mathbb{C})$ which we will denote also by the same symbol $\mathfrak{C}$.

Further, over the field $\mathbb{F}=\mathbb{R}$ at $p+q \equiv 0(\bmod 2)$ there are four types of real algebras $C \ell_{p, q}$ : two types $p-q \equiv 0,2(\bmod 8)$ with a real division ring $\mathbb{K} \simeq \mathbb{R}$ and two types $p-q \equiv 4,6(\bmod 8)$ with a quaternionic division ring $\mathbb{K} \simeq \mathbb{H}$. Thus, in this case the representation $\mathfrak{R}$ of $\operatorname{Pin}(p, q)$ is divided into the following four classes:

$$
\begin{array}{lll}
\mathfrak{R}_{m}^{0} \leftrightarrow C \ell_{p, q}, p-q \equiv 0 & (\bmod 8), \mathbb{K} \simeq \mathbb{R} \\
\mathfrak{R}_{m}^{2} \leftrightarrow C \ell_{p, q}, p-q \equiv 2 & (\bmod 8), \mathbb{K} \simeq \mathbb{R}, \\
\mathfrak{H}_{m}^{4} \leftrightarrow C \ell_{p, q}, p-q \equiv 4 & (\bmod 8), \mathbb{K} \simeq \mathbb{H}, \\
\mathfrak{H}_{m}^{6} \leftrightarrow C \ell_{p, q}, p-q \equiv 6 & (\bmod 8), \mathbb{K} \simeq \mathbb{H},
\end{array}
$$


Table 6. Real representations of $\operatorname{Pin}(p, q)$.

\begin{tabular}{|c|c|c|c|c|c|c|c|c|c|}
\hline & $\mathrm{p}$ & 1 & 2 & 3 & 4 & 5 & 6 & 7 & $\ldots$ \\
\hline q & & & & & & & & & \\
\hline 0 & $\mathfrak{R}_{0}^{0}$ & ${ }^{2} \mathfrak{R}_{0}^{0}$ & $\mathfrak{R}_{1}^{2}$ & $\mathfrak{C}_{1}^{3}$ & $\mathfrak{H}_{1}^{4}$ & ${ }^{2} \mathfrak{H}_{1}^{4}$ & $\mathfrak{H}_{2}^{6}$ & $\mathfrak{C}_{4}^{7}$ & $\ldots$ \\
\hline 1 & $\mathfrak{C}_{0}^{7}$ & $\mathfrak{R}_{1}^{0}$ & ${ }^{2} \mathfrak{R}_{1}^{0}$ & $\mathfrak{R}_{2}^{2}$ & $\mathfrak{C}_{2}^{3}$ & $\mathfrak{H}_{2}^{4}$ & ${ }^{2} \mathfrak{H}_{2}^{4}$ & $\mathfrak{H}_{4}^{6}$ & $\ldots$ \\
\hline 2 & $\mathfrak{H}_{0}^{6}$ & $\mathfrak{C}_{1}^{7}$ & $\mathfrak{R}_{2}^{0}$ & ${ }^{2} \mathfrak{R}_{2}^{0}$ & $\mathfrak{R}_{4}^{2}$ & $\mathfrak{C}_{4}^{3}$ & $\mathfrak{H}_{4}^{4}$ & ${ }^{2} \mathfrak{H}_{4}^{4}$ & $\cdots$ \\
\hline 3 & ${ }^{2} \mathfrak{H}_{0}^{4}$ & $\mathfrak{H}_{1}^{6}$ & $\mathfrak{C}_{2}^{7}$ & $\mathfrak{R}_{4}^{0}$ & ${ }^{2} \mathfrak{R}_{4}^{0}$ & $\mathfrak{R}_{8}^{2}$ & $\mathfrak{C}_{8}^{3}$ & $\mathfrak{H}_{8}^{4}$ & \\
\hline 4 & $\mathfrak{H}_{1}^{4}$ & ${ }^{2} \mathfrak{H}_{1}^{4}$ & $\mathfrak{H}_{2}^{6}$ & $\mathfrak{C}_{4}^{7}$ & $\mathfrak{R}_{8}^{0}$ & ${ }^{2} \mathfrak{R}_{8}^{0}$ & $\mathfrak{R}_{16}^{2}$ & $\mathfrak{C}_{16}^{3}$ & $\cdots$ \\
\hline 5 & $\mathfrak{C}_{2}^{3}$ & $\mathfrak{H}_{2}^{4}$ & ${ }^{2} \mathfrak{H}_{2}^{4}$ & $\mathfrak{H}_{4}^{6}$ & $\mathfrak{C}_{8}^{7}$ & $\mathfrak{R}_{16}^{0}$ & ${ }^{2} \mathfrak{R}_{16}^{0}$ & $\mathfrak{R}_{32}^{2}$ & $\ldots$ \\
\hline 6 & $\mathfrak{R}_{4}^{2}$ & $\mathfrak{C}_{4}^{3}$ & $\mathfrak{H}_{4}^{4}$ & ${ }^{2} \mathfrak{H}_{4}^{4}$ & $\mathfrak{H}_{8}^{6}$ & $\mathfrak{C}_{16}^{7}$ & $\mathfrak{R}_{32}^{0}$ & ${ }^{2} \mathfrak{R}_{32}^{0}$ & \\
\hline 7 & ${ }^{2} \mathfrak{R}_{4}^{0}$ & $\mathfrak{R}_{8}^{2}$ & $\mathfrak{C}_{8}^{3}$ & $\mathfrak{H}_{8}^{4}$ & ${ }^{2} \mathfrak{H}_{8}^{4}$ & $\mathfrak{H}_{16}^{6}$ & $\mathfrak{C}_{32}^{7}$ & $\mathfrak{R}_{64}^{0}$ & $\cdots$ \\
\hline$\vdots$ & $\vdots$ & $\vdots$ & $\vdots$ & $:$ & $\vdots$ & $\vdots$ & $\vdots$ & $\vdots$ & \\
\hline
\end{tabular}

here $m=\frac{p+q}{2}$. We will call the representations $\mathfrak{H}_{m}^{4}$ and $\mathfrak{H}_{m}^{6}$ as quaternionic representations of the group $\operatorname{Pin}(p, q)$. Using the correspondence (103), we can define the Periodic Table for the real representations of $\operatorname{Pin}(p, q)$ (see the Table 6). On the other hand, in terms of minimal left ideal the modulo 8 periodicity looks like

$$
\mathbb{S}_{n+8} \simeq \mathbb{S}_{n} \otimes \mathbb{S}_{16}
$$

In virtue of the mapping $\gamma_{8,0}: C_{8,0} \rightarrow \mathrm{M}_{2}(\mathbb{O})$ [57] (see also excellent review [5]) the latter relation can be written in the form

$$
\mathbb{S}_{n+8} \simeq \mathbb{S}_{n} \otimes \mathbb{O}^{2}
$$

where $\mathbb{O}$ is an octonion algebra. Since the algebra $C \ell_{8,0} \simeq C \ell_{0,8}$ admits an octonionic representation, then in virtue of the modulo 8 periodicity the octonionic representations can be defined for all high dimensions and, therefore, on the system of real representations of $\operatorname{Pin}(p, q)$ we have a relation

$$
\mathfrak{D}^{m+2} \simeq \mathfrak{D}^{m} \otimes \mathfrak{O},
$$

where $\mathfrak{O}$ is an octonionic representation of the group $\operatorname{Pin}(p, q)\left(\mathfrak{O} \sim \mathfrak{R}_{4}^{0}\right)$.

As known [65, 61, the odd-dimensional algebras $C_{p, q}$ and $\mathbb{C}_{n}$ are isomorphic to direct sums of the two algebras with even dimensionality if correspondingly $p-q \equiv 1,5(\bmod 8)$ and $p+q \equiv 1,3,5,7(\bmod 8)$. Therefore, matrix representations of the algebras $C_{p, q}, \mathbb{C}_{p+q}(p+q=2 m+1)$ are isomorphic to direct sums of the full matrix algebras $\mathrm{M}_{2^{m}}(\mathbb{K}) \oplus \mathrm{M}_{2^{m}}(\mathbb{K})$, where 
$\mathbb{K} \simeq \mathbb{R}, \mathbb{K} \simeq \mathbb{H}, \mathbb{K} \simeq \mathbb{C}$. On the other hand, there exists a homomorphic mapping of the algebras $C \ell_{p, q}$ and $\mathbb{C}_{n}$ onto one matrix algebra $\mathrm{M}_{2^{m}}(\mathbb{K})$ with preservation of addition, multiplication and multiplication by the number operations. Besides, in case of the field $\mathbb{F}=\mathbb{R}$ and $p-q \equiv 3,7(\bmod 8)$ the algebra $C \ell_{p, q}$ is isomorphic to a full matrix algebra $\mathrm{M}_{2^{m}}(\mathbb{C})$, therefore, representations of the fundamental automorphisms of this algebra can be realized by means of $\mathrm{M}_{2^{m}}(\mathbb{C})$.

Theorem 13. If $p+q=2 m+1$, then there exist the following homomorphisms: 1) $\mathbb{F}=\mathbb{R}$

$$
\epsilon: \ell_{p, q} \longrightarrow \mathrm{M}_{2^{m}}(\mathbb{K}),
$$

where $\mathbb{K} \simeq \mathbb{R}$ if $p-q \equiv 1(\bmod 8)$ and $\mathbb{K} \simeq \mathbb{H}$ if $p-q \equiv 5(\bmod 8)$.

2) $\mathbb{F}=\mathbb{C}$

$$
\epsilon: \mathbb{C}_{p+q} \longrightarrow \mathrm{M}_{2^{m}}(\mathbb{C}), \quad \text { if } p-q \equiv 1,3,5,7 \quad(\bmod 8)
$$

Proof. Let us start the proof with a more general case $\mathbb{F}=\mathbb{C}$. In accordance with (44) the element $\omega$ belongs to a center of the algebra $\mathbb{C}_{n}$ and, therefore, this element commutes with all the basis elements of $\mathbb{C}_{n}$. Further, we see that the basis vectors $\left\{e_{1}, e_{2}, \ldots, e_{n}\right\}$ generate a subspace $\mathbb{C}^{n} \subset \mathbb{C}^{n+1}$. Thus, the algebra $\mathbb{C}_{n}$ in $\mathbb{C}^{n}$ is a subalgebra of $\mathbb{C}_{n+1}$ and consists of the elements which do not contain the element $\mathbf{e}_{n+1}$. The each element $\mathcal{A} \in \mathbb{C}_{n+1}$ can be decomposed as follows

$$
\mathcal{A}=\mathcal{A}^{1}+\mathcal{A}^{0},
$$

where the set $\mathcal{A}^{0}$ contains all the elements with $\mathbf{e}_{n+1}$, and $\mathcal{A}^{1}$ does not contain $\mathbf{e}_{n+1}$, therefore, $\mathcal{A}^{1} \in \mathbb{C}_{n}$. Multiplying $\mathcal{A}^{0}$ by $\epsilon \omega$, we see that the elements $\mathbf{e}_{n+1}$ are mutually annihilate. Hence it follows that $\epsilon \omega \mathcal{A}^{0} \in \mathbb{C}_{n}$. Denoting $\mathcal{A}^{2}=\epsilon \omega \mathcal{A}^{0}$ and taking into account that $(\epsilon \omega)^{2}=1$, we obtain

$$
\mathcal{A}=\mathcal{A}^{1}+\varepsilon \omega \mathcal{A}^{2}
$$

where $\mathcal{A}^{1}, \mathcal{A}^{2} \in \mathbb{C}_{n}$. Let us define now a homomorphism $\epsilon: \mathbb{C}_{n+1} \rightarrow \mathbb{C}_{n}$, acting via the following law

$$
\epsilon: \mathcal{A}^{1}+\varepsilon \omega \mathcal{A}^{2} \longrightarrow \mathcal{A}^{1}+\mathcal{A}^{2} .
$$

It is obvious that the operations of addition, multiplication and multiplication by the number are preserved. Indeed, if we take

$$
\mathcal{A}=\mathcal{A}^{1}+\varepsilon \omega \mathcal{A}^{2}, \quad \mathcal{B}=\mathcal{B}^{1}+\mathcal{B}^{2},
$$


then in virtue of $(\varepsilon \omega)^{2}=1$ and commutativity of $\omega$ with all elements we have for the multiplication operation:

$$
\begin{aligned}
\mathcal{A B}=\left(\mathcal{A}^{1} \mathcal{B}^{1}+\right. & \left.\mathcal{A}^{2} \mathcal{B}^{2}\right)+\varepsilon \omega\left(\mathcal{A}^{1} \mathcal{B}^{2}+\mathcal{A}^{2} \mathcal{B}^{1}\right) \stackrel{\epsilon}{\longrightarrow} \\
& \left(\mathcal{A}^{1} \mathcal{B}^{1}+\mathcal{A}^{2} \mathcal{B}^{2}\right)+\left(\mathcal{A}^{1} \mathcal{B}^{2}+\mathcal{A}^{2} \mathcal{B}^{1}\right)=\left(\mathcal{A}^{1}+\mathcal{A}^{2}\right)\left(\mathcal{B}^{1}+\mathcal{B}^{2}\right),
\end{aligned}
$$

that is, an image of the product is equal to the product of factor images in the same order. In particular case at $\mathcal{A}=\varepsilon \omega$ we have $\mathcal{A}^{1}=0$ and $\mathcal{A}^{2}=1$, therefore,

$$
\varepsilon \omega \longrightarrow 1 \text {. }
$$

Thus, a kernel of the homomorphism $\epsilon$ consists of the all elements of the form $\mathcal{A}^{1}-\varepsilon \omega \mathcal{A}^{1}$, which, as it is easy to see, under action of $\epsilon$ are mapped into zero. It is clear that $\operatorname{Ker} \epsilon=\left\{\mathcal{A}^{1}-\varepsilon \omega \mathcal{A}^{1}\right\}$ is a subalgebra of $\mathbb{C}_{n+1}$. Moreover, the kernel of $\epsilon$ is a bilateral ideal of the algebra $\mathbb{C}_{n+1}$. Therefore, the algebra ${ }^{\epsilon} \mathbb{C}_{n}$, obtained in the result of the mapping $\epsilon: \mathbb{C}_{n+1} \rightarrow \mathbb{C}_{n}$, is a quotient algebra on the ideal Ker $\epsilon=\left\{\mathcal{A}^{1}-\varepsilon \omega \mathcal{A}^{1}\right\}$ :

$$
{ }^{\epsilon} \mathbb{C}_{n} \simeq \mathbb{C}_{n+1} / \operatorname{Ker} \epsilon .
$$

Since the algebra $\mathbb{C}_{n}$ at $n=2 m$ is isomorphically mapped onto the full matrix algebra $\mathrm{M}_{2^{m}}(\mathbb{C})$, then in virtue of $\epsilon: \mathbb{C}_{n+1} \rightarrow \mathbb{C}_{n} \subset \mathbb{C}_{n+1}$ we obtain a homomorphic mapping of the algebra $\mathbb{C}_{n+1}$ onto the matrix algebra $\mathrm{M}_{2^{m}}(\mathbb{C})$.

A homomorphism $\epsilon: C \ell_{p, q} \rightarrow \mathrm{M}_{2^{m}}(\mathbb{K})$ is proved analogously. In this case a quotient algebra has the form

$$
{ }^{\epsilon} C \ell_{p, q-1} \simeq C \ell_{p, q} / \text { Ker } \epsilon
$$

or

$$
{ }^{\epsilon} C \ell_{q, p-1} \simeq C \ell_{p, q} / \operatorname{Ker} \epsilon,
$$

where Ker $\epsilon=\left\{\mathcal{A}^{1}-\omega \mathcal{A}^{1}\right\}$, since in accordance with (3) at $p-q \equiv 1,5(\bmod 8)$ we have always $\omega^{2}=1$ and, therefore, $\varepsilon=1$.

As known, complex Clifford algebras $\mathbb{C}_{n}$ are modulo 2 periodic [4] and, therefore, there exist two types of $\mathbb{C}_{n}: n \equiv 0(\bmod 2)$ and $n \equiv 1(\bmod 2)$. We consider these two types in the form of the following series:

$$
\begin{array}{llllllllll}
\mathbb{C}_{2} & & \mathbb{C}_{4} & & \cdots & & \mathbb{C}_{2 k} & & & \\
& \mathbb{C}_{3} & & \mathbb{C}_{5} & & \cdots & & \mathbb{C}_{2 k+1} & & \cdots
\end{array}
$$


Let us study the decomposition $\mathbb{C}_{2 k+1} \simeq \mathbb{C}_{2 k} \oplus \mathbb{C}_{2 k}$ in more details. This decomposition may be represented by a following scheme

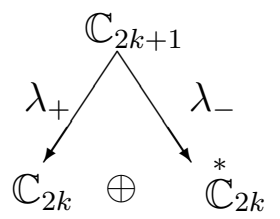

Here central idempotents

$$
\lambda^{+}=\frac{1+\varepsilon \mathbf{e}_{1} \mathbf{e}_{2} \cdots \mathbf{e}_{2 k+1}}{2}, \quad \lambda^{-}=\frac{1-\varepsilon \mathbf{e}_{1} \mathbf{e}_{2} \cdots \mathbf{e}_{2 k+1}}{2},
$$

where

$$
\varepsilon=\left\{\begin{array}{lll}
1, & \text { if } k \equiv 0 & (\bmod 2) \\
i, & \text { if } k \equiv 1 & (\bmod 2),
\end{array}\right.
$$

satisfy the relations $\left(\lambda^{+}\right)^{2}=\lambda^{+},\left(\lambda^{-}\right)^{2}=\lambda^{-}, \lambda^{+} \lambda^{-}=0$. Thus, we have a decomposition of the initial algebra $\mathbb{C}_{2 k+1}$ into a direct sum of two mutually annihilating simple ideals: $\mathbb{C}_{2 k+1} \simeq \frac{1}{2}(1+\varepsilon \omega) \mathbb{C}_{2 k+1} \oplus \frac{1}{2}(1-\varepsilon \omega) \mathbb{C}_{2 k+1}$. Each of the ideals $\lambda^{ \pm} \mathbb{C}_{2 k+1}$ is isomorphic to the subalgebra $\mathbb{C}_{2 k} \subset \mathbb{C}_{2 k+1}$. According to Chisholm and Farwell [22, the idempotents $\lambda^{+}$and $\lambda^{-}$can be identified with the helicity projection operators which distinguish left and right handed spinors. The Chisholm-Farwell notation for $\lambda^{ \pm}$we will widely use below. Therefore, in virtue of the isomorphism $\mathbb{C}_{2 k+1} \simeq \mathbb{C}_{2 k} \cup \mathbb{C}_{2 k}$ and the homomorphic mapping $\epsilon: \mathbb{C}_{2 k+1} \rightarrow \mathbb{C}_{2 k}$, the second series (type $\left.n \equiv 1(\bmod 2)\right)$ is replaced by a sequence of the quotient algebras ${ }^{\epsilon} \mathbb{C}_{2 k}$, that is,

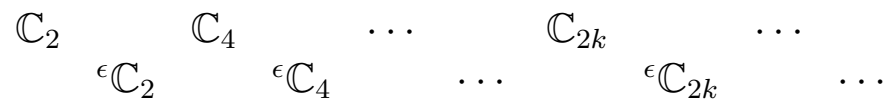

Therefore, all the representations of $\operatorname{Pin}(n, \mathbb{C})$ are divided into the following two series:

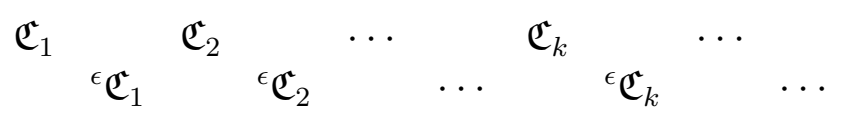

Further, over the field $\mathbb{F}=\mathbb{R}$ for the odd-dimensional algebras $C \ell_{p, q}, p-q \equiv$ $1,5(\bmod 8)$, there exist two different decompositions: $C \ell_{p, q} \simeq C \ell_{p, q-1} \oplus C \ell_{p, q-1}$ and $C \ell_{p, q} \simeq C \ell_{q, p-1} \oplus C \ell_{q, p-1}$. These decompositions can be represented by the 
Table 7. Quotient representations of $\operatorname{Pin}(p, q)$.

\begin{tabular}{|c|c|c|c|c|c|c|c|c|c|c|}
\hline & $\mathrm{p}$ & 0 & 1 & 2 & 3 & 4 & 5 & 6 & 7 & $\ldots$ \\
\hline q & & & & & & & & & & \\
\hline 0 & & $\mathfrak{R}_{0}^{0}$ & ${ }^{\epsilon} \mathfrak{R}_{0}^{0}$ & $\mathfrak{R}_{1}^{2}$ & $\mathfrak{C}_{1}^{3}$ & $\mathfrak{H}_{1}^{4}$ & ${ }^{\epsilon} \mathfrak{H}_{1}^{4}$ & $\mathfrak{H}_{2}^{6}$ & $\mathfrak{C}_{4}^{7}$ & \\
\hline 1 & & $\mathfrak{C}_{0}^{7}$ & $\mathfrak{R}_{1}^{0}$ & ${ }^{\epsilon} \mathfrak{R}_{1}^{0}$ & $\mathfrak{R}_{2}^{2}$ & $\mathfrak{C}_{2}^{3}$ & $\mathfrak{H}_{2}^{4}$ & ${ }^{\epsilon} \mathfrak{H}_{2}^{4}$ & $\mathfrak{H}_{4}^{6}$ & \\
\hline 2 & & $\mathfrak{H}_{0}^{6}$ & $\mathfrak{C}_{1}^{7}$ & $\mathfrak{R}_{2}^{0}$ & ${ }^{\epsilon} \mathfrak{R}_{2}^{0}$ & $\mathfrak{R}_{4}^{2}$ & $\mathfrak{C}_{4}^{3}$ & $\mathfrak{H}_{4}^{4}$ & ${ }^{\epsilon} \mathfrak{H}_{4}^{4}$ & \\
\hline 3 & & ${ }^{\epsilon} \mathfrak{H}_{0}^{4}$ & $\mathfrak{H}_{1}^{6}$ & $\mathfrak{C}_{2}^{7}$ & $\mathfrak{R}_{4}^{0}$ & $\epsilon \mathfrak{R}_{4}^{0}$ & $\mathfrak{R}_{8}^{2}$ & $\mathfrak{C}_{8}^{3}$ & $\mathfrak{H}_{8}^{4}$ & \\
\hline 4 & & $\mathfrak{H}_{1}^{4}$ & ${ }^{\epsilon} \mathfrak{H}_{1}^{4}$ & $\mathfrak{H}_{2}^{6}$ & $\mathfrak{C}_{4}^{7}$ & $\mathfrak{R}_{8}^{0}$ & $\epsilon \mathfrak{R}_{8}^{0}$ & $\mathfrak{R}_{16}^{2}$ & $\mathfrak{C}_{16}^{3}$ & \\
\hline 5 & & $\mathfrak{C}_{2}^{3}$ & $\mathfrak{H}_{2}^{4}$ & ${ }^{\epsilon} \mathfrak{H}_{2}^{4}$ & $\mathfrak{H}_{4}^{6}$ & $\mathfrak{C}_{8}^{7}$ & $\mathfrak{R}_{16}^{0}$ & ${ }^{\epsilon} \mathfrak{R}_{16}^{0}$ & $\mathfrak{R}_{32}^{2}$ & \\
\hline 6 & & $\mathfrak{R}_{4}^{2}$ & $\mathfrak{C}_{4}^{3}$ & $\mathfrak{H}_{4}^{4}$ & ${ }^{\epsilon} \mathfrak{H}_{4}^{4}$ & $\mathfrak{H}_{8}^{6}$ & $\mathfrak{C}_{16}^{7}$ & $\mathfrak{R}_{32}^{0}$ & ${ }^{\epsilon} \mathfrak{R}_{32}^{0}$ & \\
\hline 7 & & $\epsilon \mathfrak{R}_{4}^{0}$ & $\mathfrak{R}_{8}^{2}$ & $\mathfrak{C}_{8}^{3}$ & $\mathfrak{H}_{8}^{4}$ & ${ }^{\epsilon} \mathfrak{H}_{8}^{4}$ & $\mathfrak{H}_{16}^{6}$ & $\mathfrak{C}_{32}^{7}$ & $\mathfrak{R}_{64}^{0}$ & \\
\hline$\vdots$ & & $\vdots$ & : & $\vdots$ & $\vdots$ & $\vdots$ & $\vdots$ & $\vdots$ & $\vdots$ & \\
\hline
\end{tabular}

following schemes:
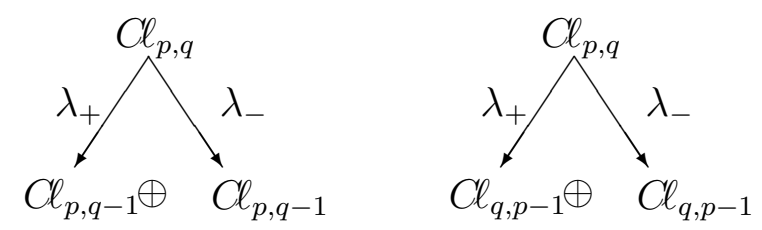

Here central idempotents

$$
\lambda^{+}=\frac{1+\mathbf{e}_{1} \mathbf{e}_{2} \cdots \mathbf{e}_{p+q}}{2}, \quad \lambda^{-}=\frac{1-\mathbf{e}_{1} \mathbf{e}_{2} \cdots \mathbf{e}_{p+q}}{2}
$$

satisfy the relations $\left(\lambda^{+}\right)^{2}=\lambda^{+},\left(\lambda^{-}\right)^{2}=\lambda^{-}, \lambda^{+} \lambda^{-}=0$. Therefore, in virtue of the homomorphic mappings $\epsilon: C \ell_{p, q} \rightarrow{ }^{\epsilon} C \ell_{p, q-1}$ and $\epsilon: C \ell_{p, q} \rightarrow{ }^{\epsilon} C_{q, p-1}$ we can replace the double representations ${ }^{2} \mathfrak{R},{ }^{2} \mathfrak{H}$ by quotient representations ${ }^{\epsilon} \mathfrak{R}$, ${ }^{\epsilon} \mathfrak{H}$. The distribution of the quotient representations of $\operatorname{Pin}(p, q)$ is given in the Table 7.

\section{Fundamental automorphisms of odd-dimensional Clifford algebras}

Let us consider now the automorphisms $\mathcal{A} \rightarrow \mathcal{A}^{\star}, \mathcal{A} \rightarrow \widetilde{\mathcal{A}}, \mathcal{A} \rightarrow \widetilde{\mathcal{A}^{\star}}, \mathcal{A} \rightarrow \overline{\mathcal{A}}$, $\mathcal{A} \rightarrow \overline{\mathcal{A}^{\star}}, \mathcal{A} \rightarrow \overline{\widetilde{\mathcal{A}}}, \mathcal{A} \rightarrow \overline{\widetilde{\mathcal{A}^{\star}}}$ of the algebras $\mathbb{C}_{n+1}$ and $C_{p, q}, p-q \equiv 1,3,5,7$ 
(mod 8). We will examine the form of the fundamental automorphisms defined in $\mathbb{C}_{n+1}$ and $C \ell_{p, q}$ after the homomorphic mappings $\epsilon: \mathbb{C}_{n+1} \rightarrow \mathbb{C}_{n} \subset \mathbb{C}_{n+1}$ and $\epsilon: C \ell_{p, q} \rightarrow{ }^{\epsilon} C \ell_{p, q-1}, \epsilon: C \ell_{p, q} \rightarrow{ }^{\epsilon} C \ell_{q, p-1}$.

Theorem 14. 1) $\mathbb{F}=\mathbb{C}$. Let $\mathcal{A} \rightarrow \overline{\mathcal{A}}, \mathcal{A} \rightarrow \mathcal{A}^{\star}, \mathcal{A} \rightarrow \widetilde{\mathcal{A}}$ be the automorphisms of the odd-dimensional complex Clifford algebra $\mathbb{C}_{n+1}(n+1 \equiv 1,3(\bmod 4))$ corresponding the discrete transformations $C, P, T$ (charge conjugation, space inversion, time reversal) and let ${ }^{\epsilon} \mathbb{C}_{n}$ be a quotient algebra obtained in the result of the homomorphic mapping $\epsilon: \mathbb{C}_{n+1} \rightarrow \mathbb{C}_{n}$. Then over the field $\mathbb{F}=\mathbb{C}$ in dependence on the structure of $\mathbb{C}_{n}$ all the quotient representations of the group $\operatorname{Pin}(p, q)$ are divided in the following six classes:

$$
\begin{aligned}
\text { 1) }{ }^{\epsilon} \mathfrak{C}_{a_{1}} & :\{T, C \sim I\}, \\
2)^{\epsilon} \mathfrak{C}_{a_{2}} & :\{T, C\}, \\
3){ }^{\epsilon} \mathfrak{C}_{b}: & : T T, C P, C P T\}, \\
\text { 4) }{ }^{\epsilon} \mathfrak{C}_{c} & :\{P T, C, C P T\}, \\
\text { 5) }{ }^{\epsilon} \mathfrak{C}_{d_{1}}: & :\{P T, C P \sim I P, C T \sim I T\}, \\
6)^{\epsilon} \mathfrak{C}_{d_{2}}: & :\{P T, C P, C T\} .
\end{aligned}
$$

2) $\mathbb{F}=\mathbb{R}$. Real quotient representations are divided into four different classes:

$$
\begin{aligned}
\text { 7) }{ }^{\epsilon} \mathfrak{R}_{e_{1}} & : \quad\{T, C \sim I, C T \sim I T\}, \\
8)^{\epsilon} \mathfrak{R}_{e_{2}} & : \quad\{T, C P \sim I P, C P T \sim I P T\}, \\
9)^{\epsilon} \mathfrak{H}_{f_{1}}: & :\left\{T, C \sim C^{\prime}, C T \sim C^{\prime} T\right\}, \\
10)^{\epsilon} \mathfrak{H}_{f_{2}} & : \quad\left\{T, C P \sim C^{\prime} P, C P T \sim C^{\prime} P T\right\} .
\end{aligned}
$$

Proof. 1) Complex representations.

Before we proceed to find an explicit form of the quotient representations ${ }^{\epsilon} \mathfrak{C}$ it is necessary to consider in details a structure of the quotient algebras ${ }^{\epsilon} \mathbb{C}_{n}$ obtained in the result of the homomorphic mapping $\epsilon: \mathbb{C}_{n+1} \rightarrow \mathbb{C}_{n}$. The structure of the quotient algebra ${ }^{\epsilon} \mathbb{C}_{n}$ depends on the transfer of the automorphisms $\mathcal{A} \rightarrow \mathcal{A}^{\star}, \mathcal{A} \rightarrow \widetilde{\mathcal{A}}, \mathcal{A} \rightarrow \widetilde{\mathcal{A}^{\star}}, \mathcal{A} \rightarrow \overline{\mathcal{A}}, \mathcal{A} \rightarrow \overline{\mathcal{A}^{\star}}, \mathcal{A} \rightarrow \overline{\widetilde{\mathcal{A}}}, \mathcal{A} \rightarrow \overline{\widetilde{\mathcal{A}^{\star}}}$ of the algebra $\mathbb{C}_{n+1}$ onto its subalgebra $\mathbb{C}_{n}$ under action of the homomorphism $\epsilon$. The action of the homomorphism $\epsilon$ is defined as follows

$$
\epsilon: \mathcal{A}^{1}+\varepsilon \omega \mathcal{A}^{2} \longrightarrow \mathcal{A}^{1}+\mathcal{A}^{2},
$$

where $\mathcal{A}^{1}, \mathcal{A}^{2} \in \mathbb{C}_{n}, \omega=\mathbf{e}_{12 \cdots n+1}$, and

$$
\varepsilon= \begin{cases}1, & \text { if } n+1 \equiv 1(\bmod 4) \\ i, & \text { if } n+1 \equiv 3(\bmod 4)\end{cases}
$$


so that $(\varepsilon \omega)^{2}=1$. At this point, $\varepsilon \omega \rightarrow 1$, and the quotient algebra has a form

$$
{ }^{\epsilon} \mathbb{C}_{n} \simeq \mathbb{C}_{n+1} / \operatorname{Ker} \epsilon,
$$

where $\operatorname{Ker} \epsilon=\left\{\mathcal{A}^{1}-\varepsilon \omega \mathcal{A}^{1}\right\}$ is a kernel of the homomorphism $\epsilon$.

First of all, for the antiautomorphism $\mathcal{A} \rightarrow \widetilde{\mathcal{A}}$ we see that elements $\mathcal{A}, \mathcal{B}, \ldots \in \mathbb{C}_{n+1}$, which are mapped into one and the same element $\mathcal{D} \in \mathbb{C}_{n}$ (a kernel of the homomorphism $\epsilon$ if $\mathcal{D}=0$ ), after the transformation $\mathcal{A} \rightarrow \widetilde{\mathcal{A}}$ are must converted to the elements $\widetilde{\mathcal{A}}, \widetilde{\mathcal{B}}, \ldots \in \mathbb{C}_{n+1}$, which are also mapped into one and the same element $\widetilde{\mathcal{D}} \in \mathbb{C}_{n}$. Otherwise, the transformation $\mathcal{A} \rightarrow \widetilde{\mathcal{A}}$ is not transferred from $\mathbb{C}_{n+1}$ to $\mathbb{C}_{n}$ as an unambiguous transformation. Therefore, for the transfer of the antiautomorphism $\mathcal{A} \rightarrow \widetilde{\mathcal{A}}$ from $\mathbb{C}_{n+1}$ into $\mathbb{C}_{n}$ it is necessary that

$$
\widetilde{\varepsilon \omega}=\varepsilon \omega .
$$

Indeed, since under action of $\epsilon$ the elements 1 and $\varepsilon \omega$ are equally mapped into the unit, then transformed elements $\widetilde{1}$ and $\widetilde{\varepsilon \omega}$ are also should be mapped into 1 , but $\widetilde{1}=1 \rightarrow 1$, and $\widetilde{\varepsilon \omega}= \pm \varepsilon \omega \rightarrow \pm 1$ in virtue of $\widetilde{\omega}=(-1)^{\frac{n(n-1)}{2} \omega} \omega$, whence

$$
\widetilde{\omega}= \begin{cases}\omega, & \text { if } n+1 \equiv 1(\bmod 4) ; \\ -\omega, & \text { if } n+1 \equiv 3(\bmod 4) .\end{cases}
$$

The condition (105) is sufficient for the transfer of the antiautomorphism $\mathcal{A} \rightarrow$ $\widetilde{\mathcal{A}}$ from $\mathbb{C}_{n+1}$ to $\mathbb{C}_{n}$. Indeed, in this case at $\mathcal{A} \rightarrow \widetilde{\mathcal{A}}$ we have

$$
\mathcal{A}^{1}-\mathcal{A}^{1} \varepsilon \omega \longrightarrow \widetilde{\mathcal{A}^{1}}-\widetilde{\varepsilon \omega} \widetilde{\mathcal{A}^{1}}=\widetilde{\mathcal{A}^{1}}-\varepsilon \omega \widetilde{\mathcal{A}^{1}} .
$$

Thus, elements of the form $\mathcal{A}^{1}-\mathcal{A}^{1} \varepsilon \omega$, consisting of the kernel of the homomorphism $\epsilon$, are converted at the transformation $\mathcal{A} \rightarrow \widetilde{\mathcal{A}}$ into elements of the same form. Therefore, under action of the homomorphism $\epsilon$ the antiautomorphism $\mathcal{A} \rightarrow \widetilde{\mathcal{A}}$ is transferred from $\mathbb{C}_{n+1}$ into $\mathbb{C}_{n}$ only at $n \equiv 0(\bmod 4)$.

In turn, for the transfer of the automorphism $\mathcal{A} \rightarrow \mathcal{A}^{\star}$ it is necessary that $(\varepsilon \omega)^{\star}=\varepsilon \omega$. However, since the element $\omega$ is odd and $\omega^{\star}=(-1)^{n+1} \omega$, then we have always

$$
\omega^{\star}=-\omega .
$$

Thus, the automorphism $\mathcal{A} \rightarrow \mathcal{A}^{\star}$ is never transferred from $\mathbb{C}_{n+1}$ into $\mathbb{C}_{n}$.

Further, for the transfer of the antiautomorphism $\mathcal{A} \rightarrow \widetilde{\mathcal{A}^{\star}}$ from $\mathbb{C}_{n+1}$ into $\mathbb{C}_{n}$ it is necessary that

$$
\widetilde{(\varepsilon \omega)^{\star}}=\varepsilon \omega \text {. }
$$


It is easy to see that the condition (108) is satisfied only at $n+1 \equiv 3(\bmod 4)$, since in this case from the second equality of (106) and (107) it follows

$$
\widetilde{(\varepsilon \omega)^{\star}}=\widetilde{\varepsilon \omega^{\star}}=-\varepsilon \omega^{\star}=\varepsilon \omega .
$$

Therefore, under action of the homomorphism $\epsilon$ the antiautomorphism $\mathcal{A} \rightarrow$ $\widetilde{\mathcal{A}^{\star}}$ is transferred from $\mathbb{C}_{n+1}$ into $\mathbb{C}_{n}$ only at $n \equiv 2(\bmod 4)$.

Let $n+1=p+q$. Defining in $\mathbb{C}_{n+1}$ the basis $\left\{\mathbf{e}_{1}, \ldots, \mathbf{e}_{p}, i \mathbf{e}_{p+1}, \ldots, i \mathbf{e}_{p+q}\right\}$, we extract the real subalgebra $C_{p, q}$, where at $p-q \equiv 3,7(\bmod 8)$ we have a complex division ring $\mathbb{K} \simeq \mathbb{C}$, and at $p-q \equiv 1(\bmod 8)$ and $p-q \equiv 5$ $(\bmod 8)$ correspondingly a double real division $\operatorname{ring} \mathbb{K} \simeq \mathbb{R} \oplus \mathbb{R}$ and a double quaternionic division ring $\mathbb{K} \simeq \mathbb{H} \oplus \mathbb{H}$. The product $\mathbf{e}_{1} \mathbf{e}_{2} \cdots \mathbf{e}_{p} i \mathbf{e}_{p+1} \cdots i \mathbf{e}_{p+q}=$ $i^{q} \omega \in \mathbb{C}_{n+1}$ sets a volume element of the real subalgebra $C \ell_{p, q}$. At this point we have a condition $\overline{\left(i^{q} \omega\right)}=i^{q} \omega$, that is, $(-i)^{q} \bar{\omega}=i^{q} \omega$, whence

$$
\bar{\omega}=(-1)^{q} \omega
$$

When $q$ is even, from (110) it follows that $\bar{\omega}=\omega$ and, therefore, the pseudoautomorphism $\mathcal{A} \rightarrow \overline{\mathcal{A}}$ is transferred at $q \equiv 0(\bmod 2)$, and since $p+q$ is odd number, then we have always $p \equiv 1(\bmod 2)$. In more detail, at $n+1 \equiv 3$ $(\bmod 4)$ the pseudoautomorphism $\mathcal{A} \rightarrow \overline{\mathcal{A}}$ is transferred from $\mathbb{C}_{n+1}$ into $\mathbb{C}_{n}$ if the real subalgebra $C_{p, q}$ possesses the complex ring $\mathbb{K} \simeq \mathbb{C}, p-q \equiv 3,7$ $(\bmod 8)$, and is not transferred $(\bar{\omega}=-\omega, q \equiv 1(\bmod 2), p \equiv 0(\bmod 2))$ in the case of $C \ell_{p, q}$ with double rings $\mathbb{K} \simeq \mathbb{R} \oplus \mathbb{R}$ and $\mathbb{K} \simeq \mathbb{H} \oplus \mathbb{H}, p-q \equiv 1,5$ $(\bmod 8)$. In turn, at $n+1 \equiv 1(\bmod 4)$ the pseudoautomorphism $\mathcal{A} \rightarrow \overline{\mathcal{A}}$ is transferred from $\mathbb{C}_{n+1}$ into $\mathbb{C}_{n}$ if the subalgebra $C_{p, q}$ has the type $p-q \equiv 1,5$ $(\bmod 8)$ and is not transferred in the case of $C \ell_{p, q}$ with $p-q \equiv 3,7(\bmod 8)$. Besides, in virtue of (107) at $n+1 \equiv 3(\bmod 4)$ with $p-q \equiv 1,5(\bmod 8)$ and at $n+1 \equiv 1(\bmod 4)$ with $p-q \equiv 3,7(\bmod 8)$ a pseudoautomorphism $\mathcal{A} \rightarrow \overline{\mathcal{A}^{\star}}$ (a composition of the pseudoautomorphism $\mathcal{A} \rightarrow \overline{\mathcal{A}}$ with the automorphism $\left.\mathcal{A} \rightarrow \mathcal{A}^{\star}\right)$ is transferred from $\mathbb{C}_{n+1}$ into $\mathbb{C}_{n}$, since

$$
\overline{\varepsilon \omega^{\star}}=\varepsilon \omega \text {. }
$$

Further, in virtue of the second equality of (106) at $n+1 \equiv 3(\bmod 4)$ with $p-q \equiv 1,5(\bmod 8)$ a pseudoantiautomorphism $\mathcal{A} \rightarrow \overline{\widetilde{\mathcal{A}}}$ (a composition of the pseudoautomorphism $\mathcal{A} \rightarrow \overline{\mathcal{A}}$ with the antiautomorphism $\mathcal{A} \rightarrow \widetilde{\mathcal{A}}$ ) is transferred from $\mathbb{C}_{n+1}$ into $\mathbb{C}_{n}$, since

$$
\overline{\widetilde{\varepsilon \omega}}=\varepsilon \omega \text {. }
$$


Finally, a pseudoantiautomorphism $\mathcal{A} \rightarrow \overline{\widetilde{\mathcal{A}^{\star}}}$ (a composition of the pseudoautomorphism $\mathcal{A} \rightarrow \overline{\mathcal{A}}$ with the antiautomorphism $\left.\mathcal{A} \rightarrow \widetilde{\mathcal{A}^{\star}}\right)$, corresponded to $C P T$-transformation (see Proposition 2), is transferred from $\mathbb{C}_{n+1}$ into $\mathbb{C}_{n}$ at $n+1 \equiv 3(\bmod 4)$ and $C \ell_{p, q}$ with $p-q \equiv 3,7(\bmod 8)$, since in this case in virtue of (109) and (110) we have

$$
\overline{\left(\varepsilon \omega^{\star}\right)}=\varepsilon \omega
$$

Also at $n+1 \equiv 1(\bmod 4)$ and $q \equiv 1(\bmod 2)$ we obtain

$$
\left.\widetilde{\left.\overline{\varepsilon \omega^{\star}}\right)}=-\widetilde{\varepsilon \omega^{\star}}\right)=-(\varepsilon \omega)^{\star}=\varepsilon \omega
$$

therefore, the transformation $\mathcal{A} \rightarrow \overline{\widetilde{\mathcal{A}^{\star}}}$ is transferred at $n+1 \equiv 1(\bmod 4)$ and $C \ell_{p, q}$ with $p-q \equiv 3,7(\bmod 8)$.

The conditions for the transfer of the fundamental automorphisms of the algebra $\mathbb{C}_{n+1}$ into its subalgebra $\mathbb{C}_{n}$ under action of the homomorphism $\epsilon$ allow us to define in evident way an explicit form of the quotient algebras ${ }^{\epsilon} \mathbb{C}_{n}$.

1) The quotient algebra ${ }^{\epsilon} \mathbb{C}_{n}, n \equiv 0(\bmod 4)$.

As noted previously, in the case $n+1 \equiv 1(\bmod 4)$ the antiautomorphism $\mathcal{A} \rightarrow \widetilde{\mathcal{A}}$ and pseudoautomorphism $\mathcal{A} \rightarrow \overline{\mathcal{A}}$ are transferred from $\mathbb{C}_{n+1}$ into $\mathbb{C}_{n}$ if the subalgebra $C_{p, q} \subset \mathbb{C}_{n+1}$ possesses the double rings $\mathbb{K} \simeq \mathbb{R} \oplus \mathbb{R}$, $\mathbb{K} \simeq \mathbb{H} \oplus \mathbb{H}(p-q \equiv 1,5(\bmod 8))$, and also the pseudoautomorphism $\mathcal{A} \rightarrow \overline{\mathcal{A}^{\star}}$ and pseudoantiautomorphism $\mathcal{A} \rightarrow \overline{\widetilde{\mathcal{A}^{\star}}}$ are transferred if $C \ell_{p, q}$ has the complex ring $\mathbb{K} \simeq \mathbb{C}(p-q \equiv 3,7(\bmod 8))$. It is easy to see that in dependence on the type of $C_{p, q}$ the structure of the quotient algebras ${ }^{\epsilon} \mathbb{C}_{n}$ of this type is divided into two different classes:

a) The class of quotient algebras ${ }^{\epsilon} \mathbb{C}_{n}$ containing the antiautomorphism $\mathcal{A} \rightarrow \widetilde{\mathcal{A}}$ and pseudoautomorphism $\mathcal{A} \rightarrow \overline{\mathcal{A}}$. It is obvious that in dependence on a division ring structure of the subalgebra $C \ell_{p, q} \subset \mathbb{C}_{n+1}$ this class is divided into two subclasses:

$\left.a_{1}\right){ }^{\epsilon} \mathbb{C}_{n}$ with $\mathcal{A} \rightarrow \widetilde{\mathcal{A}}, \mathcal{A} \rightarrow \overline{\mathcal{A}}$ when $C \ell_{p, q}$ has the $\operatorname{ring} \mathbb{K} \simeq \mathbb{R} \oplus \mathbb{R}, p-q \equiv 1$ $(\bmod 8)$.

$\left.a_{2}\right) \epsilon_{\mathbb{C}_{n}}$ with $\mathcal{A} \rightarrow \widetilde{\mathcal{A}}, \mathcal{A} \rightarrow \overline{\mathcal{A}}$ when $C_{p, q}$ has the $\operatorname{ring} \mathbb{K} \simeq \mathbb{H} \oplus \mathbb{H}, p-q \equiv 5$ $(\bmod 8)$.

b) The class of quotient algebras ${ }^{\epsilon} \mathbb{C}_{n}$ containing the transformations $\mathcal{A} \rightarrow \widetilde{\mathcal{A}}$, $\mathcal{A} \rightarrow \overline{\mathcal{A}^{\star}}, \mathcal{A} \rightarrow \overline{\widetilde{\mathcal{A}^{\star}}}$ if the subalgebra $C_{p, q} \subset \mathbb{C}_{n+1}$ has the complex ring $\mathbb{K} \simeq \mathbb{C}$, 
$p-q \equiv 3,7(\bmod 8)$.

2) The quotient algebra ${ }^{\epsilon} \mathbb{C}_{n}, n \equiv 2(\bmod 4)$.

In the case $n+1 \equiv 3(\bmod 4)$ the antiautomorphism $\mathcal{A} \rightarrow \widetilde{\mathcal{A}^{\star}}$, pseudoautomorphism $\mathcal{A} \rightarrow \overline{\mathcal{A}}$ and pseudoantiautomorphism $\mathcal{A} \rightarrow \overline{\widetilde{\mathcal{A}^{\star}}}$ are transferred from $\mathbb{C}_{n+1}$ into $\mathbb{C}_{n}$ if the subalgebra $C \ell_{p, q} \subset \mathbb{C}_{n+1}$ possesses the complex ring $\mathbb{K} \simeq \mathbb{C}(p-q \equiv 3,7(\bmod 8))$, and also the pseudoautomorphism $\mathcal{A} \rightarrow \overline{\mathcal{A}^{\star}}$ and pseudoantiautomorphism $\mathcal{A} \rightarrow \overline{\widetilde{\mathcal{A}}}$ are transferred if $C \ell_{p, q}$ has the double rings $\mathbb{K} \simeq \mathbb{R} \oplus \mathbb{R}, \mathbb{K} \simeq \mathbb{H} \oplus \mathbb{H}(p-q \equiv 1,5(\bmod 8))$. In dependence on the type of $C_{p, q} \subset \mathbb{C}_{n+1}$ all the quotient algebras ${ }^{\epsilon} \mathbb{C}_{n}$ of this type are divided into following two classes:

c) The class of quotient algebras ${ }^{\epsilon} \mathbb{C}_{n}$ containing the transformations $\mathcal{A} \rightarrow \widetilde{\mathcal{A}^{\star}}$, $\mathcal{A} \rightarrow \overline{\mathcal{A}}, \mathcal{A} \rightarrow \overline{\widetilde{\mathcal{A}^{\star}}}$ if the subalgebra $C \ell_{p, q}$ has the ring $\mathbb{K} \simeq \mathbb{C}, p-q \equiv 3,7$ $(\bmod 8)$.

d) The class of quotient algebras ${ }^{\epsilon} \mathbb{C}_{n}$ containing the antiautomorphism $\mathcal{A} \rightarrow$ $\widetilde{\mathcal{A}^{\star}}$, pseudoautomorphism $\mathcal{A} \rightarrow \overline{\mathcal{A}^{\star}}$ and pseudoautomorphism $\mathcal{A} \rightarrow \overline{\widetilde{\mathcal{A}}}$. At this point, in dependence on the division ring structure of $C_{p, q}$ we have two subclasses

$\left.d_{1}\right){ }^{\epsilon} \mathbb{C}_{n}$ with $\mathcal{A} \rightarrow \widetilde{\mathcal{A}^{\star}}, \mathcal{A} \rightarrow \overline{\mathcal{A}^{\star}}$ and $\mathcal{A} \rightarrow \overline{\widetilde{\mathcal{A}}}$ at $C \ell_{p, q}$ with the ring $\mathbb{K} \simeq \mathbb{R} \oplus \mathbb{R}$, $p-q \equiv 1(\bmod 8)$.

$\left.d_{2}\right){ }^{\epsilon} \mathbb{C}_{n}$ with $\mathcal{A} \rightarrow \widetilde{\mathcal{A}^{\star}}, \mathcal{A} \rightarrow \overline{\mathcal{A}^{\star}}$ and $\mathcal{A} \rightarrow \overline{\widetilde{\mathcal{A}}}$ at $C l_{p, q}$ with the ring $\mathbb{K} \simeq \mathbb{H} \oplus \mathbb{H}$, $p-q \equiv 5(\bmod 8)$.

Thus, we have 6 different classes of the quotient algebras ${ }^{\epsilon} \mathbb{C}_{n}$. Further, in accordance with Proposition [1, the automorphism $\mathcal{A} \rightarrow \mathcal{A}^{\star}$ corresponds to space inversion $P$, the antiautomorphisms $\mathcal{A} \rightarrow \widetilde{\mathcal{A}}$ and $\mathcal{A} \rightarrow \widetilde{\mathcal{A}^{\star}}$ set correspondingly time reversal $T$ and full reflection $P T$, and in accordance with Theorem 3 the pseudoautomorphism $\mathcal{A} \rightarrow \overline{\mathcal{A}}$ corresponds to charge conjugation $C$. Taking into account these relations we come to the classification presented in Theorem for complex quotient representations.

2) Real representations.

Let us define real quotient representations of the group $\operatorname{Pin}(p, q)$. First of all, in the case of types $p-q \equiv 3,7(\bmod 8)$ these representations are equivalent to complex representations. Further, when $p-q \equiv 1,5(\bmod 8)$ we have the real algebras $C \ell_{p, q}$ with the rings $\mathbb{K} \simeq \mathbb{R} \oplus \mathbb{R}, \mathbb{K} \simeq \mathbb{H} \oplus \mathbb{H}$ and, therefore, there exist homomorphic mappings $\epsilon: C \ell_{p, q} \rightarrow C \ell_{p, q-1}, \epsilon: C \ell_{p, q} \rightarrow C_{q, p-1}$. In this 
case the quotient algebra has a form

$$
{ }^{\epsilon} C \ell_{p, q-1} \simeq C \ell_{p, q} / \operatorname{Ker} \epsilon
$$

or

$$
{ }^{\epsilon} C \ell_{q, p-1} \simeq C \ell_{p, q} / \operatorname{Ker} \epsilon
$$

where $\operatorname{Ker} \epsilon=\left\{\mathcal{A}^{1}-\omega \mathcal{A}^{1}\right\}$ is a kernel of $\epsilon$, since in accordance with

$$
\omega^{2}=\left\{\begin{array}{lll}
-1 & \text { if } p-q \equiv 2,3,6,7 \quad(\bmod 8) \\
+1 & \text { if } p-q \equiv 0,1,4,5 \quad(\bmod 8)
\end{array}\right.
$$

at $p-q \equiv 1,5(\bmod 8)$ we have always $\omega^{2}=1$ and, therefore, $\varepsilon=1$. Thus, for the transfer of the antiautomorphism $\mathcal{A} \rightarrow \widetilde{\mathcal{A}}$ from $C \ell_{p, q}$ into $C \ell_{p, q-1}\left(C \ell_{q, p-1}\right)$ it is necessary that

$$
\widetilde{\omega}=\omega .
$$

In virtue of the relation $\widetilde{\omega}=(-1)^{\frac{(p+q)(p+q+1)}{2}} \omega$ we obtain

$$
\widetilde{\omega}=\left\{\begin{array}{lll}
+\omega & \text { if } p-q \equiv 1,5 \quad(\bmod 8), \\
-\omega & \text { if } p-q \equiv 3,7 \quad(\bmod 8)
\end{array}\right.
$$

Therefore, for the algebras over the field $\mathbb{F}=\mathbb{R}$ the antiautomorphism $\mathcal{A} \rightarrow \widetilde{\mathcal{A}}$ is transferred at the mappings $C \ell_{p, q} \rightarrow C \ell_{p, q-1}, C \ell_{p, q} \rightarrow C \ell_{q, p-1}$, where $p-q \equiv$ 1,5 $(\bmod 8)$.

In turn, for the transfer of the automorphism $\mathcal{A} \rightarrow \mathcal{A}^{\star}$ it is necessary that $\omega^{\star}=\omega$. However, since the element $\omega$ is odd and $\omega^{\star}=(-1)^{p+q} \omega$, then we have always

$$
\omega^{\star}=-\omega
$$

Thus, the automorphism $\mathcal{A} \rightarrow \mathcal{A}^{\star}$ is never transferred from $C \ell_{p, q}$ into $C \ell_{p, q-1}$ $\left(C \ell_{q, p-1}\right)$.

Further, for the transfer of the antiautomorphism $\mathcal{A} \rightarrow \widetilde{\mathcal{A}^{\star}}$ it is necessary that

$$
\widetilde{\omega^{\star}}=\omega .
$$

From (111) and (112) for the types $p-q \equiv 1,5(\bmod 8)$ we obtain

$$
\widetilde{\omega^{\star}}=\omega^{\star}=-\omega \text {. }
$$

Therefore, under action of the homomorphism $\epsilon$ the antiautomorphism $\mathcal{A} \rightarrow$ $\widetilde{\mathcal{A}^{\star}}$ is never transferred from $C \ell_{p, q}$ into $C \ell_{p, q-1}\left(C \ell_{q, p-1}\right)$. 
As noted previously, for the real representations of $\operatorname{Pin}(p, q)$ the pseudoautomorphism $\mathcal{A} \rightarrow \overline{\mathcal{A}}$ is reduced into identical transformation I for $\mathfrak{R}_{m}^{0,2}$ and to particle-antiparticle conjugation $C^{\prime}$ for $\mathfrak{H}_{m}^{4,6}$. The volume element $\omega$ of $C \ell_{p, q}$ (types $\left.p-q \equiv 1,5(\bmod 8)\right)$ can be represented by the product $\mathbf{e}_{1} \mathbf{e}_{2} \cdots \mathbf{e}_{p} \mathbf{e}_{p+1}^{\prime} \mathbf{e}_{p+2}^{\prime} \cdots \mathbf{e}_{p+q}^{\prime}$, where $\mathbf{e}_{p+j}^{\prime}=i \mathbf{e}_{p+j}, \mathbf{e}_{j}^{2}=1,\left(\mathbf{e}_{p+j}^{\prime}\right)^{2}=-1$. Therefore, for the transfer of $\mathcal{A} \rightarrow \overline{\mathcal{A}}$ from $C \ell_{p, q}$ into $C \ell_{p, q-1}\left(C \ell_{q, p-1}\right)$ we have a condition

$$
\bar{\omega}=\omega,
$$

and in accordance with (110) it follows that the pseudoautomorphism $\mathcal{A} \rightarrow \overline{\mathcal{A}}$ is transferred at $q \equiv 0(\bmod 2)$. Further, in virtue of the relation (112) the pseudoautomorphism $\mathcal{A} \rightarrow \overline{\mathcal{A}^{\star}}$ is transferred at $q \equiv 1(\bmod 2)$, since in this case we have

$$
\overline{\omega^{\star}}=\omega .
$$

Also from (111) it follows that the pseudoantiautomorphism $\mathcal{A} \rightarrow \overline{\widetilde{\mathcal{A}}}$ is transferred at $p-q \equiv 1,5(\bmod 8)$ and $q \equiv 0(\bmod 2)$, since

$$
\overline{\widetilde{\omega}}=\omega .
$$

Finally, the pseudoantiautomorphism $\mathcal{A} \rightarrow \overline{\widetilde{\mathcal{A}^{\star}}}$ (CPT-transformation) in virtue of (113) and (110) is transferred from $C \ell_{p, q}$ into $C \ell_{p, q-1}\left(C_{q, p-1}\right)$ at $p-q \equiv 1,5(\bmod 8)$ and $q \equiv 1(\bmod 2)$.

Now we are in a position that allows us to classify the real quotient algebras ${ }^{\epsilon} C \ell_{p, q-1}\left({ }^{\epsilon} C_{q, p-1}\right)$.

1) The quotient algebra ${ }^{\epsilon} C \ell_{p, q-1}\left({ }^{\epsilon} C \ell_{q, p-1}\right), p-q \equiv 1(\bmod 8)$.

In this case the initial algebra $C \ell_{p, q}$ has the double real division ring $\mathbb{K} \simeq \mathbb{R} \oplus \mathbb{R}$ and its subalgebras $C \ell_{p, q-1}$ and $C \ell_{q, p-1}$ are of the type $p-q \equiv 0(\bmod 8)$ or $p-q \equiv 2(\bmod 8)$ with the ring $\mathbb{K} \simeq \mathbb{R}$. Therefore, in accordance with Theorem 3 for all such quotient algebras the pseudoautomorphism $\mathcal{A} \rightarrow \overline{\mathcal{A}}$ is equivalent to the identical transformation I. The antiautomorphism $\mathcal{A} \rightarrow \widetilde{\mathcal{A}}$ in this case is transferred into $C \ell_{p, q-1}\left(C \ell_{q, p-1}\right)$ at any $p-q \equiv 1(\bmod 8)$. Further, in dependence on the number $q$ we have two different classes of the quotient algebras of this type:

$\left.e_{1}\right){ }^{\epsilon} \ell_{p, q-1}\left({ }^{\epsilon} C_{q, p-1}\right)$ with $\mathcal{A} \rightarrow \widetilde{\mathcal{A}}, \mathcal{A} \rightarrow \overline{\mathcal{A}}, \mathcal{A} \rightarrow \overline{\widetilde{\mathcal{A}}}, p-q \equiv 1(\bmod 8), q \equiv 0$ $(\bmod 2)$.

$\left.e_{2}\right){ }^{\epsilon} C \ell_{p, q-1}\left({ }^{\epsilon} C \ell_{q, p-1}\right)$ with $\mathcal{A} \rightarrow \widetilde{\mathcal{A}}, \mathcal{A} \rightarrow \overline{\mathcal{A}^{\star}}, \mathcal{A} \rightarrow \overline{\widetilde{\mathcal{A}^{\star}}}, p-q \equiv 1(\bmod 8)$, $q \equiv 1(\bmod 2)$. 
2) The quotient algebras ${ }^{\epsilon} C \ell_{p, q-1}\left({ }^{\epsilon} C \ell_{q, p-1}\right), p-q \equiv 5(\bmod 8)$.

In this case the initial algebra $C \ell_{p, q}$ has the double quaternionic division ring $\mathbb{K} \simeq \mathbb{H} \oplus \mathbb{H}$ and its subalgebras $C \ell_{p, q-1}$ and $C \ell_{q, p-1}$ are of the type $p-q \equiv 4$ $(\bmod 8)$ or $p-q \equiv 6(\bmod 8)$ with the $\operatorname{ring} \mathbb{K} \simeq \mathbb{H}$. Therefore, in this case the pseudoautomorphism $\mathcal{A} \rightarrow \overline{\mathcal{A}}$ is equivalent to the particle-antiparticle conjugation $C^{\prime}$. As in the previous case the antiautomorphism $\mathcal{A} \rightarrow \widetilde{\mathcal{A}}$ is transferred at any $p-q \equiv 5(\bmod 8)$. For this type in dependence on the number $q$ there are two different classes:

$\left.f_{1}\right){ }^{\epsilon} C_{p, q-1}\left({ }^{\epsilon} C \ell_{q, p-1}\right)$ with $\mathcal{A} \rightarrow \widetilde{\mathcal{A}}, \mathcal{A} \rightarrow \overline{\mathcal{A}}, \mathcal{A} \rightarrow \overline{\widetilde{\mathcal{A}}}, p-q \equiv 5(\bmod 8), q \equiv 0$ $(\bmod 2)$.

$\left.f_{2}\right){ }^{\epsilon} \ell_{p, q-1}\left({ }^{\epsilon} C \ell_{q, p-1}\right)$ with $\mathcal{A} \rightarrow \widetilde{\mathcal{A}}, \mathcal{A} \rightarrow \overline{\mathcal{A}^{\star}}, \mathcal{A} \rightarrow \overline{\widetilde{\mathcal{A}^{\star}}}, p-q \equiv 5(\bmod 8)$, $q \equiv 1(\bmod 2)$.

In accordance with Theorem 14, in the case of odd-dimensional spaces $\mathbb{R}^{p, q}$ and $\mathbb{C}^{p+q}$ the algebra homomorphisms $C \ell_{p, q} \rightarrow C \ell_{p, q-1}, C \ell_{p, q} \rightarrow \ell_{q, p-1}$ and $\mathbb{C}_{p+q} \rightarrow \mathbb{C}_{p+q-1}$ induce group homomorphisms $\operatorname{Pin}(p, q) \rightarrow \operatorname{Pin}(p, q-$ $1), \operatorname{Pin}(p, q) \rightarrow \operatorname{Pin}(q, p-1), \operatorname{Pin}(p+q, \mathbb{C}) \rightarrow \operatorname{Pin}(p+q-1, \mathbb{C})$.

Theorem 15. 1) If $\mathbb{F}=\mathbb{C}$ and $\mathbf{P i n}^{a, b, c, d, e, f g}(n+1, \mathbb{C}) \simeq \operatorname{Pin}^{a, b, c, d, e, f, g}(n, \mathbb{C}) \cup$ $\operatorname{Pin}^{a, b, c, d, e, f, g}(n, \mathbb{C})$ are universal coverings of the complex orthogonal groups $O(n+1, \mathbb{C})$, then in the result of the homomorphic mapping $\epsilon: \operatorname{Pin}(n+1, \mathbb{C}) \rightarrow$ $\operatorname{Pin}(n, \mathbb{C})$ we have the following quotient groups:

$$
\begin{aligned}
\operatorname{Pin}^{b}(n, \mathbb{C}) & \simeq \frac{\left(\operatorname{Spin}_{+}(n, \mathbb{C}) \odot \mathbb{Z}_{2} \otimes \mathbb{Z}_{2}\right)}{\mathbb{Z}_{2}} \\
\operatorname{Pin}^{b, d}(n, \mathbb{C}) & \\
\operatorname{Pin}^{b, e, g}(n, \mathbb{C}) & \simeq \frac{\left(\operatorname{Spin}_{+}(n, \mathbb{C}) \odot C^{b, e, g}\right)}{\mathbb{Z}_{2}}
\end{aligned}
$$

if $n+1 \equiv 1(\bmod 4)$ and

$$
\begin{aligned}
\operatorname{Pin}^{c, d, g}(n, \mathbb{C}) & \simeq \frac{\left(\operatorname{Spin}_{+}(n, \mathbb{C}) \odot C^{c, d, g}\right)}{\mathbb{Z}_{2}}, \\
\operatorname{Pin}^{a, b, c}(n, \mathbb{C}) & \simeq \frac{\left(\operatorname{Spin}_{+}(n, \mathbb{C}) \odot C^{a, b, c}\right)}{\mathbb{Z}_{2}} \\
\operatorname{Pin}^{c, e, f}(n, \mathbb{C}) & \simeq \frac{\left(\operatorname{Spin}_{+}(n, \mathbb{C}) \odot C^{c, e, f}\right)}{\mathbb{Z}_{2}}
\end{aligned}
$$


if $n+1 \equiv 3(\bmod 4)$.

2) If $\mathbb{F}=\mathbb{R}$ and $\operatorname{Pin}^{a, b, c, d, e, f, g}(p, q) \simeq \operatorname{Pin}^{a, b, c, d, e, f, g}(p, q-1) \cup$ $\omega \operatorname{Pin}^{a, b, c, d, e, f, g}(p, q-1), \operatorname{Pin}^{a, b, c, d, e, f, g}(p, q) \simeq \operatorname{Pin}^{a, b, c, d, e, f, g}(q, p-1) \cup$

$\omega \mathbf{P i n}^{a, b, c, d, e, f, g}(q, p-1)$ are universal coverings of the orthogonal groups $O(p, q)$ then in the results of the homomorphic mappings $\epsilon: \operatorname{Pin}(p, q) \rightarrow \operatorname{Pin}(p, q-1)$ and $\epsilon: \operatorname{Pin}(p, q) \rightarrow \operatorname{Pin}(q, p-1)$ we have the following quotient groups:

$$
\begin{aligned}
\operatorname{Pin}^{b}(p, q-1) & \simeq \frac{\left(\operatorname{Spin}_{+}(p, q-1) \odot \mathbb{Z}_{2} \otimes \mathbb{Z}_{2}\right)}{\mathbb{Z}_{2}} \\
\boldsymbol{P i n}^{b}(q, p-1) & \simeq \frac{\left(\operatorname{Spin}_{+}(q, p-1) \odot \mathbb{Z}_{2} \otimes \mathbb{Z}_{2}\right)}{\mathbb{Z}_{2}} \\
\operatorname{Pin}^{a, b, c}(p, q-1) & \simeq \frac{\left(\operatorname{Spin}_{+}(p, q-1) \odot C^{a, b, c}\right)}{\mathbb{Z}_{2}} \\
\operatorname{Pin}^{a, b, c}(q, p-1) & \simeq \frac{\left(\operatorname{Spin}_{+}(q, p-1) \odot C^{a, b, c}\right)}{\mathbb{Z}_{2}}
\end{aligned}
$$

if $p-q \equiv 1(\bmod 8)$ and

$$
\begin{aligned}
\operatorname{Pin}^{b, d, f}(p, q-1) & \simeq \frac{\left(\operatorname{Spin}_{+}(p, q-1) \odot C^{b, d, f}\right)}{\mathbb{Z}_{2}} \\
\operatorname{Pin}^{b, d, f}(q, p-1) & \simeq \frac{\left(\operatorname{Spin}_{+}(q, p-1) \odot C^{b, d, f}\right)}{\mathbb{Z}_{2}} \\
\operatorname{Pin}^{b, e, g}(p, q-1) & \simeq \frac{\left(\operatorname{Spin}_{+}(p, q-1) \odot C^{b, e, g}\right)}{\mathbb{Z}_{2}} \\
\operatorname{Pin}^{b, e, g}(q, p-1) & \simeq \frac{\left(\operatorname{Spin}_{+}(q, p-1) \odot C^{b, e, g}\right)}{\mathbb{Z}_{2}}
\end{aligned}
$$

if $p-q \equiv 5(\bmod 8)$.

Proof. As it has been shown in Theorem 14, over the field $\mathbb{F}=\mathbb{C}$ for the case $n+1 \equiv 1(\bmod 4)$ the antiautomorphism $\mathcal{A} \rightarrow \widetilde{\mathcal{A}}$ and pseudoautomorphism $\mathcal{A} \rightarrow \overline{\mathcal{A}}$ are transferred from $\mathbb{C}_{n+1}$ to $\mathbb{C}_{n}$ if the real subalgebra $C \ell_{p, q} \subset \mathbb{C}_{n+1}$ possesses the double rings $\mathbb{K} \simeq \mathbb{R} \oplus \mathbb{R}, \mathbb{K} \simeq \mathbb{H} \oplus \mathbb{H}, p-q \equiv 1,5(\bmod 8)$. Further, the quotient algebra ${ }^{\epsilon} \mathbb{C}_{n}$ generates the quotient group $\operatorname{Pin}^{\epsilon}(n, \mathbb{C})$. In the case of $\mathbb{K} \simeq \mathbb{R} \oplus \mathbb{R}$ we have $\operatorname{Pin}^{b}(n, \mathbb{C})$, since the pseudoautomorphism $\mathcal{A} \rightarrow \overline{\mathcal{A}}$ is equivalent to the identical transformations for $\mathbb{K} \simeq \mathbb{R} \oplus \mathbb{R}$. The automorphism group of ${ }^{\epsilon} \mathbb{C}_{n}$ is $\operatorname{Aut}\left({ }^{\epsilon} \mathbb{C}_{n}\right)=\{\mathrm{Id}, \sim \mathcal{\sim}\}\{1, T\}$ with the multiplication 
table

\begin{tabular}{|c||c|c|}
\hline & 1 & $T$ \\
\hline \hline 1 & 1 & $T$ \\
\hline$T$ & $T$ & 1 \\
\hline
\end{tabular}$\sim$\begin{tabular}{|c||c|c|}
\hline & Id & $\sim$ \\
\hline \hline Id & Id & $\sim$ \\
\hline$\sim$ & $\sim$ & Id \\
\hline
\end{tabular}

It is easy to see that $\{1, T\} \sim\{\mathrm{Id}, \sim\} \simeq\{\mathrm{I}, \mathrm{E}\} \simeq \mathbb{Z}_{2}$. Therefore, the double covering $C^{b}$ of the reflection group is isomorphic to $\mathbb{Z}_{2} \otimes \mathbb{Z}_{2}$.

In the case of $\mathbb{K} \simeq \mathbb{H} \oplus \mathbb{H}$ we have the quotient group $\operatorname{Pin}^{b, d}(n, \mathbb{C})$. At this point, a set of the discrete transformations of the space $\mathbb{C}^{n}$ associated with $\epsilon \mathbb{C}_{n}$ is defined by a three-element set $\{1, T, C\} \sim\{\mathrm{I}, \mathrm{E}, \Pi\}$, where $\{\mathrm{I}, \mathrm{E}, \Pi\}$ is an automorphism set of $\epsilon_{\mathbb{C}_{n}}, \mathrm{E}$ and $\Pi$ are the matrices of $\mathcal{A} \rightarrow \widetilde{\mathcal{A}}$ and $\mathcal{A} \rightarrow \overline{\mathcal{A}}$, respectively. It is easy to see that the set $\{1, T, C\} \sim\{\mathrm{I}, \mathrm{E}, \Pi\}$ does not form a group.

Further, when the subalgebra $C_{p, q} \subset \mathbb{C}_{n+1}$ has the complex ring $\mathbb{K} \simeq \mathbb{C}$ $(p-q \equiv 3,7(\bmod 8))$, the transformations $\mathcal{A} \rightarrow \widetilde{\mathcal{A}}, \mathcal{A} \rightarrow \overline{\mathcal{A}^{\star}}$ and $\mathcal{A} \rightarrow \overline{\widetilde{\mathcal{A}^{\star}}}$ are transferred from $\mathbb{C}_{n+1}$ to $\mathbb{C}_{n}, n+1 \equiv 1(\bmod 4)$. In this case we have the quotient group $\operatorname{Pin}^{\epsilon}(n, \mathbb{C}) \sim \operatorname{Pin}^{b, e, g}(n, \mathbb{C})$. The automorphism group of $\epsilon \mathbb{C}_{n}$ is $\operatorname{Aut}\left({ }^{\epsilon} \mathbb{C}_{n}\right)=\{\mathrm{Id}, \stackrel{\sim}{ }, \bar{\star}, \overline{\widetilde{\star}}\} \sim\{1, T, C P, C P T\}$ with the multiplication table

\begin{tabular}{|c|c|c|c|c|c|c|c|c|c|c|}
\hline & 1 & $T$ & $C P$ & $C P T$ & \multirow{5}{*}{$\sim$} & & Id & $\sim$ & $\bar{\star}$ & $\overline{\widetilde{\star}}$ \\
\hline 1 & 1 & $T$ & $C P$ & $C P T$ & & $\overline{\mathrm{Id}}$ & Id & $\sim$ & $\bar{\star}$ & $\overline{\widetilde{\star}}$ \\
\hline$T$ & $T$ & 1 & $C P T$ & $C P$ & & $\sim$ & $\sim$ & Id & $\overline{\widetilde{\star}}$ & $\bar{\star}$ \\
\hline$C P$ & $C P$ & $C P T$ & 1 & $T$ & & $\bar{\star}$ & $\mp$ & $\overline{\widetilde{\star}}$ & Id & $\sim$ \\
\hline$C P T$ & $C P T$ & $C P$ & $T$ & 1 & & $\overline{\widetilde{\star}}$ & $\overline{\widetilde{\star}}$ & $\bar{\star}$ & $\sim$ & Id \\
\hline
\end{tabular}

Therefore, $\operatorname{Aut}\left({ }^{\epsilon} \mathbb{C}_{n}\right) \simeq \mathbb{Z}_{2} \otimes \mathbb{Z}_{2}$, and we see that $\operatorname{Aut}\left(\epsilon^{\epsilon} \mathbb{C}_{n}\right)=$ $\{\mathrm{Id}, \sim, \bar{\star}, \overline{\widetilde{\star}}\}$ is a subgroup of the extended automorphism $\operatorname{group} \operatorname{Ext}\left(\mathbb{C}_{n}\right)=$ $\{\mathrm{Id}, \star, \sim \widetilde{\star},-\bar{\star}, \widetilde{\sim}, \overline{\widetilde{\star}}\}$ considered in the section 3. When $T^{2}=(C P)^{2}=$ $(C P T)^{2}= \pm 1$ and $T(C P)=-(C P) T$ we have a group $\operatorname{Aut}\left({ }^{\epsilon} \mathbb{C}_{n}\right)=$ $\{\mathrm{I}, \mathrm{E}, \mathrm{K}, \mathrm{F}\} \subset \operatorname{Ext}\left(\mathbb{C}_{n}\right)$, where $\operatorname{Ext}\left(\mathbb{C}_{n}\right)=\{\mathrm{I}, \mathrm{W}, \mathrm{E}, \mathrm{C}, \Pi, \mathrm{K}, \mathrm{S}, \mathrm{F}\}$. Therefore, $\operatorname{Pin}^{b, e, g}(n, \mathbb{C}) \simeq\left(\operatorname{Spin}_{+}(n, \mathbb{C}) \odot C^{b, e, g}\right) / \mathbb{Z}_{2}$, where $C^{b, e, g}$ are the four double coverings of the Gauss-Klein viergruppe $\mathbb{Z}_{2} \otimes \mathbb{Z}_{2}$; that is, $C^{b, e, g}$ are the groups $\mathbb{Z}_{2} \otimes \mathbb{Z}_{2} \otimes \mathbb{Z}_{2}$ (when $b=e=g=+$ ), $D_{4}$ (dihedral group, when there are two pluses and one minus in the triple $b, e, g$ ), $\mathbb{Z}_{4} \otimes \mathbb{Z}_{2}$ (when there are two minuses and one plus in $b, e, g$ ), and $Q_{4}$ (quaternions, when $b=e=g=-$ ). Since Aut $\left({ }^{\epsilon} \mathbb{C}_{n}\right)=\{\mathrm{I}, \mathrm{E}, \mathrm{K}, \mathrm{F}\}$ is a subgroup of $\operatorname{Ext}\left(\mathbb{C}_{n}\right)$, then we can define a 
structure of Aut $\left({ }^{\epsilon} \mathbb{C}_{n}\right)$ using Theorem 6. First of all, we see that there are four different types of $\operatorname{Aut}\left({ }^{\epsilon} \mathbb{C}_{n}\right)$ :

$$
\begin{aligned}
& \operatorname{Aut}^{1}\left({ }^{\epsilon} \mathbb{C}_{n}\right)=\left\{I, \mathcal{E}_{j_{1} j_{2} \cdots j_{k}}, \mathcal{E}_{\beta_{1} \beta_{2} \cdots \beta_{b}}, \mathcal{E}_{d_{1} d_{2} \cdots d_{g}}\right\}, \\
& \operatorname{Aut}^{2}\left({ }^{\epsilon} \mathbb{C}_{n}\right)=\left\{I, \mathcal{E}_{j_{1} j_{2} \cdots j_{k}}, \mathcal{E}_{\alpha_{1} \alpha_{2} \cdots \alpha_{a}}, \mathcal{E}_{c_{1} c_{2} \cdots c_{s}}\right\}, \\
& \operatorname{Aut}^{3}\left({ }^{\epsilon} \mathbb{C}_{n}\right)=\left\{I, \mathcal{E}_{i_{1} i_{2} \cdots i_{p+q-k}}, \mathcal{E}_{\beta_{1} \beta_{2} \cdots \beta_{b}}, \mathcal{E}_{c_{1} c_{2} \cdots c_{s}}\right\}, \\
& \operatorname{Aut}^{4}\left({ }^{\epsilon} \mathbb{C}_{n}\right)=\left\{I, \mathcal{E}_{i_{1} i_{2} \cdots i_{p+q-k}}, \mathcal{E}_{\alpha_{1} \alpha_{2} \cdots \alpha_{a}}, \mathcal{E}_{d_{1} d_{2} \cdots d_{g}}\right\} .
\end{aligned}
$$

All the elements $\mathrm{E}=\mathcal{E}_{j_{1} j_{2} \cdots i_{k}}, \mathrm{~K}=\mathcal{E}_{\beta_{1} \beta_{2} \cdots \beta_{b}}, \mathrm{~F}=\mathcal{E}_{d_{1} d_{2} \cdots d_{g}}$ of $\operatorname{Aut}^{1}\left({ }^{\epsilon} \mathbb{C}_{n}\right)$ are even products, that is, $k \equiv 0(\bmod 2), b \equiv 0(\bmod 2)$ and $g \equiv 0(\bmod 2)$. The element $\mathrm{K}$ commutes with $\mathrm{F}$ at $u \equiv 0(\bmod 2)$ and commutes with $\mathrm{E}$ at $u(m+v) \equiv 0(\bmod 2)$. In turn, from (88) $)$ it follows that $\mathrm{F}$ commutes with $\mathrm{E}$ at $m(v+u) \equiv 0(\bmod 2)$. In this case we have an Abelian group Aut $\left({ }^{\epsilon} \mathbb{C}_{n}\right) \simeq$ $\mathbb{Z}_{2} \otimes \mathbb{Z}_{2}$ if the elements $\mathrm{E}, \mathrm{K}, \mathrm{F}$ have positive squares $(b=e=g=+)$. And also we have the groups Aut $\left({ }^{\epsilon} \mathbb{C}_{n}\right) \simeq \mathbb{Z}_{4}$ with the signatures $(b, e, g)$, where among $b, e, g$ there are two minuses and one plus. Otherwise, we have non-Abelian groups $Q_{4} / \mathbb{Z}_{2}$ and $D_{4} / \mathbb{Z}_{2}$. In the case of Aut ${ }^{2}\left({ }^{\epsilon} \mathbb{C}_{n}\right)$ the elements $\mathrm{E}, \mathrm{K}, \mathrm{F}$ are both even and odd: $k \equiv 0(\bmod 2), a \equiv 1(\bmod 2), s \equiv 1(\bmod 2)$. The element $\mathrm{K}=\mathcal{E}_{\alpha_{1} \alpha_{2} \cdots \alpha_{a}}$ commutes with $\mathrm{F}=\mathcal{E}_{c_{1} c_{2} \cdots c_{s}}$ at $m \equiv 0(\bmod 2)$ and commutes with $\mathrm{E}=\mathcal{E}_{j_{1} j_{2} \cdots j_{k}}$ at $m(u+l) \equiv 0(\bmod 2)$. In turn, from (87) it follows that $\mathrm{F}$ commutes with $\mathrm{E}$ at $u(l+m) \equiv 0(\bmod 2)$. At these conditions Aut ${ }^{2}\left({ }^{\epsilon} \mathbb{C}_{n}\right)$ coincides with the Abelian groups $\mathbb{Z}_{2} \otimes \mathbb{Z}_{2}$, otherwise we have the groups $Q_{4} / \mathbb{Z}_{2}, D_{4} / \mathbb{Z}_{2}$. It is easy to see that $\operatorname{Aut}^{3}\left({ }^{\epsilon} \mathbb{C}_{n}\right)$ and $\operatorname{Aut}^{4}\left({ }^{\epsilon} \mathbb{C}_{n}\right)$ have the analogous structure.

Further, when the subalgebra $C_{p, q} \subset \mathbb{C}_{n+1}$ has the complex ring $\mathbb{K} \simeq \mathbb{C}$ for $n+1 \equiv 3(\bmod 4)$, the transformations $\mathcal{A} \rightarrow \widetilde{\mathcal{A}^{\star}}, \mathcal{A} \rightarrow \overline{\mathcal{A}}$ and $\mathcal{A} \rightarrow \overline{\widetilde{\mathcal{A}^{\star}}}$ are transferred from $\mathbb{C}_{n+1}$ to $\mathbb{C}_{n}$. In this case we have the quotient group

\begin{tabular}{|c|c|c|c|c|c|c|c|c|c|c|}
\hline & 1 & $P T$ & $C$ & $C P T$ & \multirow{4}{*}{$\sim$} & & $\mathrm{Id}$ & $\tilde{\star}$ & - & $\overline{\widetilde{\star}}$ \\
\hline 1 & 1 & $P T$ & $C$ & $C P T$ & & Id & Id & $\widetilde{\star}$ & - & $\overline{\widetilde{\star}}$ \\
\hline$P T$ & $P T$ & 1 & $C P T$ & $C$ & & $\tilde{\star}$ & $\tilde{\star}$ & Id & $\overline{\widetilde{\star}}$ & - \\
\hline$C$ & $C$ & $C P T$ & 1 & $P T$ & & - & - & $\overline{\widetilde{\star}}$ & $\mathrm{Id}$ & $\tilde{\star}$ \\
\hline$C P T$ & $C P T$ & $C$ & $P T$ & 1 & & $\overline{\widetilde{\star}}$ & $\overline{\widetilde{\star}}$ & - & $\tilde{\star}$ & Id \\
\hline
\end{tabular}
$\operatorname{Pin}^{\epsilon}(n, \mathbb{C}) \sim \operatorname{Pin}^{c, d, g}(n, \mathbb{C})$. The automorphism group of the quotient algebra $\epsilon^{\epsilon}$ is $\operatorname{Aut}\left({ }^{\epsilon} \mathbb{C}_{n}\right)=\{\operatorname{Id}, \widetilde{\star},-\overline{\widetilde{\star}}\} \sim\{1, P T, C, C P T\}$ with the following multiplication table: 
From the table it follows that $\operatorname{Aut}\left(\epsilon_{\mathbb{C}_{n}}\right)=\{\operatorname{Id}, \widetilde{\star},-, \overline{\widetilde{\star}}\} \simeq \mathbb{Z}_{2} \otimes \mathbb{Z}_{2} \subset \operatorname{Ext}\left(\mathbb{C}_{n}\right)$. When $(P T)^{2}=C^{2}=(C P T)^{2}= \pm 1$ and $(P T) C=-C(P T)$ we have a group Aut $\left({ }^{\epsilon} \mathbb{C}_{n}\right)=\{\mathrm{I}, \mathrm{C}, \Pi, \mathrm{F}\} \subset \operatorname{Ext}\left(\mathbb{C}_{n}\right)$. Therefore, $\mathbf{P i n}^{c, d, g}(n, \mathbb{C}) \simeq\left(\operatorname{Spin}_{+}(n, \mathbb{C}) \odot\right.$ $\left.C^{c, d, g}\right) / \mathbb{Z}_{2}$, where $C^{c, d, g}$ are the four double coverings of $\mathbb{Z}_{2} \otimes \mathbb{Z}_{2}$. As in the previous case, since Aut $\left(\mathbb{C}_{n}\right)=\{\mathrm{I}, \mathrm{C}, \Pi, \mathrm{F}\}$ is the subgroup of $\operatorname{Ext}\left(\mathbb{C}_{n}\right)$, then we can define a group structure of Aut $\left({ }^{\epsilon} \mathbb{C}_{n}\right)$ using Theorem [. In this case we see that there are four different types of $\{\mathrm{I}, \mathrm{C}, \Pi, \mathrm{F}\}$ :

$$
\begin{aligned}
& \operatorname{Aut}^{1}\left({ }^{\epsilon} \mathbb{C}_{n}\right)=\left\{I, \mathcal{E}_{i_{1} i_{2} \cdots i_{p+q}-k}, \mathcal{E}_{\alpha_{1} \alpha_{2} \cdots \alpha_{a}}, \mathcal{E}_{d_{1} d_{2} \cdots d_{g}}\right\}, \\
& \operatorname{Aut}^{2}\left({ }^{\epsilon} \mathbb{C}_{n}\right)=\left\{I, \mathcal{E}_{i_{1} i_{2} \cdots i_{p+q-k}}, \mathcal{E}_{\beta_{1} \beta_{2} \cdots \beta_{b}}, \mathcal{E}_{c_{1} c_{2} \cdots c_{s}}\right\}, \\
& \operatorname{Aut}^{3}\left({ }^{\epsilon} \mathbb{C}_{n}\right)=\left\{I, \mathcal{E}_{j_{1} j_{2} \cdots j_{k}}, \mathcal{E}_{\alpha_{1} \alpha_{2} \cdots \alpha_{a}}, \mathcal{E}_{c_{1} c_{2} \cdots c_{s}}\right\}, \\
& \operatorname{Aut}^{4}\left({ }^{\epsilon} \mathbb{C}_{n}\right)=\left\{I, \mathcal{E}_{j_{1} j_{2} \cdots j_{k}}, \mathcal{E}_{\beta_{1} \beta_{2} \cdots \beta_{b}}, \mathcal{E}_{d_{1} d_{2} \cdots d_{g}}\right\} .
\end{aligned}
$$

In the group $\operatorname{Aut}^{1}\left({ }^{\epsilon} \mathbb{C}_{n}\right)$ the element $\Pi=\mathcal{E}_{\alpha_{1} \alpha_{2} \cdots \alpha_{a}}$ commutes with $\mathrm{F}=\mathcal{E}_{d_{1} d_{2} \cdots d_{g}}$ at $l \equiv 0(\bmod 2)($ see $(75))$ and also commutes with $C=\mathcal{E}_{i_{1} i_{2} \cdots i_{p+q-k}}$ at $l(m+$ $v) \equiv 0(\bmod 2)$. In turn, from $(89)$ it follows that $\mathrm{F} \in \operatorname{Aut}^{1}\left({ }^{\epsilon} \mathbb{C}_{n}\right)$ commutes with $C$ at $v(m+l) \equiv 0(\bmod 2)$. Therefore, at the conditions $l, l(m+v), v(m+$ $l) \equiv 0(\bmod 2)$ we have the groups $\mathbb{Z}_{2} \otimes \mathbb{Z}_{2}$ and $\mathbb{Z}_{4}$, otherwise Aut ${ }^{1}\left({ }^{\epsilon} \mathbb{C}_{n}\right)$ coincides with $Q_{4} / \mathbb{Z}_{2}, D_{4} / \mathbb{Z}_{2}$. It is easy to verify that $\operatorname{Aut}^{2}\left({ }^{\epsilon} \mathbb{C}_{n}\right)$, $\operatorname{Aut}^{3}\left({ }^{\epsilon} \mathbb{C}_{n}\right)$ and Aut $^{4}\left({ }^{\epsilon} \mathbb{C}_{n}\right)$ have the analogous structure.

When the subalgebra $C_{p, q} \subset \mathbb{C}_{n+1}$ has the double ring $\mathbb{K} \simeq \mathbb{R} \oplus \mathbb{R}$, where $p-q \equiv 1(\bmod 8)$ and $n+1 \equiv 3(\bmod 4)$, the transformations $\mathcal{A} \rightarrow \widetilde{\mathcal{A}^{\star}}, \mathcal{A} \rightarrow$ $\overline{\mathcal{A}^{\star}}$ and $\mathcal{A} \rightarrow \overline{\widetilde{\mathcal{A}}}$ are transferred from $\mathbb{C}_{n+1}$ to $\mathbb{C}_{n}$. In accordance with Theorem 3 the pseudoautomorphism $\mathcal{A} \rightarrow \overline{\mathcal{A}}$ is reduced to the identical transformation in case of the real ring $\mathbb{K} \simeq \mathbb{R}$. Therefore, in this case the transformations $\mathcal{A} \rightarrow \overline{\mathcal{A}^{\star}}$ and $\mathcal{A} \rightarrow \overline{\widetilde{\mathcal{A}}}$ are reduced to $\mathcal{A} \rightarrow \mathcal{A}^{\star}$ and $\mathcal{A} \rightarrow \widetilde{\mathcal{A}}$, respectively. Thus,

\begin{tabular}{|c|c|c|c|c|c|c|c|c|c|c|}
\hline & 1 & $P$ & $T$ & $P T$ & \multirow{5}{*}{$\sim$} & & Id & $\star$ & $\sim$ & $\tilde{\star}$ \\
\hline 1 & 1 & $P$ & $T$ & $P T$ & & Id & Id & $\star$ & $\sim$ & $\tilde{\star}$ \\
\hline$P$ & $P$ & 1 & $P T$ & $T$ & & $\star$ & $\star$ & Id & $\tilde{\star}$ & $\sim$ \\
\hline$T$ & $T$ & $P T$ & 1 & $P$ & & $\sim$ & $\sim$ & $\tilde{\star}$ & Id & $\star$ \\
\hline$P T$ & $P T$ & $T$ & $P$ & 1 & & $\tilde{\star}$ & $\widetilde{\star}$ & $\sim$ & $\star$ & Id \\
\hline
\end{tabular}
we have the quotient group $\operatorname{Pin}^{\epsilon}(n, \mathbb{C}) \sim \operatorname{Pin}^{a, b, c}(n, \mathbb{C})$ and the automorphism group of $\epsilon^{\epsilon} \mathbb{C}_{n}$ is $\operatorname{Aut}\left({ }^{\epsilon} \mathbb{C}_{n}\right)=\{\mathrm{Id}, \star, \sim \widetilde{\sim}\} \sim\{1, P, T, P T\}$ (reflection group, see Proposition 11) with the multiplication table

The structure of $\operatorname{Aut}\left({ }^{\epsilon} \mathbb{C}_{n}\right)=\{\mathrm{Id}, \star, \sim, \widetilde{\star}\}=\{\mathrm{I}, \mathrm{W}, \mathrm{E}, \mathrm{C}\}$ is well studied (see Theorem [5). Thus, in this case we come again to the Dạbrowski groups 
$\operatorname{Pin}^{a, b, c}(n, \mathbb{C}) \simeq\left(\operatorname{Spin}_{+}(n, \mathbb{C}) \odot C^{a, b, c}\right) / \mathbb{Z}_{2}$, where $C^{a, b, c}$ are the four double coverings of $\mathbb{Z}_{2} \otimes \mathbb{Z}_{2}$ (see Theorem 111).

Further, when the subalgebra $C_{p, q} \subset \mathbb{C}_{n+1}$ has the double quaternionic ring $\mathbb{K} \simeq \mathbb{H} \oplus \mathbb{H}$, where $p-q \equiv 5(\bmod 8)$ and $n+1 \equiv 3(\bmod 4)$, the transformations $\mathcal{A} \rightarrow \widetilde{\mathcal{A}^{\star}}, \mathcal{A} \rightarrow \overline{\mathcal{A}^{\star}}$ and $\mathcal{A} \rightarrow \overline{\widetilde{\mathcal{A}}}$ are transferred from $\mathbb{C}_{n+1}$ to $\mathbb{C}_{n}$. In this case we have the quotient group $\operatorname{Pin}^{\epsilon}(n, \mathbb{C}) \sim \operatorname{Pin}^{c, e, f}(n, \mathbb{C})$. The automorphism group of the quotient algebra ${ }^{\epsilon} \mathbb{C}_{n}$ is $\operatorname{Aut}\left({ }^{\epsilon} \mathbb{C}_{n}\right)=\{\operatorname{Id}, \widetilde{\star}, \bar{\star}, \bar{\sim}\} \sim$ $\{1, P T, C P, C T\}$ with the following multiplication table:

\begin{tabular}{|c|c|c|c|c|c|c|c|c|c|c|}
\hline & 1 & $P T$ & $\overline{C P}$ & $C T$ & \multirow{5}{*}{$\sim$} & & Id & $\bar{\star}$ & $\bar{\star}$ & $\bar{\sim}$ \\
\hline 1 & 1 & $P T$ & $C P$ & $C T$ & & Id & Id & $\tilde{\star}$ & $\mp$ & $\approx$ \\
\hline$P T$ & $P T$ & 1 & $C T$ & $C P$ & & $\widetilde{\star}$ & $\widetilde{\star}$ & Id & $\bar{\sim}$ & $\bar{\nexists}$ \\
\hline$C P$ & $C P$ & $C T$ & 1 & $P T$ & & $\bar{\star}$ & $\bar{\star}$ & $\bar{\sim}$ & $\mathrm{Id}$ & $\widetilde{\star}$ \\
\hline$C T$ & $C T$ & $C P$ & $P T$ & 1 & & $\widetilde{\sim}$ & $\bar{\sim}$ & ॠ & $\widetilde{\star}$ & Id \\
\hline
\end{tabular}

From this table it follows that $\operatorname{Aut}\left({ }^{\epsilon} \mathbb{C}_{n}\right)=\{\operatorname{Id}, \widetilde{\star}, \bar{\star}, \bar{\sim}\} \simeq \mathbb{Z}_{2} \otimes \mathbb{Z}_{2} \subset \operatorname{Ext}\left(\mathbb{C}_{n}\right)$. When $(P T)^{2}=(C P)^{2}=(C T)^{2}= \pm 1$ and $(P T)(C T)=-(C P)(P T)$ we have a group Aut $\left({ }^{\epsilon} \mathbb{C}_{n}\right)=\{\mathrm{I}, \mathrm{C}, \mathrm{K}, \mathrm{S}\} \subset \operatorname{Ext}\left(\mathbb{C}_{n}\right)$. Therefore, $\operatorname{Pin}^{c, e, f}(n, \mathbb{C}) \simeq$ $\left(\operatorname{Spin}_{+}(n, \mathbb{C}) \odot C^{c, e, f}\right) / \mathbb{Z}_{2}$. Since Aut $\left({ }^{\epsilon} \mathbb{C}_{n}\right)=\{\mathrm{I}, \mathrm{C}, \mathrm{K}, \mathrm{S}\}$ is the subgroup of $\operatorname{Ext}\left(\mathbb{C}_{n}\right)$, then using Theorem $[$ we see that there are four different types of $\{\mathrm{I}, \mathrm{C}, \mathrm{K}, \mathrm{S}\}$ :

$$
\begin{aligned}
& \operatorname{Aut}^{1}\left({ }^{\epsilon} \mathbb{C}_{n}\right)=\left\{1, \mathcal{E}_{i_{1} i_{2} \cdots i_{p+q-k}}, \mathcal{E}_{\beta_{1} \beta_{2} \cdots \beta_{b}}, \mathcal{E}_{c_{1} c_{2} \cdots c_{s}}\right\}, \\
& \operatorname{Aut}^{2}\left({ }^{\epsilon} \mathbb{C}_{n}\right)=\left\{1, \mathcal{E}_{i_{1} i_{2} \cdots i_{p+q-k}}, \mathcal{E}_{\alpha_{1} \alpha_{2} \cdots \alpha_{a}}, \mathcal{E}_{d_{1} d_{2} \cdots d_{g}}\right\}, \\
& \left.\operatorname{Aut}^{3}{ }^{\epsilon} \mathbb{C}_{n}\right)=\left\{1, \mathcal{E}_{j_{1} j_{2} \cdots j_{k}}, \mathcal{E}_{\beta_{1} \beta_{2} \cdots \beta_{b}}, \mathcal{E}_{d_{1} d_{2} \cdots d_{g}}\right\}, \\
& \operatorname{Aut}^{4}\left({ }^{\epsilon} \mathbb{C}_{n}\right)=\left\{1, \mathcal{E}_{j_{1} j_{2} \cdots j_{k}}, \mathcal{E}_{\alpha_{1} \alpha_{2} \cdots \alpha_{a}}, \mathcal{E}_{c_{1} c_{2} \cdots c_{s}}\right\} .
\end{aligned}
$$

It is easy to see that a structure of $\operatorname{Aut}\left({ }^{\epsilon} \mathbb{C}_{n}\right)=\{I, C, K, S\}$ is analogous to the structure of other subgroups of $\operatorname{Ext}\left(\mathbb{C}_{n}\right)$ such as $\{I, E, K, F\},\{I, C, \Pi, F\}$ and $\{I, W, E, C\}$.

Let us consider now the quotient groups $\operatorname{Pin}^{\epsilon}(p, q-1), \operatorname{Pin}^{\epsilon}(q, p-1)$ over the field $\mathbb{F}=\mathbb{R}$. First of all, when the algebra $C \ell_{p, q}$ at $q \equiv 0(\bmod 2)$ has the double division $\operatorname{ring} \mathbb{K} \simeq \mathbb{R} \oplus \mathbb{R}, p-q \equiv 1(\bmod 8)$, the transformations $\mathcal{A} \rightarrow \widetilde{\mathcal{A}}, \mathcal{A} \rightarrow \overline{\mathcal{A}}$ and $\mathcal{A} \rightarrow \overline{\widetilde{\mathcal{A}}}$ are transferred from $C \ell_{p, q}$ to $C \ell_{p, q-1}$ or $C \ell_{q, p-1}$, where $C \ell_{p, q-1}$ and $C \ell_{q, p-1}$ are of the type $p-q \equiv 0(\bmod 8)$ or $p-q \equiv 2$ $(\bmod 8)$. However, in accordance with Theorem 3, the pseudoautomorphism 
$\mathcal{A} \rightarrow \overline{\mathcal{A}}$ is equivalent to Id for the ring $\mathbb{K} \simeq \mathbb{R}$. Therefore, the transformations $\mathcal{A} \rightarrow \overline{\mathcal{A}}$ and $\mathcal{A} \rightarrow \overline{\widetilde{\mathcal{A}}}$ are reduced to Id and $\mathcal{A} \rightarrow \widetilde{\mathcal{A}}$, respectively. For that reason an automorphism group of the quotient algebras ${ }^{\epsilon} C \ell_{p, q-1}$ and ${ }^{\epsilon} C \ell_{q, p-1}$ is equivalent to $\{\mathrm{Id}, \sim\} \simeq\{1, T\} \simeq \mathbb{Z}_{2}$ with the multiplication table (114). Thus, we have the quotient groups $\operatorname{Pin}^{b}(p, q-1) \simeq\left(\operatorname{Spin}_{+}(p, q-1) \odot \mathbb{Z}_{2} \otimes \mathbb{Z}_{2}\right) / \mathbb{Z}_{2}$ and $\operatorname{Pin}^{b}(q, p-1) \simeq\left(\operatorname{Spin}_{+}(q, p-1) \odot \mathbb{Z}_{2} \otimes \mathbb{Z}_{2}\right) / \mathbb{Z}_{2}$. In the case when $q \equiv 1(\bmod 2)$ the automorphisms $\mathcal{A} \rightarrow \widetilde{\mathcal{A}}, \mathcal{A} \rightarrow \overline{\mathcal{A}^{\star}}$ and $\mathcal{A} \rightarrow \overline{\widetilde{\mathcal{A}^{\star}}}$ are transferred from $C \ell_{p, q}$ to $C \ell_{p, q-1}$ or $C \ell_{q, p-1}$. Over the ring $\mathbb{K} \simeq \mathbb{R}$ the transformations $\mathcal{A} \rightarrow \frac{\mathcal{A}^{\star}}{\mathcal{A}^{\star}}$ and $\mathcal{A} \rightarrow \overline{\widetilde{\mathcal{A}^{\star}}}$ are reduced to $\mathcal{A} \rightarrow \mathcal{A}^{\star}$ and $\mathcal{A} \rightarrow \widetilde{\mathcal{A}^{\star}}$, respectively. Therefore, the automorphism groups of ${ }^{\epsilon} C_{p, q-1}\left({ }^{\epsilon} C l_{q, p-1}\right)$ is equivalent to $\left\{\mathrm{Id}, \star,^{\sim}, \widetilde{\star}\right\} \simeq$ $\{1, P, T, P T\}$ with the multiplication table (116). Thus, in this case we have the quotient groups $\left.\operatorname{Pin}^{a, b, c}(p, q-1) \simeq \operatorname{Spin}_{+}(p, q-1) \odot C^{a, b, c}\right) / \mathbb{Z}_{2}$ and $\operatorname{Pin}^{a, b, c}(q, p-1) \simeq\left(\operatorname{Spin}_{+}(q, p-1) \odot C^{a, b, c}\right) / \mathbb{Z}_{2}$, where $C^{a, b, c}$ are the four double coverings of the Gauss-Klein group $\mathbb{Z}_{2} \otimes \mathbb{Z}_{2}$.

Further, when the algebra $C_{p, q}$ at $q \equiv 0(\bmod 2)$ has the double quaternionic division ring $\mathbb{K} \simeq \mathbb{H} \oplus \mathbb{H}$, the automorphisms $\mathcal{A} \rightarrow \widetilde{\mathcal{A}}, \mathcal{A} \rightarrow \overline{\mathcal{A}}$ and $\mathcal{A} \rightarrow \overline{\widetilde{\mathcal{A}}}$ are transferred from $C_{p, q}$ to $C_{p, q-1}$ or $C_{q, p-1}$, where subalgebras $C \ell_{p, q-1}$ and $C \ell_{q, p-1}$ are of the type $p-q \equiv 4(\bmod 8)$ or $p-q \equiv 6(\bmod 8)$. In this case we have the quotient groups $\operatorname{Pin}^{\epsilon}(p, q-1) \sim \operatorname{Pin}^{b, d, f}(p, q-1)$ and $\operatorname{Pin}^{\epsilon}(q, p-1) \sim \operatorname{Pin}^{b, d, f}(q, p-1)$. The automorphism group of the quotient algebras ${ }^{\epsilon} C \ell_{p, q-1}$ and ${ }^{\epsilon} C \ell_{q, p-1}$ is $\left\{\mathrm{Id},{ }^{\sim},-, \bar{\sim}^{\sim}\right\} \sim\{1, T, C, C T\}$ with the following multiplication table:

\begin{tabular}{|c||c|c|c|c|}
\hline & 1 & $T$ & $C$ & $C T$ \\
\hline \hline 1 & 1 & $T$ & $C$ & $C T$ \\
\hline$T$ & $T$ & 1 & $C T$ & $C$ \\
\hline$C$ & $C$ & $C T$ & 1 & $T$ \\
\hline$C T$ & $C T$ & $C$ & $T$ & 1 \\
\hline \hline Id & Id & $\sim$ & - & $\sim$ \\
\hline$\sim$ & $\sim$ & Id & $\sim$ & - \\
\hline- & - & $\sim$ & Id & $\sim$ \\
\hline $\bar{\sim}$ & $\sim$ & - & $\sim$ & Id \\
\hline
\end{tabular}

From this table we see that $\{\mathrm{Id}, \sim,-, \bar{\sim}\} \simeq \mathbb{Z}_{2} \otimes \mathbb{Z}_{2} \subset \operatorname{Ext}\left(C \ell_{p, q}\right)$. When $T^{2}=C^{2}=(C T)^{2}= \pm 1$ and $T C=-C T$ we have a group $\{\mathrm{I}, \mathrm{E}, \Pi, \mathrm{S}\} \subset$ $\operatorname{Ext}\left(C_{p, q}\right)$. It is easy to see that a structure of $\{I, E, \Pi, S\}$ is analogous to the structure of other subgroups of Ext such as $\{\mathrm{I}, \mathrm{E}, \mathrm{K}, \mathrm{F}\},\{\mathrm{I}, \mathrm{C}, \Pi, \mathrm{F}\},\{\mathrm{I}, \mathrm{W}, \mathrm{E}, \mathrm{C}\}$ and $\{\mathrm{I}, \mathrm{C}, \mathrm{K}, \mathrm{S}\}$. Therefore, $\mathbf{P i n}^{b, d, f}(p, q-1) \simeq\left(\mathbf{S p i n}_{+}(p, q-1) \odot C^{b, d, f}\right) / \mathbb{Z}_{2}$ and $\mathbf{P i n}^{b, d, f}(q, p-1) \simeq\left(\mathbf{S p i n}_{+}(q, p-1) \odot C^{b, d, f}\right) / \mathbb{Z}_{2}$, where $C^{b, d, f}$ are the four double coverings of $\mathbb{Z}_{2} \otimes \mathbb{Z}_{2}$.

In the case when $q \equiv 1(\bmod 2)$ the automorphisms $\mathcal{A} \rightarrow \widetilde{\mathcal{A}}, \mathcal{A} \rightarrow \overline{\mathcal{A}^{\star}}$ 
and $\mathcal{A} \rightarrow \overline{\widetilde{\mathcal{A}^{\star}}}$ are transferred from $C \ell_{p, q}$ to $C \ell_{p, q-1}$ or $C \ell_{q, p-1}$. Therefore, the automorphism group of ${ }^{\epsilon} C \ell_{p, q-1}\left({ }^{\epsilon} C \ell_{q, p-1}\right)$ is equivalent to $\{\mathrm{Id}, \sim, \bar{\nexists}, \overline{\widetilde{\star}}\} \sim$ $\{1, T, C P, C P T\}$ with the multiplication table (115). Thus, in this case we have the quotient groups $\operatorname{Pin}^{b, e, g}(p, q-1) \simeq\left(\operatorname{Spin}_{+}(p, q-1) \odot C^{b, e, g}\right) / \mathbb{Z}_{2}$ and $\operatorname{Pin}^{b, e, g}(q, p-1) \simeq\left(\operatorname{Spin}_{+}(q, p-1) \odot C^{b, e, g}\right) / \mathbb{Z}_{2}$.

\section{Acknowledgements}

I am deeply grateful to Prof. J. S. R. Chisholm, to Prof. L. Dąbrowski and Prof. A. Trautman for sending me their interesting works.

\section{References}

[1] L. J. Alty, A. Chamblin, Spin Structures on Kleinian Manifolds, Class. Quantum Grav. 11, 2411-2415, (1994).

[2] L. J. Alty, A. Chamblin, Obstructions to Pin Structures on Kleinian Manifolds, J. Math. Phys. 37, 2001-2011, (1996).

[3] B. Ammann, Spin-Strukturen und das Spektrum des Dirac-Operators (Dissertation Freiburg 1998, Shaker-Verlag Aachen 1998).

[4] M. F. Atiyah, R. Bott, A. Shapiro, Clifford modules, Topology, 3, (Suppl. 1), 3-38, (1964).

[5] J. C. Baez, The Octonions, preprint math.RA/0105155 (2001).

[6] V. Bargmann, E. P. Wigner, Group theoretical discussion of relativistic wave equations, Proc. Nat. Acad. USA 34, 211-223 (1948).

[7] C. Bär, Das Spektrum von Dirac-Operatoren (Dissertation, Bonner Math. Schriften 217, 1991).

[8] H. Baum, Spin-Strukturen und Dirac-Operatoren über pseudoriemannschen Mannigfaltigkeiten (Teubner, Leipzig, 1981).

[9] V. B. Berestetskii, E. M. Lifshitz, L.P. Pitaevskii, Quantum Electrodynamics, Course of Theoretical Physics Vol. 4, 2nd Edition (Pergamon Press, Oxford, 1982).

[10] M. Berg, C. DeWitt-Morette, S. Gwo, E. Kramer, The Pin Groups in Physics: C, P, and T, preprint math-ph/0012006 (2000). 
[11] M. Blau, L. Dabrowski, Pin structures on manifolds quotiented by discrete groups, J. Geometry and Physics 6, 143-157, (1989).

[12] A. Borel, F. Hirzebruch, Characteristic classes and homogeneous spaces, Amer. J. Math. 80, 458-538, 81, 315-382, 82, 491-504 (1958, 1959, 1960).

[13] H. W. Braden, $N$-dimensional spinors: Their properties in terms of finite groups, J. Math. Phys. 26, 613-620 (1985).

[14] I. L. Buchbinder, D. M. Gitman, A. L. Shelepin, Discrete symmetries as automorphisms of proper Poincaré group, Int. J. Theor. Phys. 41, 753-790 (2002).

[15] P. Budinich, A. Trautman, The Spinorial Chessboard (Springer, Berlin, 1988).

[16] E. Cartan, Nombres complexes, in J. Molk, ed. : Encyclopédie des sciences mathématiques, Tome I, Vol.1, Fasc 4, art. 15, pp. 329-468 (1908).

[17] A. Chamblin, Some Applications of Differential Topology in General Relativity, J. Geometry and Physics 13, 357-377 (1994).

[18] A. Chamblin, On the Obstructions to Non-Cliffordian Pin Structures, Commun. Math. Phys. 164, 67-87, (1994).

[19] A. Chamblin, On the Superselection Sectors of Fermions, preprint DAMTP R-97/4; hep-th/9704099, (1997).

[20] C. Chevalley, The Algebraic Theory of Spinors (Columbia University Press, New York, 1954).

[21] C. Chevalley, The construction and study of certain important algebras, Publications of Mathematical Society of Japan No 1 (Herald Printing, Tokyo, 1955).

[22] J. S. R. Chisholm, R. S. Farwell, Properties of Clifford Algebras for Fundamental Particles, in Clifford (Geometric) Algebras, ed. W. Baylis (Birkhäuser, 1996), pp. 365-388.

[23] Y. Choquet-Bruhat, C. De Witt-Morette, M. Dillard-Bleick, Analysis, Manifolds and Physics (North-Holland Publ. Co., Amsterdam, 1982). 
[24] W. K. Clifford, Applications of Grassmann's extensive algebra, Amer. J. Math. 1, 350, (1878).

[25] W. K. Clifford, On the classification of geometric algebras, in R. Tucker, ed., Mathematical Papers by William Kingdon Clifford (Macmillan, London, 1982), pp. 397-401.

[26] J. F. Cornwell, Group Theory in Physics (Academic Press, San Diego, 1984).

[27] A. Crumeyrolle, Orthogonal and Symplectic Clifford Algebras, Spinor Structures (Kluwer Acad. Publ., Dordrecht, 1991).

[28] L. Dąbrowski, A. Trautman, Spinor structures on spheres and projective spaces, J. Math. Phys. 27, 2022-2088 (1986).

[29] L. Dąbrowski, R. Percacci, Diffeomorphisms, orientations, and pin structures in two dimensions, J. Math. Phys. 29, 580-593 (1987).

[30] L. Dąbrowski, Group Actions on Spinors (Bibliopolis, Naples, 1988).

[31] L. Dąbrowski, M. Rinaldi, Spin structures, spinors and the Dirac operator: real versus complex manifolds, J. Geometry and Physics 6, 651-656 (1989).

[32] B. De Witt, The Pin Groups in Physics, Phys. Rev. D41, p. 1901 (1990).

[33] C. De Witt-Morette, Shang-Jr Gwo, E. Kramer, Spin or Pin?, preprint (1997); http://www.rel.ph.utexas.edu/Members/cdewitt/SpinOrPin1.ps.

[34] V. L. Figueiredo, W. A. Rodrigues, Jr., E. C. Oliveira, Covariant, algebraic, and operator spinors, Int. J. Theor. Phys. 29, 371-395, (1990).

[35] Th. Friedrich, Dirac-Operatoren in der Riemannschen Geometrie (Vieweg-Verlag Braunschweig/Wiesbaden, 1997).

[36] Th. Friedrich, Cartan Spinor Bundles on Manifolds, preprint SFB 288, N 303 , TU-Berlin (1998).

[37] Th. Friedrich, A. Trautman, Clifford structures and spinor bundles, preprint SFB 288, N 251, TU-Berlin (1996).

[38] Th. Friedrich, A. Trautman, Spin spaces, Lipschitz groups, and spinor bundles, preprint SFB 288, N 362, TU-Berlin (1999). 
[39] I. M. Gel'fand, A. M. Yaglom, General relativistic-invariant equations and infinite-dimensional representations of the Lorentz group, $\mathrm{Zh}$. Ehksp. Teor. Fiz. 18, 703-733 (1948).

[40] I. M. Gel'fand, R. A. Minlos, Z. Ya. Shapiro, Representations of the Rotation and Lorentz Groups and their Applications (Pergamon Press, Oxford, 1963).

[41] R.P. Geroch, Spinor Structure of Spacetimes in General Relativity: I, J. Math. Phys. 9, 1739-1744 (1968).

[42] D. M. Gitman, A. L. Shelepin, Fields on the Poincaré Group: Arbitrary Spin Description and Relativistic Wave Equations, Int. J. Theor. Phys. 40(3), 603-684 (2001).

[43] H. Grassmann, Die Ausdehnungslehre (Berlin, 1862).

[44] A. Haefliger, Sur l'extension du groupe structural d'un espace fibre, C.R. Acad. Sci. Paris 243, 558-560 (1956).

[45] W. R. Hamilton, Lectures on Quaternions (Dublin, 1853).

[46] A. Hurwitz, Uber die Komposition der quadratischen Formen, Math. Ann. 88, 1-25 (1923).

[47] C. J. Isham, Spinor fields in four dimensional space-time, Proc. R. Soc. Lond. A. 364, 591-599 (1978).

[48] H. Joos, Zur darstellungstheorie der inhomogenen Lorentzgrouppe als grundlade quantenmechanische kinematick, Fortschr. Phys. 10, 65 (1962).

[49] M. Karoubi, Algebres de Clifford et K-Theory, Ann. Scient. Ec. Norm. Sup, $1^{e}$ serre, t.1, 161 (1968).

[50] M. Karoubi, K-Theory. An Introduction (Springer-Verlag, Berlin, 1979).

[51] R. C. Kirby, L. R. Taylor, Pin Structures on Low-Dimensional Manifolds, London Math. Soc. Lecture Notes, no. 151, CUP (1989).

[52] T. K. Kuo, Internal-symmetry groups and their automorphisms, Phys. Rev. D4, 3620-3637 (1971).

[53] T. D. Lee, G. C. Wick, Space inversion, time reversal, and other discrete symmetries, Phys. Rev. 148, 1385-1404 (1966). 
[54] R. Lipschitz, Untersuchungen über die Summen von Quadraten (Max Cohen und Sohn, Bonn, 1886).

[55] P. Lounesto, Clifford Algebras and Spinors (Cambridge University Press, Cambridge, 1997).

[56] H. B. Lowson, M.-L. Michelsohn, Spin Geometry (Princeton University Press, Princeton 1989).

[57] C. A. Manogue, J. Schray, Octonionic representations of Clifford algebras and triality, Found. Phys. 26, 17-70 (1996).

[58] L. Michel, Invariance in quantum mechanics and group extension, in Group Theoretical Concepts and Methods in Elementary Particle Physics, pages 135-200 (Gordon \& Breach, New York, 1964).

[59] J. Milnor, Spin structures on manifolds, Enseign. Math. 9, 198-203 (1963).

[60] H. Minkowski, Raum and Zeit, Phys. Zs. 10, 104 (1909).

[61] I. R. Porteous, Topological Geometry (van Nostrand, London, 1969).

[62] I. R. Porteous, Clifford Algebras and Classical Groups (Cambridge University Press, Cambridge, 1995).

[63] J. Radon, Lineare Scharen orthogonaler Matrizen, Abh. Math. Seminar Hamburg 1, 1-24 (1922).

[64] W. Rarita, J. Schwinger, On a theory of particles with half-integral spin, Phys. Rev. 60, 61 (1941).

[65] P. K. Rashevskii, The Theory of Spinors, (in Russian) Uspekhi Mat. Nauk 10, 3-110 (1955); English translation in Amer. Math. Soc. Transl. (Ser. 2) 6, 1 (1957).

[66] P. K. Rashevskii, About Mathematical Foundations of Quantum Electrodynamics, (in Russian) Uspekhi Mat. Nauk 13, 3-110 (1958).

[67] W. A. Rodrigues, Jr., V. L. Figueiredo, Real spin-Clifford bundle and the spinor structure of the spacetime, Int. J. Theor. Phys. 29, 413-424 (1990). 
[68] W. A. Rodrigues, Jr., E. C. Oliveira, Dirac and Maxwell equations in the Clifford and spin-Clifford bundles, Int. J. Theor. Phys. 29, 397-412 (1990).

[69] W. A. Rodrigues, Jr., Q. A. G. de Souza, The Clifford bundle and the nature of the gravitational field, Found. Phys. 23, 1465-1490 (1993).

[70] N. Salingaros, Realization, extension, and classification of certain physically important groups and algebras, J. Math. Phys. 22, 226-232, (1981).

[71] N. Salingaros, On the classification of Clifford algebras and their relation to spinors in $n$ dimensions, J. Math. Phys. 23(1), (1982).

[72] N. Salingaros, The relationship between finite groups and Clifford algebras, J. Math. Phys. 25, 738-742, (1984).

[73] R. Shaw, Finite geometry, Dirac groups and the table of real Clifford algebras, Univ. of Hull Maths Research Report, 7, no 1, (1994).

[74] Yu. M. Shirokov, Group theoretical analysis of the foundations of relativistic quantum mechanics. IV, V , Zh. Ehksp. Teor. Fiz. 34, 717-724 (1958); 36, 879-888 (1959).

[75] Yu. M. Shirokov, Spacial and time reflections in the relativistic theory, Zh. Ehksp. Teor. Fiz. 38, 140-150 (1960).

[76] Z. K. Silagadze, On the internal parity of antiparticles, Sov. J. Nucl. Phys. 55, 392-396 (1992).

[77] A. Trautman, Spinors and the Dirac operator on hypersurfaces. I. General Theory, J. Math. Phys. 33, 4011-4019, (1992).

[78] V. V. Varlamov, Fundamental Automorphisms of Clifford Algebras and an Extension of Dabrowski Pin Groups, Hadronic Journal 22, 497-535 (1999).

[79] V. V. Varlamov, Discrete Symmetries and Clifford Algebras, Int. J. Theor. Phys. 40, No. 4, 769-805 (2001).

[80] V. V. Varlamov, Clifford Algebras and Discrete Transformations of Spacetime, Proceedings of Third Siberian Conference on Mathematical Problems of Spacetime Physics of Complex Systems, Novosibirsk, June 20-22 2000. Publishing House of the Institute of Mathematics, Novosibirsk, pp. 97-135, 2001. 
[81] S. Weinberg, Feinman rules for any spin I \& II \& III, Phys. Rev. 133B, 1318-1332 \& 134B, 882-896 (1964) \& 181B, 1893-1899 (1969).

[82] G. S. Whiston, Compact Spinor Spacetimes, J. Phys. A: Math. Gen. 11, No. 7 (1978).

[83] E. Witt, Theorie der quadratischen Formen in beliebigen Körpern, J. Reine Angew. Math. 176, 31-44 (1937). 